\title{
SPATIAL POWER PROFILING METHOD USING VISUAL INFORMATION IN REACTORS WITH OPTICALLY TRANSPARENT COOLANTS
}

\author{
A Dissertation \\ by \\ JASON ALAN HEARNE \\ Submitted to the Office of Graduate and Professional Studies of \\ Texas A\&M University \\ in partial fulfillment of the requirements for the degree of \\ DOCTOR OF PHILOSOPHY
}

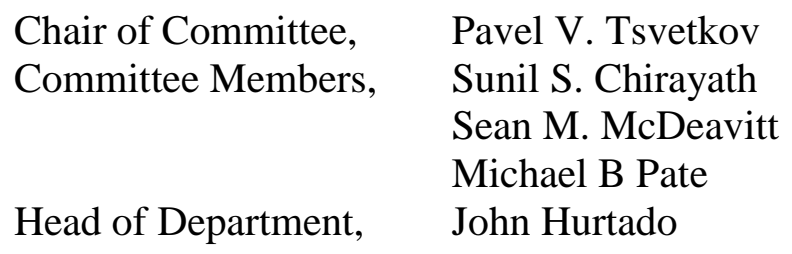

May 2019

Major Subject: Nuclear Engineering

Copyright 2019 Jason Hearne 


\begin{abstract}
The purpose of this dissertation is to develop a method of power profile reconstruction based upon visual instrumentation for nuclear reactors with optically transparent coolants. The detection of visible light produced by Cerenkov radiation at points outside of the active core region of a TRIGA reactor is correlated to the spatial power profile within the reactor. This is done by first using simple models in MCNP 6.1.1 beta to correlate the spatial and energy dependent electron flux within the coolant channels to the detectable Cerenkov radiation above the core by generating a response function. A detailed model of the whole core is used to tally the electron fluxes within the coolant channels, which then use the response function to produce a 2D map of the Cerenkov radiation observable above the core. An extension of the method using additional viewpoints that are at lateral offsets above the coolant channels is developed to allow for 3D information in the form of axial flux tilts to also be obtained. The potential use for this method is demonstrated by modelling various constant core power reactor perturbations and showing the ability of the power profile map to provide information about what change occurred. Various rod movements, coolant channel blockages and the placement of the TRIGA next to the graphite coupler box are all analyzed. Some additional analysis of the potential applicability of this method to different reactor designs is performed, with recommendations as to where and how it could be implemented.
\end{abstract}




\section{ACKNOWLEDGEMENTS}

I would like to thank my committee chair, Dr. Tsvetkov, and my committee

members, Dr. Chirayath, Dr. McDeavitt, and Dr. Pate, for their guidance and support throughout the course of this research.

Thanks also go to my friends and colleagues and the department faculty and staff for making my time at Texas A\&M University a great experience.

Finally, thanks to my mother and father for their encouragement and to my wife for her patience and love. 


\section{CONTRIBUTORS AND FUNDING SOURCES}

\section{Contributors}

This work was supervised by a dissertation committee consisting of Dr. Tsvetkov [advisor], Dr. Chirayath, and Dr. McDeavitt of the Department of Nuclear Engineering and Dr. Pate of the Department of Mechanical Engineering.

All other work conducted for the thesis (or) dissertation was completed by the student independently.

\section{Funding Sources}

This research is being performed using funding received from the DOE Office of Nuclear Energy's Nuclear Energy University Program, NEUP Award Number DENE0008306.

Because this dissertation was prepared as an account of work sponsored by an agency of the United States Government, neither the United States Government nor any agency thereof, nor any of their employees, makes any warranty, expressed or implied, or assumes any legal liability or responsibility for the accuracy, completeness, or usefulness of any information disclosed.

Any views, opinions, findings, conclusions, or recommendations expressed in this dissertation are those of the author and do not necessarily state or reflect the views of the Department of Energy Office of Nuclear Energy. 


\section{NOMENCLATURE}

TRIGA

NSC

SPND

MPFD

ICVD

DVCD

MCNP

FLiBe

PWR

TR

RR

VISED

BWR

FHR

HTGR
Training, Research, Isotopes, General Atomics: research reactor

Nuclear Science Center

Self-Powered Neutron Detector

Micro Pocket Fission Detector

Improved Cerenkov Viewing Device

Digital Cerenkov Viewing Device

Monte Carlo N-Particle: particle transport code

Lithium-Beryllium-Fluoride molten salt $\left(\mathrm{LiF}_{-} \mathrm{BeF}_{2}\right)$

Pressurized Water Reactor

Transient control Rod

Regulating Rod

Visual Editor: visualization program for MCNP

Boiling Water Reactor

Fluoride salt cooled High temperature Reactor

High Temperature Gas-cooled Reactor 


\section{TABLE OF CONTENTS}

Page

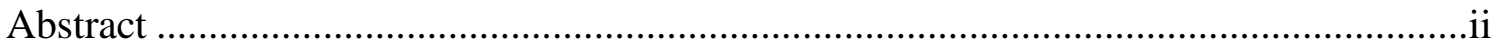

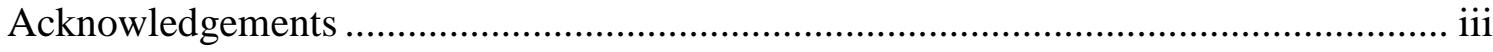

Contributors and funding sources ..............................................................................iv

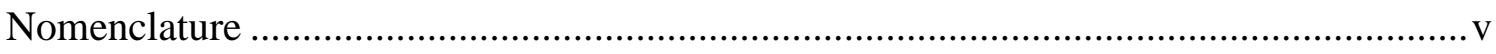

Table of Contents ....................................................................................................

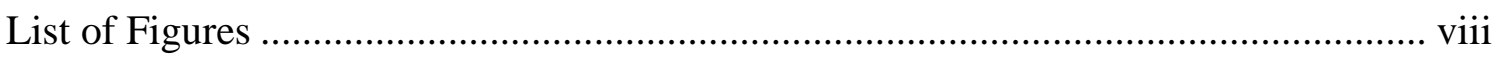

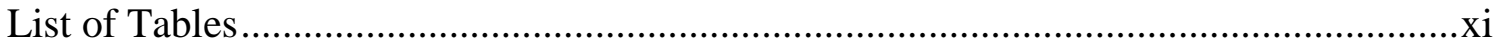

1. INTRODUCTION

1.1 Overview of reactor power instrumentation and current state of the art ..............1

1.2 Fundamental Physics of Cerenkov Radiation ..................................................

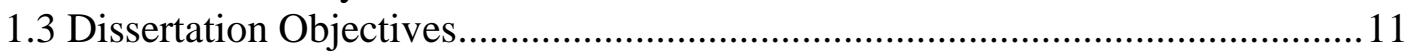

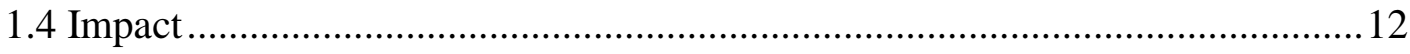

2. CERENKOV-BASED ANALYSIS METHODOLOGY …………….........................15

2.1 Production, transport and detection of Cerenkov radiation...................................15

2.2 Modelling Cerenkov in TRIGA ………………........................................ 19

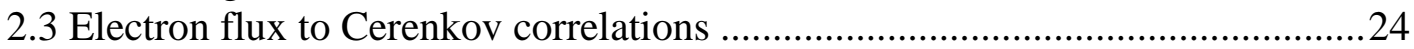

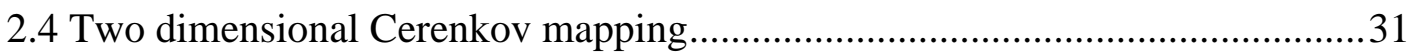

2.5 Reactor state change analysis with 2D Cerenkov maps ........................................36

3. THREE DIMENSIONAL CERENKOV PRODUCTION EXTRAPOLATION .........48

3.1 Use of multiple viewpoints and shadowing for 3D extrapolation.......................48

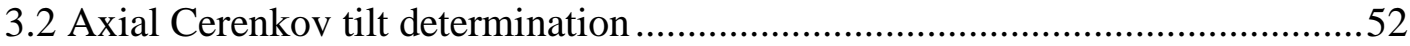

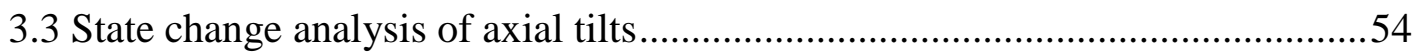

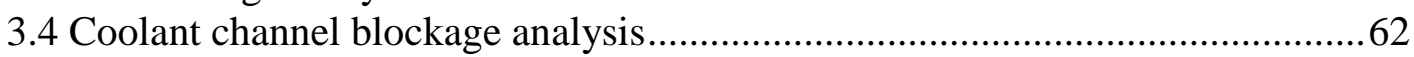

3.5 Example blockage detection and location ........................................................

4. GENERALIZATION OF SYSTEM FOR USE IN OTHER REACTOR TYPES ......75 


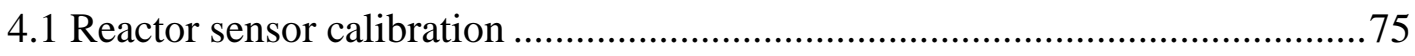

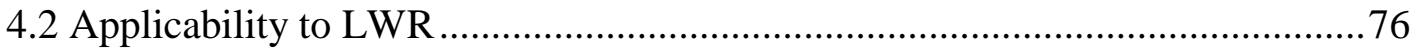

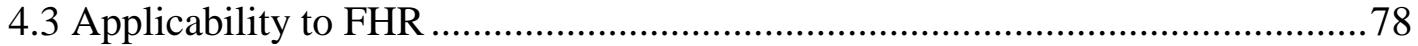

4.4 Applicability to HTGR or other gas cooled designs .................................... 79

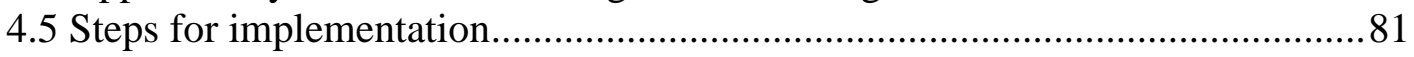

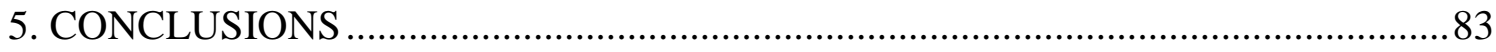

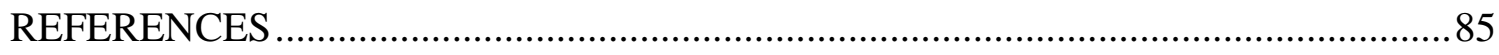

APPENDIX A ELECTRON TO CERENKOV FLUX CORRELATION FACTORS....95

APPENDIX B CERENKOV MODELING IN MCNP .......................................... 117

APPENDIX C SCRIPTS USED FOR POST PROCESSING AND PLOTTING .........123

vii 


\section{LIST OF FIGURES}

Figure 1 Diagram of Cerenkov production directions, with Cerenkov photons shown in blue [43]

Figure 2 Progression from neutrons in the fuel to Cerenkov detection above core

Figure 3 Axial (XY) cross section of the TRIGA core.

Figure 4 Vertical cross sections of row 4 (left) and row 6 (middle) and row 2 (right) displaying fuel pins, safety shims, the transient control rod, and the regulating rod.

Figure $5 \mathrm{XY}$ cross sections of 4 pin model showing pins, coolant and the reflecting boundaries. (Right) the red region is the portion of the channel containing the electron tallies.

Figure 6 Diagonal $\mathrm{X} / \mathrm{Y}$ vs $\mathrm{Z}$ cross section showing 2 of the pins and the cylindrical region of the channel being tallied. (Left) color corresponds to materials so that the coolant and fuel pins can be seen. (Right) the red region corresponds to one axial segment being tallied, while the yellow region contains the other 15 axial segments tallied in separate MCNP runs. The green region shows the bottom of the column of water above the channel where the Cerenkov detection plane is located, $2 \mathrm{~m}$ above the core's axial centerline

Figure $7 \mathrm{XY}$ cross sections of two versions of the 4 pin model, with the electron tally in the red region. (Left) has only 4 pins with a void around it, such that only gammas from those 4 pins can enter the channel. (Right) is reflected, such that the gammas reaching the coolant channel to produce electrons can come from an infinite lattice of pins.

Figure $8 \mathrm{XY}$ cross section of core showing coolant channels tallied for Cerenkov map and control rod positions. The red box corresponds to the region that is mapped in the subsequent power profiles.

Figure 9. This plot shows the Cerenkov estimates $2 \mathrm{~m}$ above the core, with and outline of the XY geometry of the core overlaying the image. The safety shims, transient rod (TR) and regulating rod (RR) positions are labeled. In this figure, the reactor is in the normal baseline critical state, with the regulating rod at $19.8 \mathrm{~cm}$, the shims at $28.4 \mathrm{~cm}$ and the transient rod fully withdrawn. This is used as a baseline to compare with other perturbed reactor states.

The scale of the plot is the normalized Cerenkov flux per fission neutron. ......33 
Figure 10 Two possible ways to measure the Cerenkov flux above the core. An array of photon detectors (left) or a single, multichannel detector or camera (right).

Figure 11 Absolute difference in Cerenkov above the core comparing the reactor with the regulating rod fully withdrawn to the baseline case with the regulating rod close to $50 \%$ withdrawal.

Figure 12 Relative difference in Cerenkov above the core comparing the reactor with the regulating rod fully withdrawn to the baseline case with the regulating rod close to $50 \%$ withdrawal

Figure 13 Absolute Cerenkov difference when inserting the transient rod to a position of $20 \mathrm{~cm}$.

Figure 14 Relative Cerenkov difference when inserting the transient rod to a position of $20 \mathrm{~cm}$

Figure 15 Reactor core when moved next to coupler box, showing XY (left) and YZ (right) views of the core

Figure 16 Map of total Cerenkov above core with graphite coupler adjacent to core. ....44

Figure 17 Absolute Cerenkov difference when moving the core next to the graphite coupler box.

Figure 18 Relative Cerenkov difference when moving the core near the coupler box. The white region at the top corresponds to a greater than $20 \%$ flux increase. . 46

Figure 19 Coolant channel segment contribution to Cerenkov observed at an offset from the channel centerline (left), where the shaded region is the only portion contributing to the signal at the offset location. Also, (right) the square coolant channel shadowing approximation, showing how the coolant channel and pins are approximated as square with an offset viewpoint perpendicular to the flat face of the square channel to simplify the geometry.

Figure 20 Contribution factors for the top, two middle and bottom segments in the coolant channel.

Figure 21 absolute tilt normalized to symmetric channel for each of the 81 coolant channels analyzed. The reactor is in the normal critical state.

Figure 22 Flux tilt map with regulating rod fully withdrawn. 
Figure 23 Change in flux tilt when removing the regulating rod and slightly inserting safety shims to maintain criticality.

Figure 24 Flux tilt in each channel when the transient rod is halfway inserted and the shims moved further out to maintain criticality.

Figure 25 Change in the flux tilt when inserting the transient rod to $20 \mathrm{~cm}$ and withdrawing the shims accordingly.

Figure 26 Relative change in the total Cerenkov flux directly above each channel when moving the coupler box next to the core and inserting shims to maintain criticality.

Figure 27 Change in flux tilt when moving the core next to the graphite coupler box....61

Figure 28 Total Cerenkov above core with channel 4,4 blocked 100\% at midplane.......64

Figure 29 Cerenkov difference above core with a blockage in channel 4,4 ...................65

Figure 30 Axial tilt map of core with a $100 \%$ blockage at the core midplane in channel 4,4. White space is where the tilt is outside of the previously used range. ......66

Figure 31 Rescaled plot of axial Cerenkov tilt with blocked channel. The large positive tilt in the affected channel stands out compared to the rest of the core.

Figure 32 Pin geometry and square channel approximation using four offset viewpoints, one in the direction of each neighboring pin.

Figure 33 Diagram demonstrating the effect that a partial block in different locations within the channel has on the offset viewpoints located on the same side and on the opposite side of the channel from the blockage.

Figure 34 Partial blockage at core midplane and its approximation for the model .........70 


\section{LIST OF TABLES}

Page

Table 1 Cerenkov detection in different viewpoints with $50 \%$ block in south side of channel at core midplane ........................................................................ 72

Table 2 Electron to Cerenkov flux correlations within a coolant channel .....................96 


\section{INTRODUCTION}

\subsection{Overview of reactor power instrumentation and current state of the art}

The goal of this dissertation is to develop a power profile reconstruction method that will enable the application of novel forms of instrumentation in reactors with optically transparent coolants to increase the diversity of instrumentation systems and to provide additional information about the reactor. This method aims to provide a way to characterize not only the total power of a reactor, but also the spatial power profile with a non-invasive system to enable higher resolution power mapping. To develop this instrumentation method, a system that uses visual detectors to measure Cerenkov fluxes in a TRIGA reactor is designed. Various different reactor states are modeled to obtain power profiles for the reactor using visual instrumentation to demonstrate the reconstruction method's ability to distinguish between different reactor states.

Currently reactor power instrumentation is primarily done using in core gamma or neutron detectors, or using temperature sensors [1] [2] [3]. Many instrumentation methods measure a small number of locations in or near the core to obtain the total core power. Some next generation reactor designs require improved power profile determination [4]. Higher precision spatial power profile measurements could also be used for code validation, xenon oscillation detection, or optimizing fuel loading and burnup.

Conventional temperature sensors use thermocouples to measure the temperature of the coolant in various positions in the reactor core or coolant loop [5] [6]. Temperature measurements are straightforward and have a long history of usage in many fields. In a steady state system, the coolant temperature rise across the reactor is directly proportional 
to the reactor's total power output. One disadvantage of many temperature sensors is that there can be a relatively slow response time to changes in the flux in the reactor. Temperature sensors can be placed within fuel elements to give a much faster response time, but this requires the presence of instrumentation within the actual fuel element, and is therefore usually only done in some research reactors [7] [8] [9]. More recently, some work has been done investigating the use of thermoacoustic sensors as a temperature sensing method that does not require external power [10] [11]. The use of fiber optic sensors for temperature measurement in reactors [12] and other industrial applications is also being pursued, both through conventional pyrometry, and through using temperature dependent transmission and reflection within the cables [13].

Neutron detectors function by detecting the products of a reaction between a neutron and material in the detector. This is often done by using an ionization chamber in which a neutron absorption causes ionization of a gas and the resulting signal is detected [1] [14] [15]. In fission chambers [16] [17], a fissile material is used as the neutron absorber; in many other ionization chamber designs, either natural or enriched boron is used, often in the lining of the chamber [18]. Compensated ion chambers have two ionization chambers, one with and one without the boron lining, so that the gamma count rate in the boron free chamber can be subtracted from the count rate in the absorber-lined chamber to more accurately estimate the number of pulses caused by neutron interactions by compensating for the gamma flux. Fission chambers have an advantage in that fission causes a very large deposition of energy, making it easier to discriminate between pulses caused by neutrons and those caused by gammas. Most conventional fission chambers are 
large, expensive to produce and can burn out after experiencing a large total neutron fluence. As the fissile content in the detectors is consumed, the signal output from the chamber will change [19] [20]. This limits the potential locations for placement and the total number of fission chambers that can be used in a reactor and thus the precision of the information that can be obtained about the spatial power profile.

Reactor power profile measurements can be made with self-powered neutron detectors (SPNDs) [21] [22]. These detectors are very small and have a sensitive emitter surrounded by an insulator and then a collector. The emitter absorbs a neutron to produce an electron, either promptly or with a delay through a short lived beta decay. The electron can then pass through the insulator to produce a negative charge in the collector. The creation of a negative charge in the collector and positive charge in the emitter creates a current between the two regions, which can then be detected. The system is self-powered because the emission of electrons by the emitter is the source of the current, and no external power supply is required, as would be in an ion chamber. Also, all portions are solid, making them more rugged and able to be used within the harsh environment in the core. The small size of SPND's allows multiple SPND's to be placed in a single small guide tube to obtain flux measurements at multiple axial locations along the tube, multiple assemblies can be instrumented to get a 3D power profile for the whole core.

A few different elements can be used in a SPND for the emitter with various advantages and disadvantages [23]. Rhodium is the most sensitive to neutrons due to its larger cross section, but it emits a beta as a delayed response, with a half-life of 44 seconds, also rhenium is more susceptible to burning out when placed in the core for a long time. 
Vanadium is similar to Rhodium except that it has a lower cross section resulting in lower sensitivity and slower burnup, and a longer delay in response with a decay half-life of 3.75 minutes. Silver as an emitter has an average sensitivity, burnup rate and a delayed signal. Cobalt has a prompt n-gamma interaction, but the gamma has a relatively low probability of producing a charge in the collector, resulting in a low sensitivity. Hafnium has a prompt n-gamma reaction with a larger cross section with cobalt, resulting in a higher though still low sensitivity and faster burnup. SPND's are less commonly used for direct reactor control due to the delays in the signal for most emitters [24]. Every choice of emitter has limitations, and there is a tradeoff between sensitivity, detector longevity and response speed.

A more recent development in neutron detection based power measurement is the use of Micro Pocket Fission Detectors (MPFDs) [25] [26] [27]. These detectors are much smaller than conventional fission chambers, with the diameter of the detector in the 1 to 3 mm range, allowing them to be inserted between fuel elements and to be bundled with a second cadmium coated MPFD for fast and thermal neutron flux measurements and a thermocouple at each detection node. The size of the detection gas pockets is very small, such that fission events in the detector only deposit a small portion (a few MeV) of the fission fragment's energy within the gas. This signal is still sufficient to be registered as a count and is much larger than background caused by gammas, because the gammainduced events have even smaller energy depositions within the tiny chamber [28]. The small size also allows multiple sets of detectors to be placed at different positions along a single instrumentation tube to obtain flux measurements in more locations. This newer 
technology has many potential benefits for obtaining detailed information about the flux profile within a reactor core. The direct detection of neutrons and ability to discriminate different neutron energies is an advantage. The requirement for the presence of instrumentation within the core is still a limitation, as well as the practical limits on the number of detectors that can be used.

Gamma detectors can either use ionization chambers to detect the ionization of the gas by gamma rays, solid-state semiconductor designs [29], or scintillation detectors [30]. Some in core gamma detector designs exist that use the production and detection of Cerenkov radiation within a detector gas to measure the high energy gamma flux [31] [32] . The gaseous Cerenkov tube detectors have the advantage of keeping all sensitive electronics further away from the core because the light detector is placed at the end of the tube, with the tube walls and gas being the only parts that are subjected to high radiation doses. Additionally, these designs can be tuned to discriminate between different gamma energies by selecting the pressure of the gas such that the required energy of recoil electrons for Cerenkov production is only present for interactions with gamma rays above the selected energy cutoff.

Neutrino detection is a less widely used method of remotely monitoring reactor power. This can be done by detecting the interactions of antineutrinos from the reactor with hydrogen gas in a large, external scintillation detector [33]. Neutrinos are almost completely unaffected by shielding, so this detector can be far removed from the core and potentially even the containment, with the limitation of the $1 / \mathrm{r}^{2}$ dependence of the antineutrino flux on the distance from the source. This detection method is sensitive to the 
portion of plutonium in the fuel, as $\mathrm{Pu}-239$ and U-235 produce different amounts of antineutrinos when fissioning. The sensitivity to plutonium content has potential applications in safeguards, but limits the usefulness as an operational power-monitoring channel due to the need to calibrate the detector to the reactor's current plutonium content. Neutrino detectors are also limited by the relatively high background from cosmic neutrinos and the fact that at a multi-unit power plant, all of the reactors would be contributing to the flux in a given detector.

A few systems that use Cerenkov radiation for power measurement have been designed [34] [35] [36] [37] [38] [39]. It has been shown that in a reactor operating in the intermediate to full power range, the Cerenkov fluxes are proportional to the total power. This has been used in the Tehran Research reactor to measure the total core power to provide another diverse and redundant power measurement system for reactor safety and operations [35]. Another type of Cerenkov based flux detector design involves using a small-bore metallic tube to transfer Cerenkov light produced in a small quartz crystal placed within the reactor to a detector [38]. This design uses the reflective, empty tube to efficiently transmit the light to the detector, but is limited by the fact that the tube must be present for each location measured. Additionally the high efficiency of light transfer is not necessary for Cerenkov detection in an operating reactor due to the large amount of light generated by the strong gamma fields.

Another similar existing technology is the use of Cerenkov detection in safeguards to verify the burnup of spent fuel assemblies. The ICVD and DCVD are used by the IAEA to verify the declared burnup of spent fuel assemblies and to ensure that fuel has not been 
diverted and replaced with non-fuel materials [40]. This method performs a $2 \mathrm{~d}$ spatial mapping of the Cerenkov flux generated by an assembly due to decay and therefore shares similarities with the goal of reactor power profiling. Data collection with the ICVD used in safeguards is generally done by hand in a spent fuel pool rather than by an automated system for an operating reactor. The ICVD is detecting the Cerenkov flux from a static system with gamma emission from radioactive decay as the ultimate source of the Cerenkov production, and the detection goal is the ability to determine whether or not a given assembly has the correct burnup and that no pins have been covertly removed or replaced. The ICVD has some issues with low light levels caused by the spent fuel not producing enough Cerenkov radiation for the detector. Problems with an insufficient light level are not present in an operating reactor, as the gamma field strength and thus Cerenkov production is much greater.

Visual instrumentation is commonly used for refueling and inspection during reactor outages [41]. An example of the ability to see Cerenkov radiation coming from decay gammas in coolant channels within a BWR during a refueling outage is publicly available in a demonstration of a refueling machine camera [42]. While an interesting demonstration of the transport of Cerenkov photons along a direct line of sight through a core's coolant channels, the conditions under which refueling occur are different than those present during operation. Offline reactor inspection is an established procedure and is not the focus of this research effort. 


\subsection{Fundamental Physics of Cerenkov Radiation}

The system considered here involves measuring Cerenkov radiation, so a brief summary of the physics involved is included. Cerenkov radiation occurs when a charged particle passes through a di-electric medium at a velocity greater than the phase velocity of light in that medium. The phase velocity of light in a medium is the speed of light divided by the frequency dependent refractive index in the medium as seen in equation 1 , where $n(\omega)$ is the index of refraction for the medium for light with a frequency of $\omega$. The frequency dependent phase velocity is thus given by

$$
c_{\text {phase }}(\omega)=\frac{c}{n(\omega)}
$$

Cerenkov radiation is emitted in a cone travelling outwards and in the direction of the particle, similar to the effect of a sonic boom produced by an object exceeding the speed of sound in air or some other sound propagating medium. The single wavelength wave front produced by a particle is shown by the blue arrows in Figure 1, where $\beta$ is the ratio of the particle's velocity to the speed of light, and $\theta$ is given by equation 2 :

$$
\theta(\lambda)=\cos \left(\frac{1}{n(\omega) \beta}\right)
$$




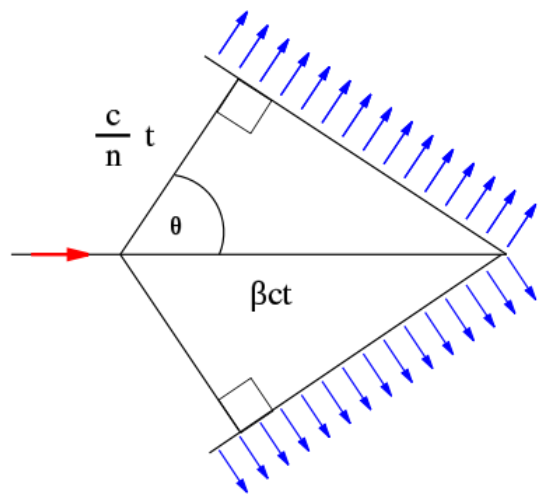

\section{Figure 1 Diagram of Cerenkov production directions, with Cerenkov photons shown in blue [43]}

In a real system, there is a diffraction effect where different wavelengths of light are emitted at different angles from the particle's path based upon their indices of refraction. The intensity of photons emitted in a wavelength is expressed as the energy emitted at a wavelength per unit path length traveled by the particle in the Frank-Tamm formula, given that $\beta$ is greater than $1 / \mathrm{n}(\omega)$. The Frank-Tamm formula can be seen in equation 3:

$$
\frac{d^{2} E}{d x d \omega}=\frac{q^{2}}{4 \pi} \mu(\omega) \omega\left(1-\frac{1}{\beta^{2} n^{2}(\omega)}\right)
$$

The emission rate is approximately proportional to the frequency, causing the intensity to peak in the upper blue and ultraviolet ranges for Cerenkov radiation produced in water, resulting in the distinctive blue glow that can be seen in operating reactors or beta emitting sources kept under water. Further into and beyond the ultraviolet range, the frequency dependent index of refraction of water drops below 1, preventing the production of Cerenkov photons with higher frequencies. 
The constraint that $\beta$ must be greater than $1 / \mathrm{n}$ also provides a lower limit for the velocity and energy of charged particles. Using an approximated constant $n$ of 1.33 for water, the minimum value of $\beta$ for Cerenkov production is .75 , which in the case of electrons corresponds to an energy of $261 \mathrm{keV}$, meaning that electrons with energies below $261 \mathrm{keV}$ will not produce Cerenkov radiation in water, and when an electron's energy drops below $261 \mathrm{keV}$, it stops giving off Cerenkov photons. However, slightly lower energy photons can still produce some Cerenkov radiation in water at shorter wavelengths, such as those in the near UV range, because the frequency dependent index of refraction goes up to a peak value of 1.44 , corresponding to a minimum value of $\beta$ of .694 , setting the cutoff electron energy at this limiting wavelength to $200 \mathrm{keV}$. So the true electron energy cutoff for Cerenkov production in water is $200 \mathrm{keV}$. This means that electrons with energies below $200 \mathrm{keV}$ do not need to be transported in the models as they will not produce any further Cerenkov photons.

The visible photons produced by Cerenkov radiation are transported through water with little attenuation. The attenuation of light in a medium is given by equation 4 :

$$
I=I_{0} e^{-\alpha x},
$$

where $\mathrm{I}$ is the intensity at a point, $\mathrm{I}_{0}$ is the intensity at the source and $\mathrm{x}$ is the distance from the source, and $\alpha$ is the absorption or attenuation coefficient of the medium. For a point source, the intensity is also multiplied by $1 / \mathrm{x}^{2}$ to account for spreading.

The attenuation coefficient in a medium, $\alpha$, is a function of the wavelength. In pure water, the attenuation coefficient is $1.26 \mathrm{~m}^{-1}$ in the far UV range at a wavelength $190 \mathrm{~nm}$ and falls to $.0100 \mathrm{~m}^{-1}$ at a wavelength of $320 \mathrm{~nm}$, near the transition to visible light [44]. 
In the visible spectrum, the absorption coefficient in water remains in the $.01 \mathrm{~m}^{-1}$ to $.02 \mathrm{~m}^{-}$

${ }^{1}$ range for wavelengths up to $550 \mathrm{~nm}$, corresponding to green light, beyond which it increases to $.2 \mathrm{~m}^{-1}$ at around $700 \mathrm{~nm}$ wavelength, corresponding to red light [45]. The low, nearly constant attenuation coefficient in the range between $320 \mathrm{~nm}$ and $550 \mathrm{~nm}$ is important because this contains the portion of the visible spectrum in which most Cerenkov photons are emitted. With the furthest detection distance considered of $8 \mathrm{~m}$ and the maximum attenuation coefficient of $0.02 \mathrm{~m}^{-1}$ for the photons of interest, the portion of the remaining intensity is $\mathrm{e}^{-.16}$, or 0.852 . This represents a maximum loss of $15 \%$ of the photons of interest through attenuation, so it has been deemed unnecessary to account for losses to attenuation beyond what is done automatically in the models.

\subsection{Dissertation Objectives}

The goal of this dissertation is to develop a power profile reconstruction method that will enable the application of novel forms of instrumentation in reactors with optically transparent coolants. A novel method is being designed that takes advantage of visual detectors external to the core to evaluate Cerenkov fluxes produced within the core. The system is modeled in MCNP to obtain estimates of the Cerenkov fluxes. The reconstruction method then takes the Cerenkov flux data and uses it to determine the spatial power profile of the reactor.

The method, if implemented, should be able to yield 2D power profile estimates of the core. It should also and have the ability to detect and help to identify various changes 
in reactor characteristics such as control rod movements, reflector movements, and coolant channel blockages.

The method can also use multiple sets of 2D data obtained by detecting light from each channel at different points above the core to obtain 3D power profile information in the form of axial flux tilt maps.

To accomplish the proposed objective, the following tasks will be performed:

- Develop a Cerenkov compatible 3D model of TRIGA reactor in MCNP

- Develop Cerenkov response function for electron fluxes in coolant channels

- Develop the power profile reconstruction method using electron flux measurements and response function data to estimate Cerenkov flux detection above core.

- Explore the ability of system to detect and distinguish different changes in reactor state, such as horizontal flux tilts caused by control rod movements or identifying a blocked coolant channels and locations.

- Develop 3D extrapolation capabilities by comparing viewpoints offset from the coolant channel to unfold the electron flux within a single channel to identify power tilts along the vertical axis or to determine the degree of blockage and axial location of a block in the channel.

\subsection{Impact}

The potential impact of this method is the addition of a new, non-invasive, lowfootprint form of instrumentation to characterize in-core conditions in nuclear reactors during operation. Many instrumentation methods measure a small number of locations in or near the core to obtain the total core power. Some next generation reactor designs could benefit from improved power profile determination. Reactor designs that use an optically 
transparent coolant with straight coolant channels are the most likely candidates for the use of visual instrumentation. A TRIGA reactor with a clear line of sight through the active core region or a FLiBe cooled reactor with straight coolant channels that run through the entire length of the core would be more easily measured using this system, while a pebble bed reactor would have no such line of visibility. A tightly packed PWR fuel assembly with grid spacers may not have straight segments through the assemblies, though water holes for control rods or spaces between assemblies may provide the necessary viewpoints. An opaque coolant such as liquid sodium would preclude the usage of the method developed here. Unlike gamma and neutron detectors, the light sensors can be located far outside of the active core region, and thus can avoid many of the issues with radiation that are present in most other forms of instrumentation. This could remove the cost of radiation hardening circuits or the need for instruments to be inserted and removed from the core. An additional measurement technique will also increase the diversity of instrumentation to further reduce the probability of all instruments failing. The visible light power measurements should be valid in the intermediate to full power range.

In some reactor designs, a visible instrumentation system could be installed outside of the core to provide large amounts of information about the power profile in the core without requiring any significant changes to the core. This information could be used to measure flux tilts from xenon oscillations, temperature irregularities, rods in shifted positions, blocked channels or other phenomena. In some cases, it would be possible to localize the disturbance to which region of the reactor and possibly even which assembly 
or coolant channel(s). This can provide operators with more information about what the cause of an issue may be.

A large amount of measureable information about the power profile in a reactor could also be used to validate reactor physics codes and models. This could be used to better optimize coolant flow distributions among assemblies, possibly contributing to power uprates. It could also be used to improve the algorithm for fuel loading and shuffling in the core during refueling operations, potentially increasing the discharge burnup slightly. These small improvements can still have a significant impact on reactor economics, particularly when compared to the simplicity and potentially low cost of the instrumentation system. 


\section{CERENKOV-BASED ANALYSIS METHODOLOGY}

\subsection{Production, transport and detection of Cerenkov radiation}

This method gathers information about the spatial power profile of a reactor by observing the amount of visible Cerenkov radiation coming out of the coolant channels or other coolant containing regions, such as inter-assembly spaces. Either a single multichannel photon detector or an array of photon detectors can be placed some distance above the core to observe the Cerenkov production in each channel.

The Cerenkov light above the core is caused by the Cerenkov production within a channel, which is directly proportional to the electron flux in the channel. The electron flux within a coolant channel is proportional to the gamma flux within the channel. In an operating TRIGA reactor, the gamma flux in a coolant channel is primarily caused by the fissions in the surrounding fuel pins. The fission rate density in fuel is proportional to the neutron flux. Thus, the Cerenkov observed above a channel is an indication of the power density and neutron flux within the surrounding pins. A diagram showing the progression from neutron flux to Cerenkov detection is shown in Figure 2. The only portion that involves a great distance is the transport of the Cerenkov photons through the coolant, which occurs in a straight line due to the wave nature of low energy light. 


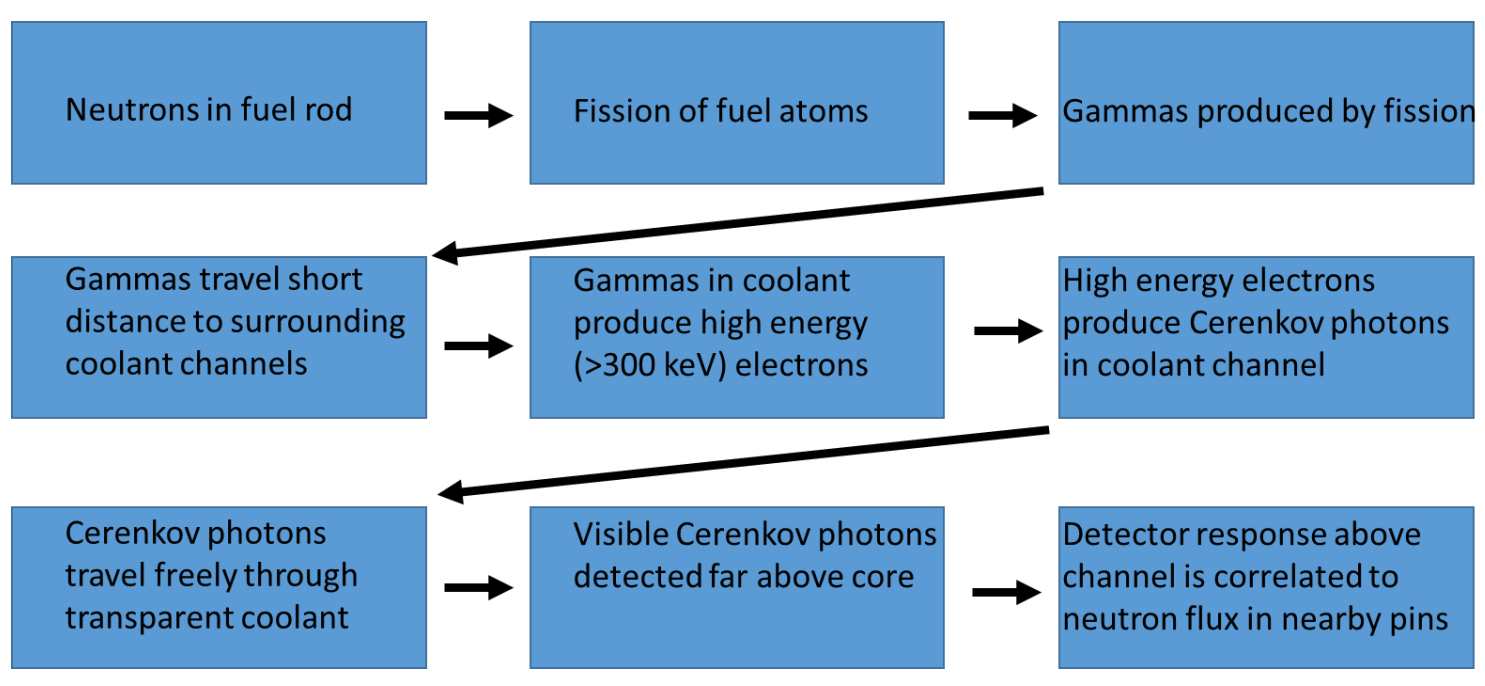

Figure 2 Progression from neutrons in the fuel to Cerenkov detection above core

The general steady state photon transport equation can be seen in equation 5 [46]:

$$
\mathbf{s} \cdot \nabla \Psi_{\text {photon }}(\boldsymbol{r}, \mathbf{s})=-\mu_{t} \Psi_{\text {photon }}(\boldsymbol{r}, \mathbf{s})+\mu_{s} \int_{4 \pi} \Psi_{\text {photon }}\left(\boldsymbol{r}, \mathbf{s}^{\prime}\right) P\left(\boldsymbol{s}^{\prime}, \boldsymbol{s}\right) d \Omega^{\prime}+S(\boldsymbol{r}, \boldsymbol{s}),
$$

where $\mathbf{r}$ is the position vector in space, $\mathbf{s}$ is the unit direction vector, $\Psi_{\text {photon }}(\mathbf{r}, \mathbf{s})$ is the photon flux at point $\mathbf{r}$ in direction $\mathbf{s}, \mu_{\mathrm{t}}$ is the attenuation coefficient (similar to the total macroscopic cross section $), \mu_{\mathrm{s}}$ is the scattering coefficient, $\mathrm{P}\left(\mathbf{s}^{\prime}, \mathbf{s}\right)$ is the probability of a photon with direction $\mathbf{s}$ ' scattering into solid angle $\mathrm{d} \Omega$ around direction $\mathbf{s}$, and $\mathrm{S}(\mathbf{r}, \mathbf{s})$ is the spatially and angle dependent photon source term. The medium in which the photons are propagating and being produced is assumed homogenous.

For Cerenkov photons, the photon source term $\mathrm{S}(\mathbf{r}, \mathbf{s})$ can be approximated by equation 6:

$$
\mathrm{S}(\mathbf{r}, \mathbf{s})=\frac{1}{4 \pi} \int d E^{\prime} \Phi_{\text {Electron }}\left(\boldsymbol{r}, E^{\prime}\right) \Sigma_{\text {Cerenkov }}\left(E^{\prime}\right)
$$

where $\Phi_{\text {Electron }}(\mathbf{r}, \mathrm{E})$ is the spatial and energy dependent scalar electron flux. The quantity $\Sigma_{\text {Cerenkov }}(\mathrm{E})$ is the energy dependent macroscopic cross section for Cerenkov production 
by electrons, equal to the number of Cerenkov photons produced by an electron of energy E per unit path length. This approximation adds the assumption that the electron flux is isentropic.

To find the Cerenkov flux at a specific point $\mathbf{r}$ with photons within an angle range $\mathrm{d} \Omega$ of a specified direction $\mathbf{s}$, a solution to some form of equation 5 must be obtained. This flux only depends on photons produced or scattered in the region contained by the a cone of angle $d \Omega$ opening away from the point $\mathbf{r}$ in the direction $-\mathbf{s}$. Viewing a coolant channel from a significant distance above the core provides a very small angle view of a thin, constant width region that produces Cerenkov photons. In such a situation where the angle range $d \Omega$ is small and the cross sectional area of the region contributing to the flux is both small and constant, this can be approximated by a line integral starting at $\mathbf{r}$ in the direction of $-\mathbf{s}$. This is a simplification and solution of equation 5 and can be seen in equation 7 :

$$
\Psi_{\text {photon }}(\boldsymbol{r}, \mathbf{s})=\int_{0}^{\infty}\left[\int_{4 \pi} \Psi_{\text {photon }}\left(\boldsymbol{r}-r \boldsymbol{s}, \mathbf{s}^{\prime}\right) P\left(\boldsymbol{s}^{\prime}, \boldsymbol{s}\right) d \Omega^{\prime}+S(\boldsymbol{r}-r \boldsymbol{s}, \boldsymbol{s})\right] \frac{e^{-\mu_{t} * r}}{r^{2}} d r .
$$

The variable $r$ (not bold) represents the distance from the point $\mathbf{r}$. The $\exp \left(-\mu_{\mathrm{t}}{ }^{*} \mathrm{r}\right)$ term accounts for attenuation, while the $1 / \mathrm{r}^{2}$ term is for the dissipation over distance from a point source.

The Cerenkov photons being detected have wavelengths in or very near the visible range, where light behaves less like a particle. The scattering of visible photons is relatively small, so an approximation is made such that the scattering of visible photons into the angle range is neglected as a source of photons contributing to the flux, and is only accounted for as part of the attenuation term, $\mu_{\mathrm{t}}$. This allows the scattering term in equation 7 to be dropped, leaving only the source term, the attenuation and the $1 / \mathrm{r}^{2}$ 
dependence. Inserting equation 6 into equation 7, applying the aforementioned approximations yields equation 8:

$$
\Psi_{\text {photon }}(\boldsymbol{r}, \mathbf{s})=\int_{0}^{\infty} d r \frac{1}{4 \pi} \int d E^{\prime} \Phi_{\text {Electron }}\left(\boldsymbol{r}-r \boldsymbol{s}, E^{\prime}\right) \Sigma_{\text {Cerenkov }}\left(E^{\prime}\right) \frac{e^{-\mu_{t} * r}}{r^{2}}
$$

This can be approximated by a discretization that slices the region where significant contributions to the Cerenkov flux are made into segments and separating the energy dependent electron flux into bins. Doing this yields equation 9:

$$
\Psi_{\text {photon }}(\boldsymbol{r}, \mathbf{s})=\frac{1}{4 \pi} \sum_{n=1}^{E \text { bins }} \sum_{m=1}^{\text {Segments }} \Phi_{\text {Electron }}\left(z_{m}, E_{n}\right) \Sigma_{\text {Cerenkov }}\left(E_{n}\right) \frac{e^{-\mu_{t} * r_{m}}}{r_{m}^{2}} .
$$

Another simplification is made that combines the $1 / 4 \pi$, the Cerenkov production term, the exponential attenuation and the $1 / \mathrm{r}^{2}$ term into a single Cerenkov response function. This response function is a correlation between the electron flux in a bin and the amount of Cerenkov light that reaches the detection point. The response function is very similar to a cross section, because it is the amount of Cerenkov detected at the detection point per unit of electron path length in the specified region of the channel. Adding this simplification transforms equation 9 into equation 10:

$$
\Psi_{\text {photon }}(\boldsymbol{r}, \mathbf{s})=\sum_{n=1}^{E \text { bins }} \sum_{m=1}^{\text {Segments }} \Phi_{\text {Electron }}\left(z_{m}, E_{n}\right) \Sigma_{\text {Response }}\left(z_{m}, E_{n}\right)
$$

where $\Sigma_{\text {Response }}$ is the aforementioned response function.

The determination and verification of the response cross sections is covered in the Electron to Cerenkov flux correlation section. Equation 10 and the correlation factors are used to determine the amount of Cerenkov detected above the core without requiring the computationally expensive and potentially problematic simulation of Cerenkov photons in the whole core. 


\subsection{Modelling Cerenkov in TRIGA}

MCNP 6.1.1 Beta is the primary tool used to model the reactor. This version of MCNP has the added capability to model photons with energies in the visible range, the use of indices of refraction, and the ability to produce and track Cerenkov radiation [47]. The Cerenkov production is governed by a discretized form of the Frank Tamm formula shown in equation 11 :

$$
N=2 \pi \alpha L z^{2} \sum_{i=1}^{I}\left(\frac{1}{\lambda_{i}}-\frac{1}{\lambda_{i-1}}\right)\left(1-\frac{1}{\beta_{i}^{2} n_{i}^{2}}\right)
$$

Here $\mathrm{N}$ is the number of photons emitted, $\alpha$ is the fine structure constant, $\mathrm{L}$ is the total path length, $\lambda$ is the wavelength, $\beta$ is the ratio of the charged particle's speed to the speed of light and $\mathrm{n}$ is the frequency dependent index of refraction. Los Alamos has done verification of the Cerenkov production in MCNP6.11 Beta to ensure that it does correctly produce the number of photons predicted [48].

In this research effort, methods that directly produce, track and tally Cerenkov photons in a model of the reactor are used, as well as methods that only track neutrons, gammas and electrons and rely on correlations to estimate the resulting Cerenkov fluxes. A model of the TRIGA has been developed to use in the Cerenkov analysis. An axial cross section of the core from the model can be seen in Figure 3. The aqua colored background is water. The green blocks in the left and right portions of the picture are graphite reflector blocks. The blue circles are the standard fuel pins. The green circle near the right side of the $2^{\text {nd }}$ row is the regulating rod, the 4 other green circles are the safety shim control rods. The orange circle in the middle of the core is the transient control rod. The four large 
orange circles at the bottom of the picture are hollow chambers used for sample irradiations and other experiments.

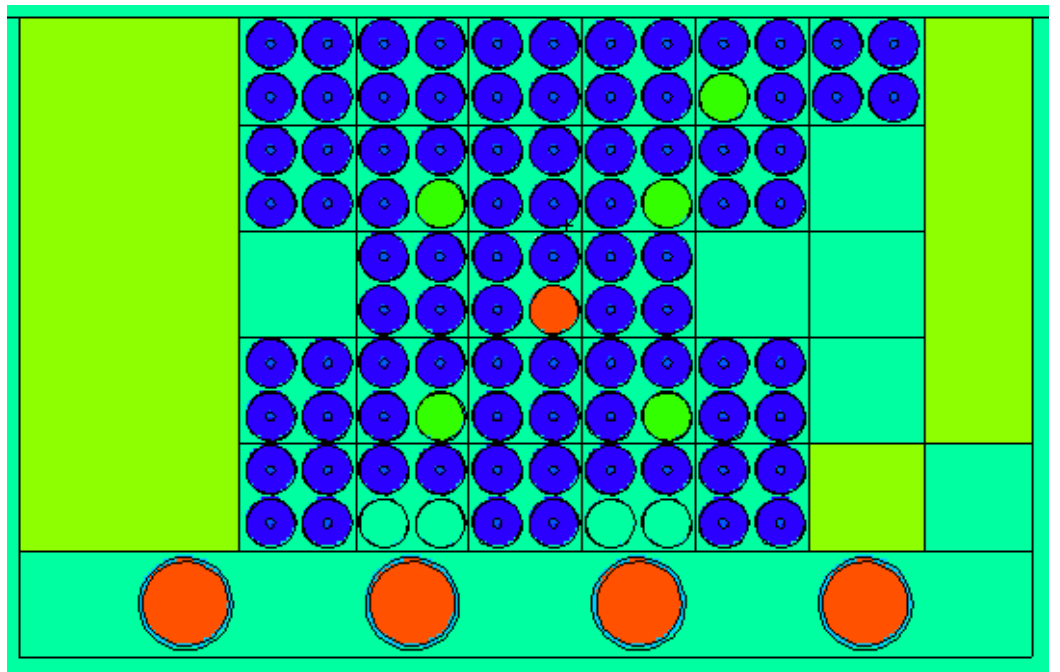

Figure 3 Axial (XY) cross section of the TRIGA core.

This model was created without using any universe or lattice structures, due to an issue with refraction in MCNP 6.11 beta that causes lost particles when multiple universes are present. As is, each pin and structure is defined individually, with its own unique surfaces and cells. The reactor is oriented with the $\mathrm{z}$ axis corresponding to the vertical axis of the core and the $+y$ direction corresponding to the side of the reactor facing the graphite coupler box. The cells and surfaces for the pins and control rods are numbered based upon which position in the lattice they occupy. The general system for surface and cell designation used is AYYXXBB, where $\mathrm{A}$ and $\mathrm{BB}$ are used to denote what the cell or surface is, such as fuel meat, cladding, a top cap, etc. In many of the pin specific cells, digit $\mathrm{A}$ is not included; it is primarily used for denoting different water regions, and $\mathrm{BB}$ may only contain one digit for the first 9 cells. The digits for BB generally indicate which 
radial region of a fuel pin the surface or cell involves, such that 1 is cylinder bounding the inner zirconium rod, 2 is the fuel bound, 3 is cladding, etc. The digits for XX denote the $\mathrm{X}$ position of the cell in the lattice, while YY denotes the y position. For example, the $4^{\text {th }}$ pin in the $3^{\text {rd }}$ row is denoted as pin 3,4 . The fuel region in this pin is cell 03042 , where the 2 denotes the $2^{\text {nd }}$ radial region of the pin. 03041 is the central zirconium rod.

The reactor model contains 86 regular fuel pins filling some of the locations in a $12 \times 10$ lattice. Each fuel pin was modeled with a zirconium rod in the center, surrounded by the $\mathrm{Zr}-\mathrm{H}$ fuel, with stainless steel cladding. Graphite regions are placed axially above and below each fuel pin within the cladding and aluminum caps are at the top and bottom of each pin. There are four safety shim control rods in the $(4,4),(4,8),(8,4)$, and $(8,8)$ locations in the core. The safety shims are moveable rods that consist of a fuel region similar to the fuel pins with a boron carbide absorber region above it. The safety shims are fuel following to increase their reactivity worth, and are used to make large reactivity adjustments in the core. A vertical cross section of the $4^{\text {th }}$ row of pins, showing fuel rods and two of the safety shims can be seen in the left side of Figure 4. The transient control rod is located in the center of the core at location $(6,6)$. This is modeled as a moveable cylinder of boron carbide with aluminum endcaps within stainless steel cladding with air regions above and below it. The length of the boron region is the same as that of the fuel pins. When the transient rod is moved, the boron region with its caps moves within the air region, while the rest of the rod remains in place. The transient rod extends above the fueled region of the core such that when the transient rod is withdrawn the bottom of the absorber is slightly above the top of the fueled regions of the fuel rods. A vertical cross 
section of row 6 , displaying the transient rod at full withdrawal can be seen in the middle section of Figure 4. The regulating rod is a moveable control rod at location $(2,9)$ in the second row of fuel pins. This control rod is not fuel following and is located near the periphery of the core so that it has a lower total reactivity worth. The regulating rod is primarily used for small reactivity changes and is usually adjusted automatically to regulate the reactor power and maintain it at a set level. A translation card in the end of the input deck can be used to adjust the degree to which the control rods are inserted or removed. The position designation of control rods ranges from $0 \mathrm{~cm}$ when fully inserted to $40 \mathrm{~cm}$ when fully withdrawn.
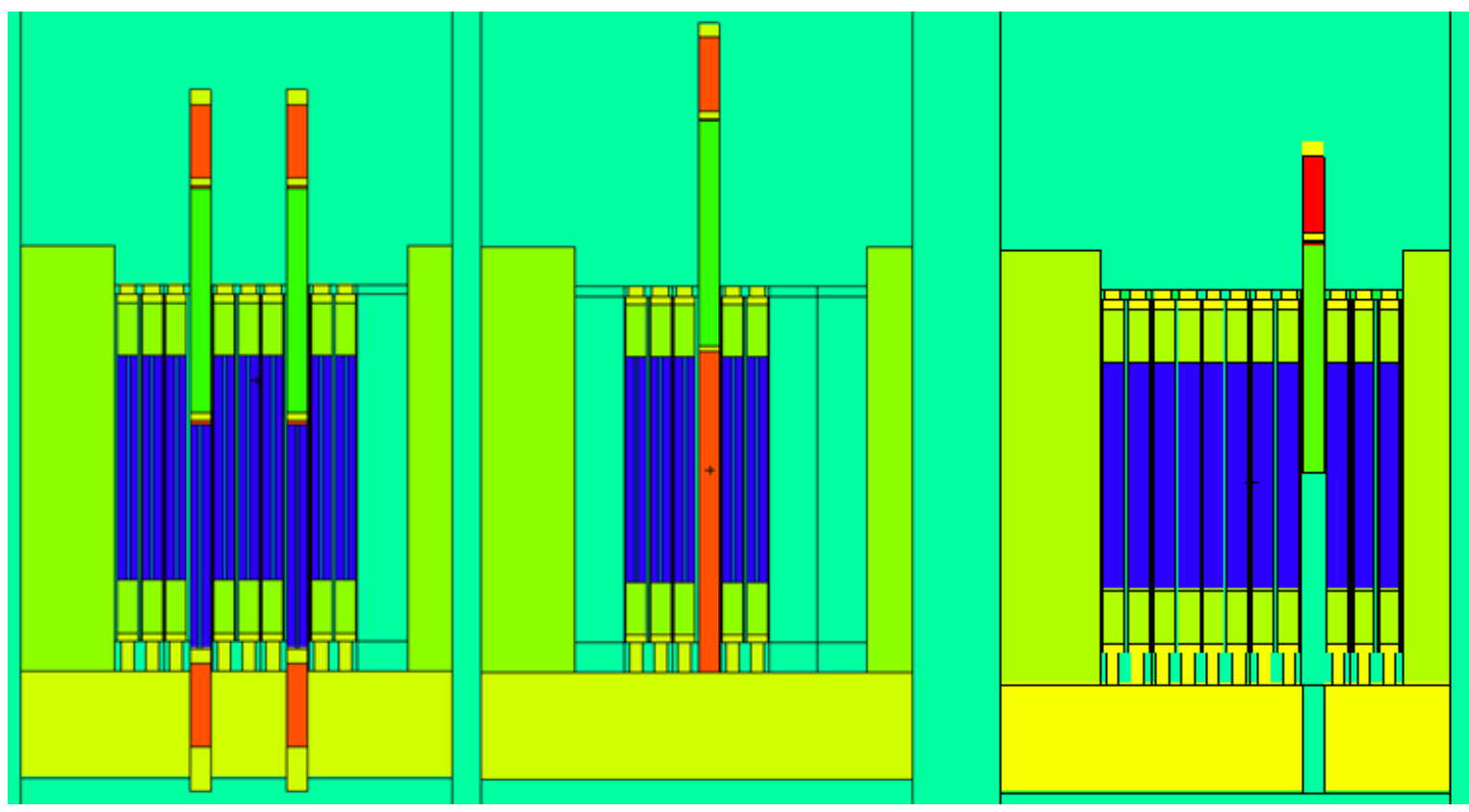

Figure 4 Vertical cross sections of row 4 (left) and row 6 (middle) and row 2 (right) displaying fuel pins, safety shims, the transient control rod, and the regulating rod 
The core has graphite blocks beside it in the $+\mathrm{x}$ and $-\mathrm{x}$ directions that act as reflectors. In this model, they are approximated as continuous rectangular prisms without anything but graphite inside of them. The lower grid plate and the structures by which the pins are supported by the plate were approximated as a solid aluminum block with a few simple square and cylindrical holes in it for control rods and other tubes and instruments. The four irradiation tubes next to the core in the $-\mathrm{y}$ direction are also modeled as cylinders of air with steel cladding. Due to the way that MCNP calculates geometries and transports particles, cells with large numbers of bounding surfaces can drastically increase runtime, so it was necessary to place planes to divide the water regions in the core into smaller regions that each contained only 4 pins to reduce the runtime.

One of the primary sources of error in the criticality estimation in this model is the usage of a single homogenously burned and heated material for all of the fuel pins. Differences in the modeling of the materials and regions inside of the graphite block on the $-\mathrm{x}$ side of the core also contribute to a difference in the k-eff estimates produced by this model. The higher fidelity lattice-based neutron-only input deck from the NSC with many separately depleted zones in each fuel pin and a more detailed treatment of the graphite source region is used when more precise criticality estimates are needed. The more complex deck cannot be used for Cerenkov production due to the lattice lost particle issue. Using the neutronics only model, the reactor is critical with the transient rod fully withdrawn, the safety shims withdrawn $28.4 \mathrm{~cm}$ and the regulating rod withdrawn by 19.8 $\mathrm{cm}$. Modeling the reactor with these rod positions in the Cerenkov compatible model produces a k-eff estimate of $1.00556 \pm .00037$. This degree of deviation, while significant 
from an operational standpoint, is acceptable for the modeling of Cerenkov fluxes, as MCNP will run the number of neutrons specified when using a kcode regardless of the actual criticality value. Despite a difference of nearly $1 \$$ of reactivity, the difference in photons produced is proportional to the difference in the number of fissions, and should therefore be on the order of $0.5 \%$. However, due to this discrepancy, the more accurate lattice based model with heterogeneously burned fuel is still used for determining different critical rod positions.

\subsection{Electron flux to Cerenkov correlations}

An analysis technique has been developed that uses spatial and energy dependent electron flux measurements to estimate the Cerenkov fluxes at a point directly above the core using a correlation matrix. The correlation factors are a discretization of the response function, $\Sigma_{\text {Response }}\left(\mathrm{Z}_{\mathrm{m}}, \mathrm{E}_{\mathrm{n}}\right)$, from equation 10 . The correlation relates the electron flux within a space with an energy within a specific range to the resulting visible Cerenkov flux at a distant point. A correlation is generated for 23 electron energy bins ranging from $200 \mathrm{keV}$ to $10 \mathrm{MeV}$ and 16 spatial bins. The spatial region considered for the tally is a $1 \mathrm{~cm}$ radius cylinder in the coolant channel, nearly touching the 4 neighboring pins. This region in relation to the surrounding pins can be seen in Figure 5. 

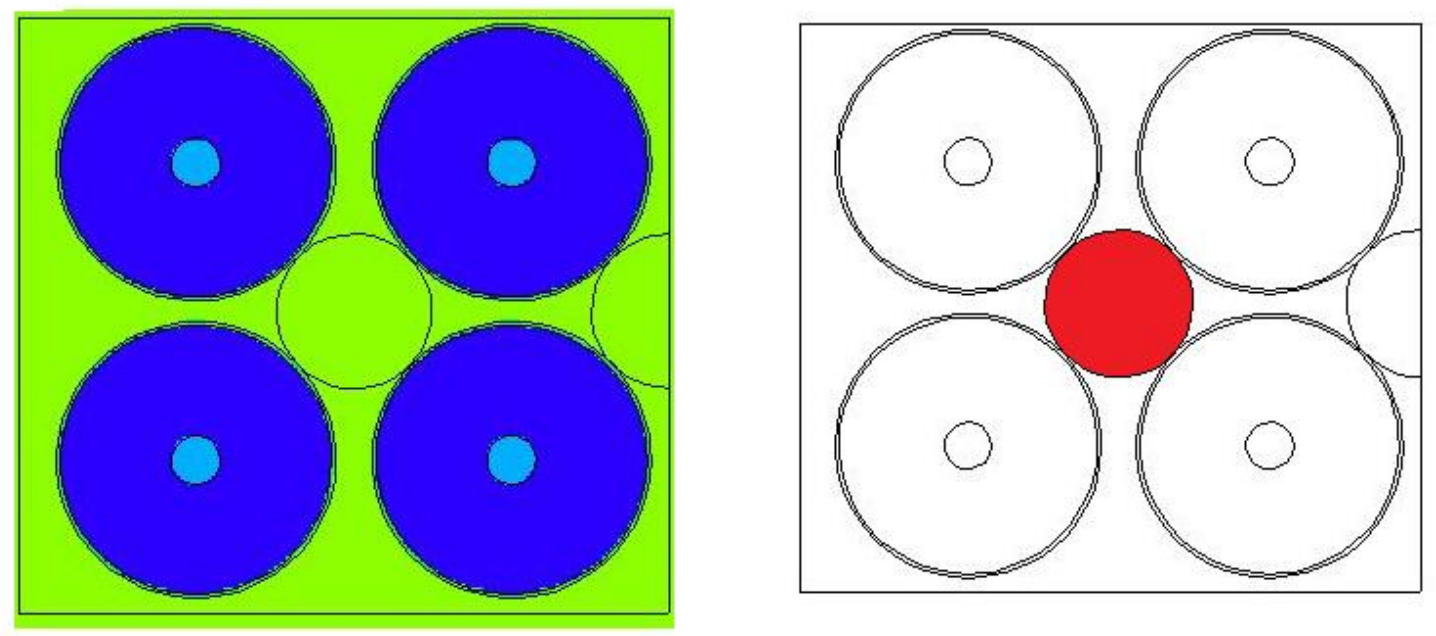

Figure 5 XY cross sections of 4 pin model showing pins, coolant and the reflecting boundaries. (Right) the red region is the portion of the channel containing the electron tallies.

The cylindrical region between the pins is segmented into 16 spatial bins along the vertical (z) axis, each $4 \mathrm{~cm}$ long, ranging from $\mathrm{z}=-32 \mathrm{~cm}$ to $\mathrm{z}=32 \mathrm{~cm}$ below and above the core midplane. This produces a total of $16^{*} 23=368$ bins for which a correlation is found. The Cerenkov flux is tallied at a location $200 \mathrm{~cm}$ above the core midplane and only counts photons with energies between $1.76 \mathrm{eV}$ and $3.17 \mathrm{eV}$, corresponding to visible light. In Figure 6, a diagonal cross section of the 4 pin model can be seen, showing two of the pins and the coolant channel. As an example, one segment is singled out in the right side of the figure. 

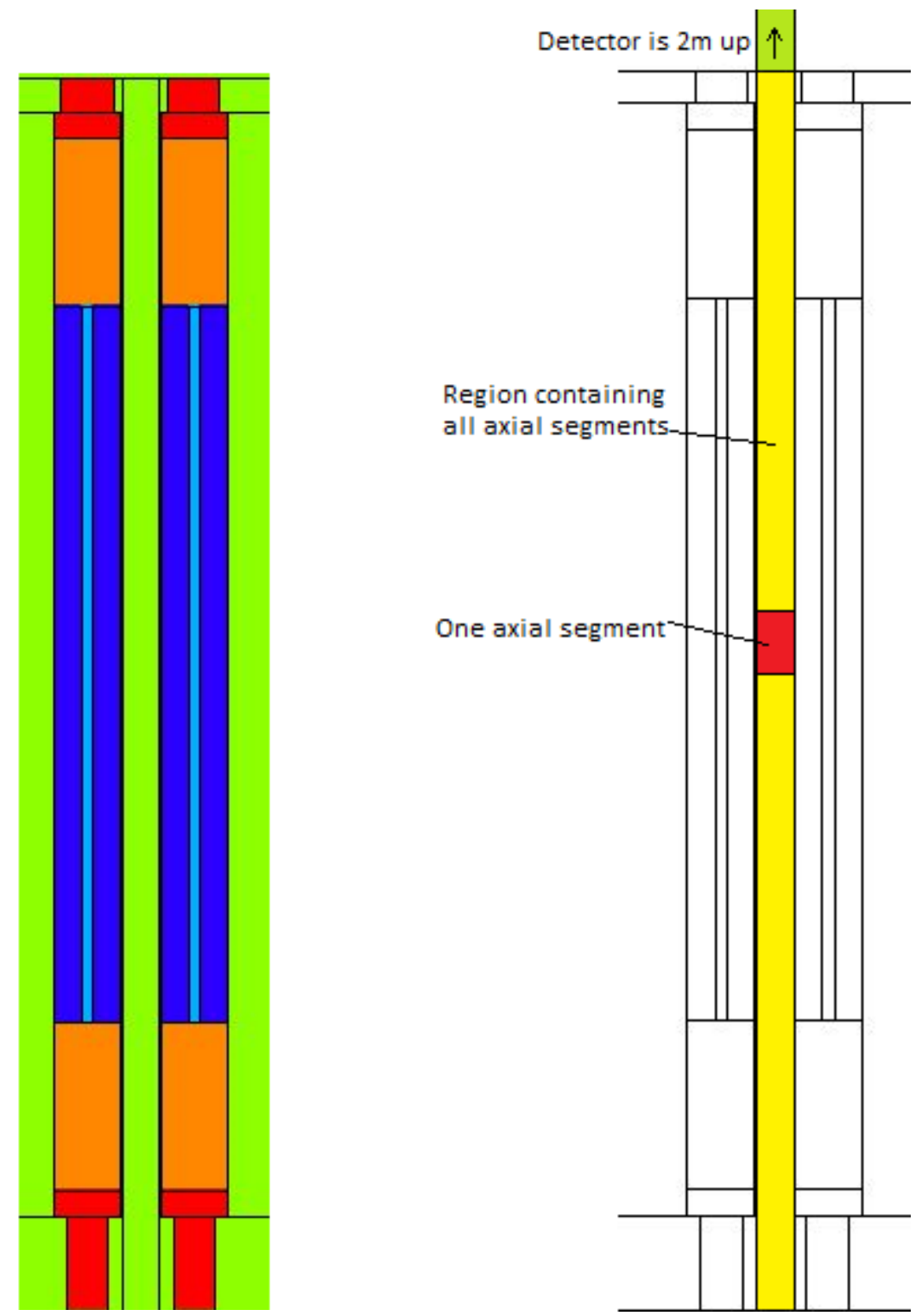

Figure 6 Diagonal X/Y vs $Z$ cross section showing 2 of the pins and the cylindrical region of the channel being tallied. (Left) color corresponds to materials so that the coolant and fuel pins can be seen. (Right) the red region corresponds to one axial segment being tallied, while the yellow region contains the other 15 axial segments tallied in separate MCNP runs. The green region shows the bottom of the column of water above the channel where the Cerenkov detection plane is located, $2 \mathrm{~m}$ above the core's axial centerline. 
A separate, simple model is run with only photons and electrons to obtain reasonably precise correlation factors. This model consists of a tube of water representing a coolant channel and the region above it surrounded by regions of zero photon importance in which photons are killed. The zero importance region must be made of water in the model instead of vacuum to prevent total internal reflection effects from the differing indices of refraction. The white regions of the right side of Figure 6 are zero photon importance in this model. The electron importance is set to zero everywhere except for in the spatial region that the correlation is being calculated for, and the electron physics card is set to kill all electrons with energies below the lower energy bound of the bin for which the correlation factor is being determined. In Figure 6, the red region would be the only region with a nonzero electron importance for a run producing correlations for that segment. Then, the source definition card is set to homogenously spawn electrons in the region of interest with an initial energy distribution that approximately results in a flat energy profile within the energy bin range. The electron flux is tallied to ensure that only electrons within the energy bin are present and that there is a close to flat energy profile within the bin. A photon tally counts the photons crossing the plane at $\mathrm{z}=200 \mathrm{~cm}$. The tally used only counts photons with energies in the visible range travelling in a direction that is within a $\mathrm{mu}=.99995$ cone of the $+\mathrm{z}$ direction. The pseudo-arbitrary angle restriction exists to screen out photons that have come from regions of the core other than the coolant channel of interest and to mimic the way a real photon detector such as a camera would be able to distinguish where the light is coming from using lenses and small apertures. The results of this tally provide an estimate of the visible Cerenkov flux, which 
is then divided by the result of electron flux tally to get the correlation for the Cerenkov flux produced per unit of electron flux in the spatial and energy bin used in the model. This is done for all 368 combinations of energy ranges and spatial segments in the coolant channel to get what acts as a spatial and energy dependent response function for electrons in the coolant producing an observable Cerenkov response $2 \mathrm{~m}$ above the core. A table containing the ratio of the photon flux above the core to the electron flux within a segment and the corresponding Monte Carlo uncertainty for each of the 368 spatial and energy bins can be seen in Appendix A. The python scripts used for this process can be found in Appendix C.

The set of correlations between the electron and photon fluxes can then be used to estimate the Cerenkov flux without requiring the actual production and tracking of Cerenkov photons. To verify this, a model consisting of only 4 pins in an infinite lattice is used. The first version of the model is run using a kcode criticality source with neutrons, electrons and photons tracked, but no Cerenkov production. This model uses a surface source write (SSW) card that records all of the gammas and electrons passing out of the cladding and into the coolant. Then a second model is run with the same geometry with Cerenkov production enabled and zero photon importance in all materials except the coolant to simulate opacity, because MCNP611 has some issues where visible photons will travel almost unimpeded through opaque materials such as steel or fuel. This second model uses a surface source read (SSR) card to take the gammas and electrons leaving the pins and transport them through the coolant to produce electrons and Cerenkov photons. The second model tallies the electrons in the $1 \mathrm{~cm}$ cylinder comprising most of the coolant 
channel with spatial and energy bins corresponding to the correlations previously calculated. The electron tally results for each spatial and energy bin are then multiplied by the correlation factor for that bin to obtain its contribution to the Cerenkov flux above the reactor. The contributions from all of the bins are added together to estimate the total Cerenkov observed above the core. The resulting estimate of the Cerenkov flux at $200 \mathrm{~cm}$ above the core midplane is $5.46 \mathrm{E}-6$, normalized to the number of source neutrons from the SSW model. To verify the consistency of this method, the second model also tallies the Cerenkov photons crossing a plane at $\mathrm{z}=200 \mathrm{~cm}$. The Cerenkov flux from the direct tally is $5.919 \mathrm{E}-6$ per source particle. This means that $92 \%$ of the observed photons tallied above the core were accounted for in the electron tally in the coolant channel. The less than $100 \%$ result is expected, because the regions outside of the cylindrical electron tally also contribute some to the flux directly above the channel. The $92 \%$ estimate result is considered sufficient verification that the Cerenkov response function based method developed here is internally consistent with MCNP's Cerenkov tracking model for finding the Cerenkov flux at the plane of interest above the core.

The degree of correlation between the Cerenkov produced in a channel and the power output of the 4 neighboring pins is also investigated using the 4 pin verification model. To do this, the SSW deck was run to record all of the gammas and electrons leaving the pins, and then two different decks are run using SSR. The first SSR deck has vacuum/zero importance boundary conditions in the planes separating the 4 pins from where their neighbors would be. This allows the electron flux and thus Cerenkov production in the coolant channel to be measured with only the 4 surrounding pins 
contributing to it. This is then compared to a second case in which reflecting boundaries are used to create an infinite 2D lattice of pins. Then, the electron flux in the channel is the result of the 4 pins around the channel, and an infinite number of pins beyond those 4 , which is an estimate of a maximum bound of what can be contributed by the rest of the reactor. Figure 7 shows representations of the two cases.
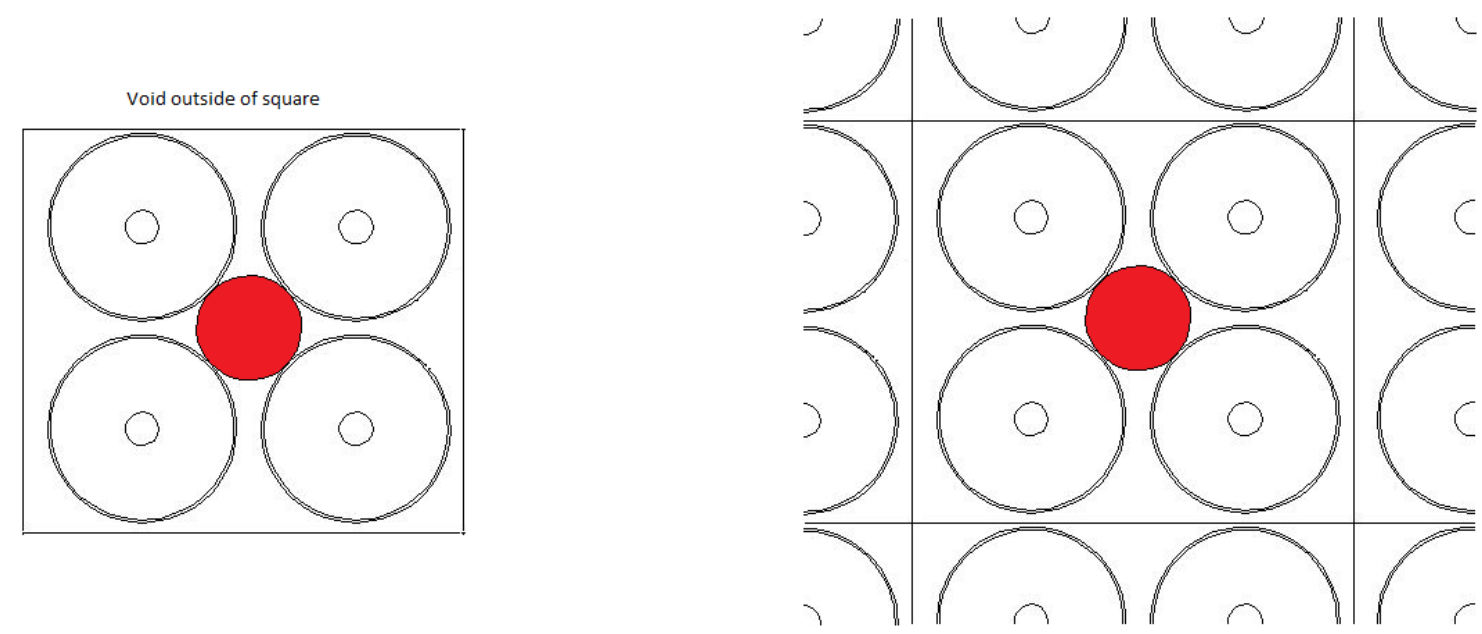

Figure $7 \mathrm{XY}$ cross sections of two versions of the $\mathbf{4}$ pin model, with the electron tally in the red region. (Left) has only 4 pins with a void around it, such that only gammas from those 4 pins can enter the channel. (Right) is reflected, such that the gammas reaching the coolant channel to produce electrons can come from an infinite lattice of pins.

The results for the source particle normalized total electron flux in the channel for the 4 pin and infinite lattice cases were $8.935 \mathrm{E}-5$ and $9.794 \mathrm{E}-5$ with $0.5 \%$ uncertainties. This non-reflected 4 pin model's electron flux is smaller than the infinite lattice's by a factor of 0.913 , meaning approximately $91.3 \%$ of the electrons in the channel are caused by fissions in the 4 surrounding pins, and only $8.7 \%$ are from the rest of the reactor. This is deemed an acceptably strong correlation between the Cerenkov flux observed above a channel and the power density in the surrounding pins. This calculation does use an 
approximation of a flat power density in an infinite lattice of pins. Therefore it is possible that in the real, finite reactor that the contribution form the pins bordering a channel could be less than $90 \%$ of the total in cases where the power density in those pins is significantly less than in their neighbors, such as near the edge of the reactor. There will also be some difference in the channels that are only bordered by 2 or 3 pins. However, the strength of the correlation in the infinite to 4 pin comparison was deemed sufficient such that the large number of other outlying cases need not be analyzed.

\subsection{Two dimensional Cerenkov mapping}

The spatial power profile information is primarily obtained by making a $2 \mathrm{D}$ map of the Cerenkov radiation coming from the coolant channels in the reactor. Due to the irregular and asymmetric nature of the reactor, some periphery coolant channels are not tallied, but instead a 9x9 grid of coolant channels is used for the investigation. All of the fuel pins in the reactor, as well as one of the graphite reflector blocks can be seen in Figure 8 along with additional notations for the location of control rods and the electron tallies used for the Cerenkov response estimations. The safety shims are marked "Shim", the transient control rod marked "TR" and the location of the regulating rod marked "RR". The small circles between the pins are the portions of the coolant channels that are tallied. The red box shows the extent of the area covered by the Cerenkov map. The 4 pins in the top right corner of the reactor are excluded from the map to keep a rectangular region while avoiding having to deal with the presence of the graphite block at the bottom right corner. The coolant channels that border the left edge of the reactor are similarly excluded 
to avoid collision with the graphite reflector. Those bordering the top or $+y$ direction face of the core are excluded to avoid issues with moving the core nearer to the graphite coupler and potential collision problems when the coupler is close to touching the core. The bottom face is left out to maintain a more symmetrical arrangement with a square 9x9 grid.

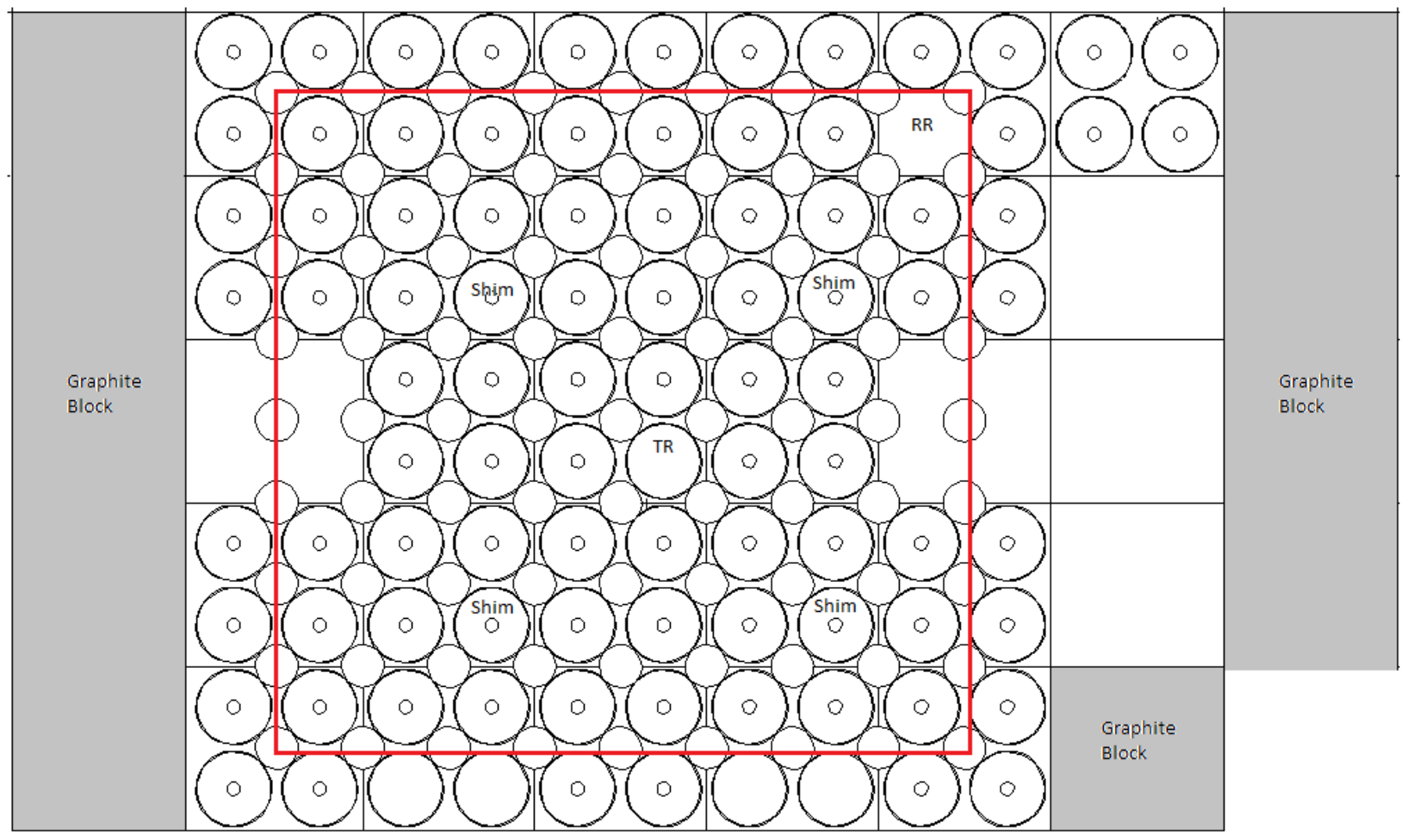

\section{Figure 8 XY cross section of core showing coolant channels tallied for Cerenkov map and control rod positions. The red box corresponds to the region that is mapped in the subsequent power profiles.}

The correlations relating the electron flux in a specific axial segment of a channel with a specific energy range to the amount of Cerenkov produced $2 \mathrm{~m}$ above the core are used to calculate the total Cerenkov response for each coolant channel. In MCNP, the electron flux in each channel is tallied and binned by energy and axial position, and then each bin is multiplied by its correlation factor to determine its contribution to the total 
Cerenkov. Then all of the bins' Cerenkov contributions for a given channel are added together to obtain an estimate of the total Cerenkov flux that would be observed above that channel within the specified angle range. Though the relative uncertainties for individual electron flux bins are high, the uncertainty for the total Cerenkov in a channel is usually very low, due to uncertainty propagation. This is done for each of the 81 tallied coolant channels and the results for each channel plotted as seen in Figure 9.

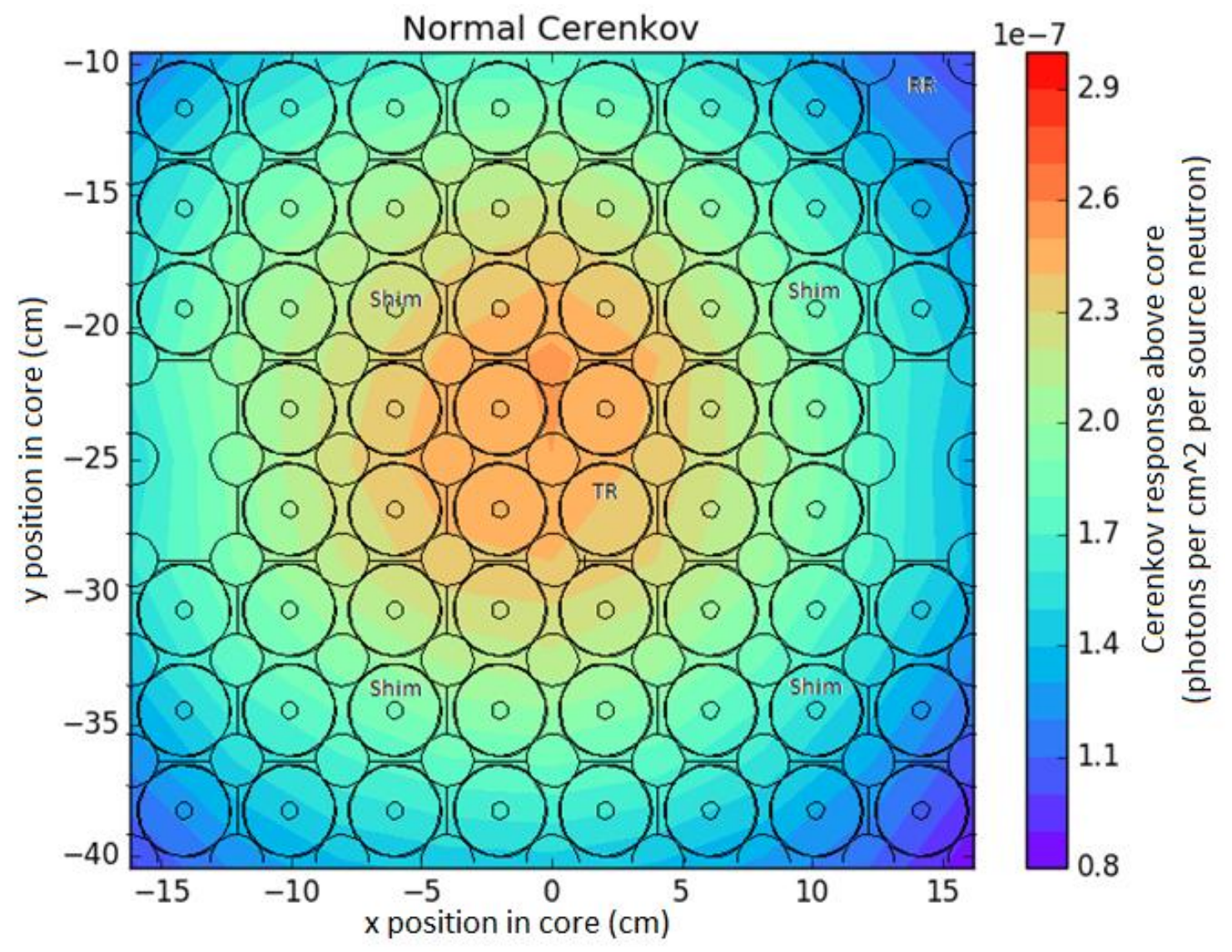

Figure 9. This plot shows the Cerenkov estimates $2 \mathrm{~m}$ above the core, with and outline of the XY geometry of the core overlaying the image. The safety shims, transient rod (TR) and regulating rod (RR) positions are labeled. In this figure, the reactor is in the normal baseline critical state, with the regulating rod at $19.8 \mathrm{~cm}$, the shims at $28.4 \mathrm{~cm}$ and the transient rod fully withdrawn. This is used as a baseline to compare with other perturbed reactor states. The scale of the plot is the normalized Cerenkov flux per fission neutron. 
This is a smeared estimate of the core's $2 \mathrm{D}$ power profile, as approximately $90 \%$ or more of the Cerenkov from each channel is caused by the 4 pins surrounding the channel. The advantage of this power profile estimate is that it can be obtained relatively easily, and does not require placing 81 detectors within the core, but could involve a single camera type detector using redirection through mirrors, lenses, or fiber optic cables, or using correction factors for the shadowing effects of channels not directly below the detector. The mirrors, lenses or fiber optic cables could re-direct the light from above each channel to a single multichannel photon detector, with the light from each coolant channel being detected by a different channel on the detector. The use of correction factors for shadowing could allow estimates of the total Cerenkov directly above a channel to be made by multiplying the flux at the detector by a factor based upon what portion of the channel is visible to account for the shadowing effect. It could require more than one detector depending on how far away from the core the detection plane is and the size of the core, but still far less than one detector per channel. The limiting factor is whether or not the detector has sufficient line of sight to the channel to give an accurate estimate of the Cerenkov produced in the channel without introducing unacceptable errors. If a view that at least reaches to the bottom of the channel is required, then at $2 \mathrm{~m}$ above the TRIGA reactor, nine detectors should be sufficient to get an accurate determination of the Cerenkov map without requiring any additional light redirection. A single detector or camera could be used if placed at least $6.5 \mathrm{~m}$ above the midplane, which is still below the surface of the pool located approximately $8 \mathrm{~m}$ above the midplane. 
In Figure 10, a graphic depicting the placement of either an array of detectors, or a single detector is shown. A simple experiment could be performed with a setup similar to the right side, using a single camera positioned far above the core, such that the angle between each channel and the viewpoint is still close enough to vertical for a sufficient portion of the channel to be visible. The models use a setup similar to the left side, with many detectors, using an assumption that the Cerenkov flux is observed directly above each channel. While this may be impractical in a real situation, the idealization makes the calculation of the Cerenkov fluxes simpler by not requiring various different corrections for shadowing effects, and allows the data to be more directly used to produce a flux map. For attempts at experimental verification, a single camera positioned far above the core, similar to the right side of Figure 10, would be more likely, as this would be easier and less expensive to set up. 


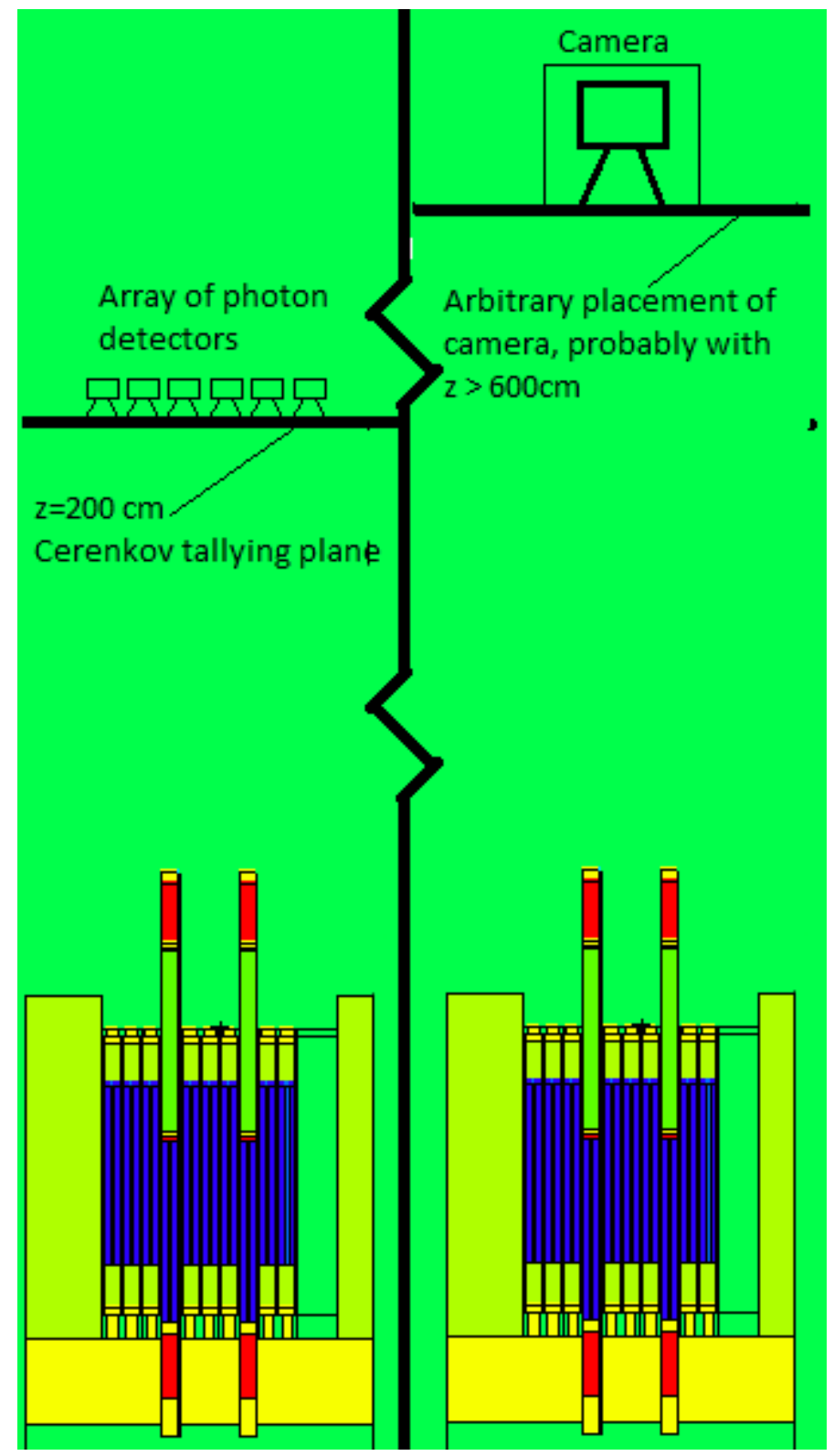

Figure 10 Two possible ways to measure the Cerenkov flux above the core. An array of photon detectors (left) or a single, multichannel detector or camera (right).

\subsection{Reactor state change analysis with $2 D$ Cerenkov maps}

One of the main metrics of the Cerenkov detection system is its ability to detect and identify perturbations in the reactor relative to a baseline, "normal" state. Various 
alterations to the state of the reactor are analyzed, all maintaining criticality with the same fuel temperature and power level. As the total power in the core remains constant, the observable changes will be in the power tilts and the power in specific regions of the reactor relative to others.

Due to the uncertainties in Monte Carlo simulations, relatively large changes in the reactor are investigated, to ensure that the differences are noticeable amid the noise. $\mathrm{K}$-eff in the model used is approximately 1.006 for the normal critical state. This is slightly higher than 1 because the homogenization of the fuel burnup provides an increase in reactivity, but in the more complex, neutronics only model, the rod positions used do bring k-eff to 1 . In this baseline case, the shims are withdrawn $28.4 \mathrm{~cm}$ and the regulating rod withdrawn $19.8 \mathrm{~cm}$. This is the "normal" reactor state that is used as a comparison for the analysis of the perturbed states. In the altered reactor states, the safety shims are readjusted to maintain the same level of criticality in the reactor, with the model's k-eff estimate again being close to 1.006 . An alteration that inserts negative reactivity will thus have the safety shims slightly withdrawn to provide enough positive reactivity to counteract the change. This leads to an effect where a change in the power at one location causes a small opposite change throughout the rest of the core. To maintain consistency between different comparisons, the same scale is used for all Cerenkov map plots, absolute difference plots and relative difference plots. This causes some effects to be harder to distinguish in some plots, but the usage of both absolute and relative flux comparisons helps to circumvent this issue. 
The first state change analyzed is the partial removal of the regulating rod, located near the edge of the reactor. The regulating rod is moved from close to $50 \%$ withdrawal at $19.8 \mathrm{~cm}$ to $40 \mathrm{~cm}$, corresponding to a $100 \%$ withdrawal. The safety shims undergo a slight insertion to compensate, moving from $28.4 \mathrm{~cm}$ to $26.9 \mathrm{~cm}$ to maintain a close approximation of criticality. The estimates of the observable Cerenkov flux above the core in each coolant channel for the two states are subtracted from one another. For each channel, the difference is calculated using equation 12 :

$$
\text { Perturbed flux }- \text { baseline flux }=\text { absolute difference. }
$$

Plotting the difference for each channel allows the effects of the perturbation to be seen more easily. In Figure 11, the absolute difference plot for the case with the regulating rod withdrawn can be seen. The removal of the regulating rod and slight insertion of the shims causes a small decrease in the power in the lower left portion of the plot, as seen by the light blue region. The red region near the regulating rod corresponds to an increase in the Cerenkov observed in that area, caused by an increase in the neutron flux and fission rate, due to the removal of an absorber. The effect is almost invisible in this plot, because the absolute change in Cerenkov is being plotted, and the primary location of the change is at the periphery of the core, where the flux is lowest. A second difference plot, showing the relative difference of each channel is also produced, using equation 13:

$$
\frac{\text { Perturbed flux-baseline flux }}{\text { baseline flux }}=\text { relative difference } .
$$

The relative difference plot can be seen in Figure 12. Analyzing the relative difference makes the change in Cerenkov production near the regulating rod very apparent. The four coolant channels in the top right corner border the regulating rod, so as 
expected, that is where the change is the most significant. A smaller decrease in the rest of the top right quadrant of the diagram is also evident due to the depression in the neutron flux.

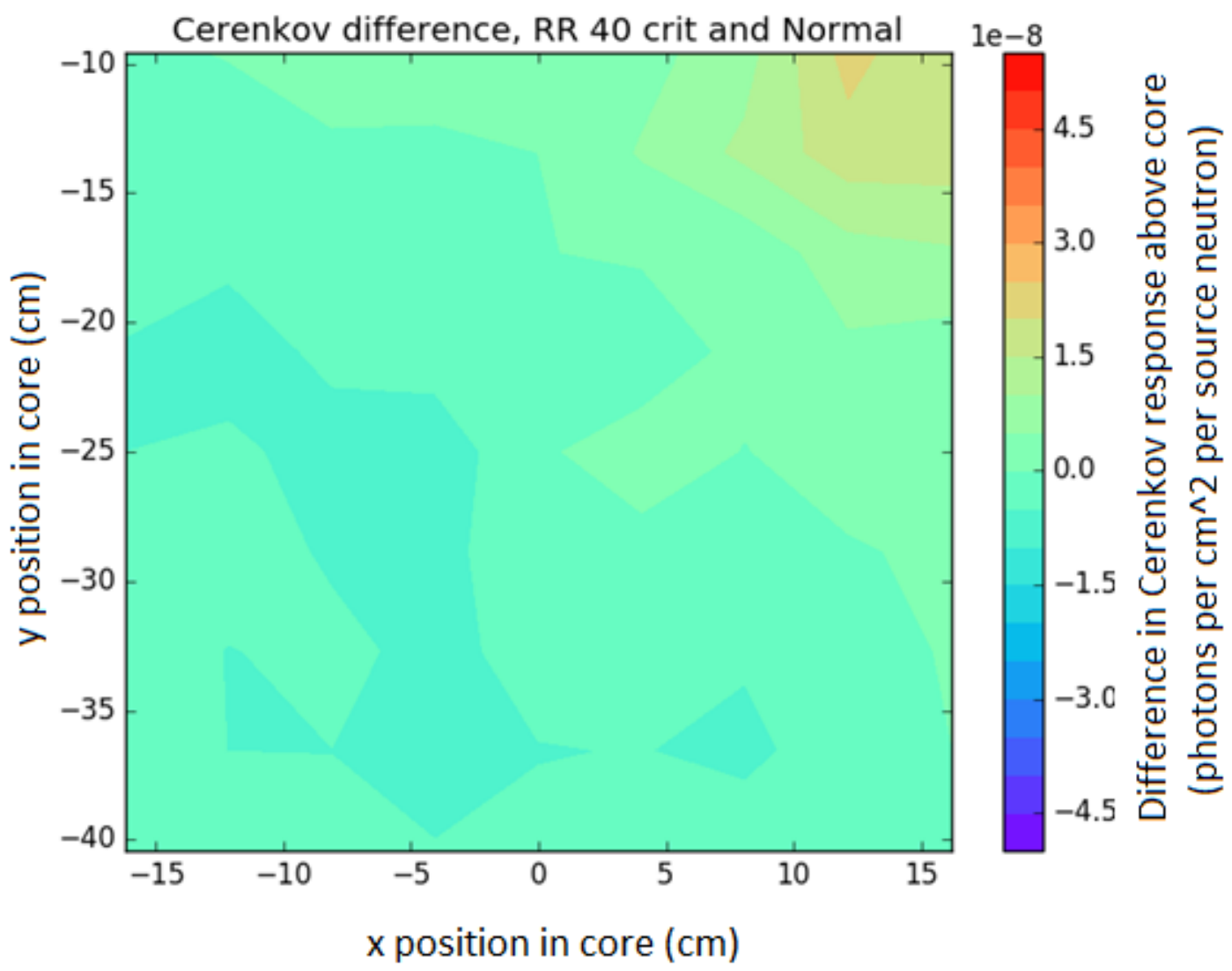

Figure 11 Absolute difference in Cerenkov above the core comparing the reactor with the regulating rod fully withdrawn to the baseline case with the regulating rod close to $50 \%$ withdrawal. 


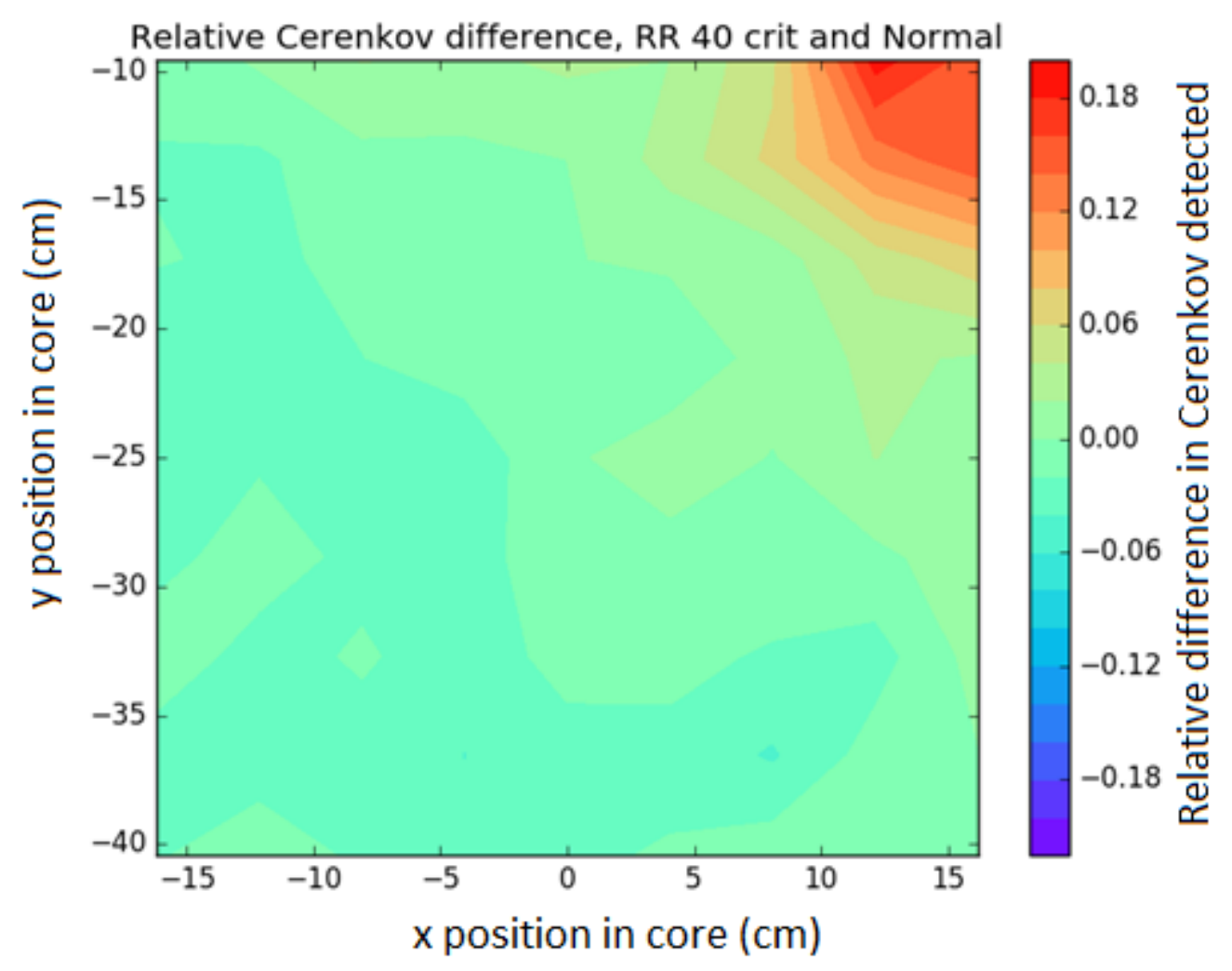

Figure 12 Relative difference in Cerenkov above the core comparing the reactor with the regulating rod fully withdrawn to the baseline case with the regulating rod close to $50 \%$ withdrawal

The second comparison case modeled has the transient control rod near the middle of the reactor inserted to $20 \mathrm{~cm}$, corresponding to a $50 \%$ withdrawal from the reactor, compared to the base state with a $100 \%$ withdrawn transient rod. The negative reactivity introduced by the transient rod insertion is counterbalanced by the almost complete removal of the shims to $36.8 \mathrm{~cm}$, or $92 \%$ withdrawal. Any further insertion of the transient rod would not allow the reactor to maintain criticality with the same power level and fuel temperature. The transient rod is kept fully withdrawn during normal steady state reactor operation, and is primarily used when the reactor is operating in pulse mode. A partial insertion of the transient rod when the reactor is at full power is an unusual situation, but 
it is physically possible and provides some interesting flux shifts. A plot of the absolute difference in the Cerenkov radiation above the core caused by inserting the transient rod can be seen in Figure 13, and the relative difference can be seen in Figure 14. A large flux decrease can be seen in the middle of the core. The point where the magnitude of the drop is greatest corresponds to the coolant channel above and to the left of the transient rod in the reactor map, with the other three channels bordering the rod making up the rest of the bluest region on the plot. This depression in the flux is to be expected, and the ease with which the difference can be noticed in the estimated Cerenkov observations shows potential for its use as an additional method for detecting power shifts.

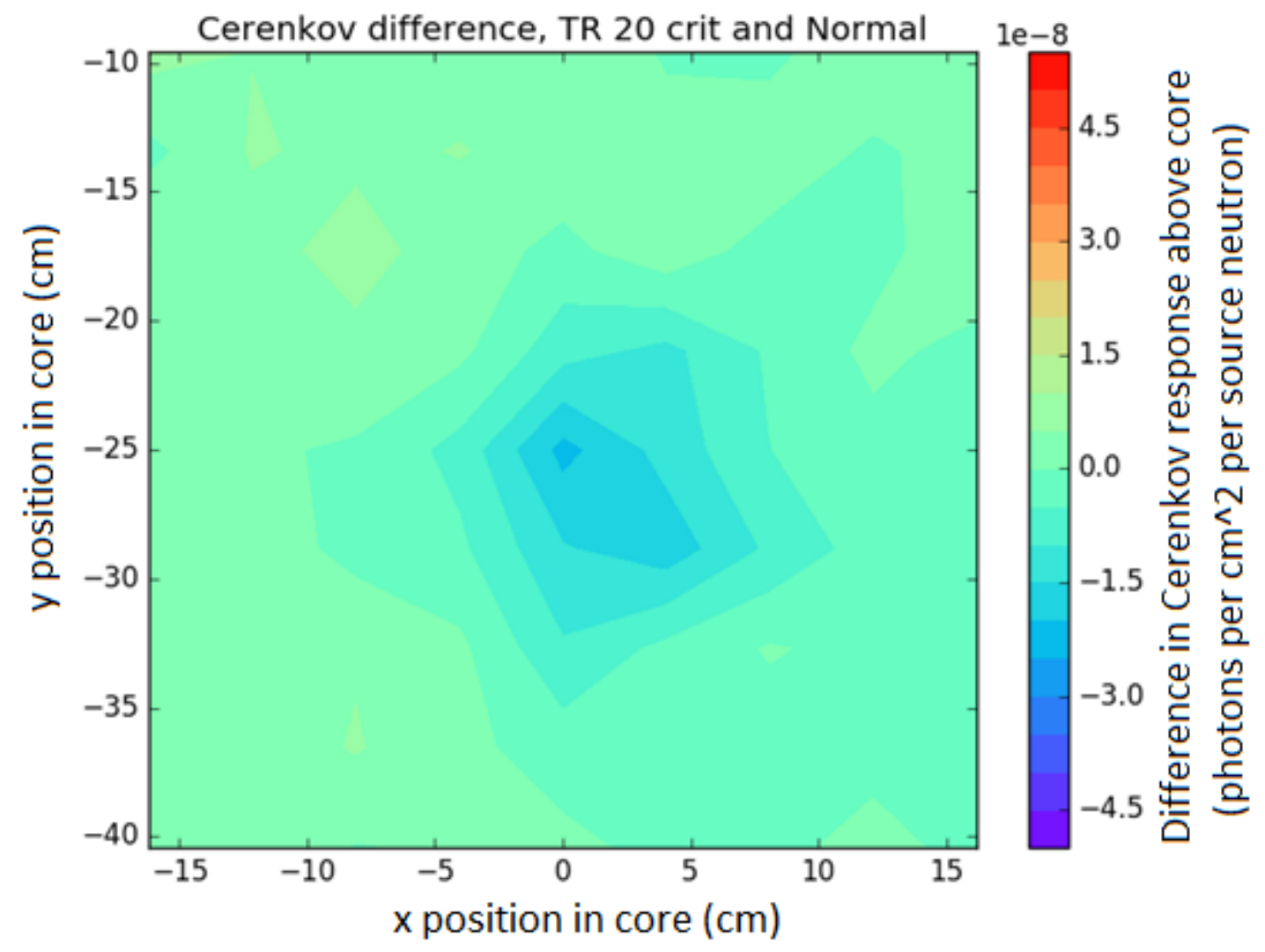

Figure 13 Absolute Cerenkov difference when inserting the transient rod to a position of $20 \mathrm{~cm}$. 


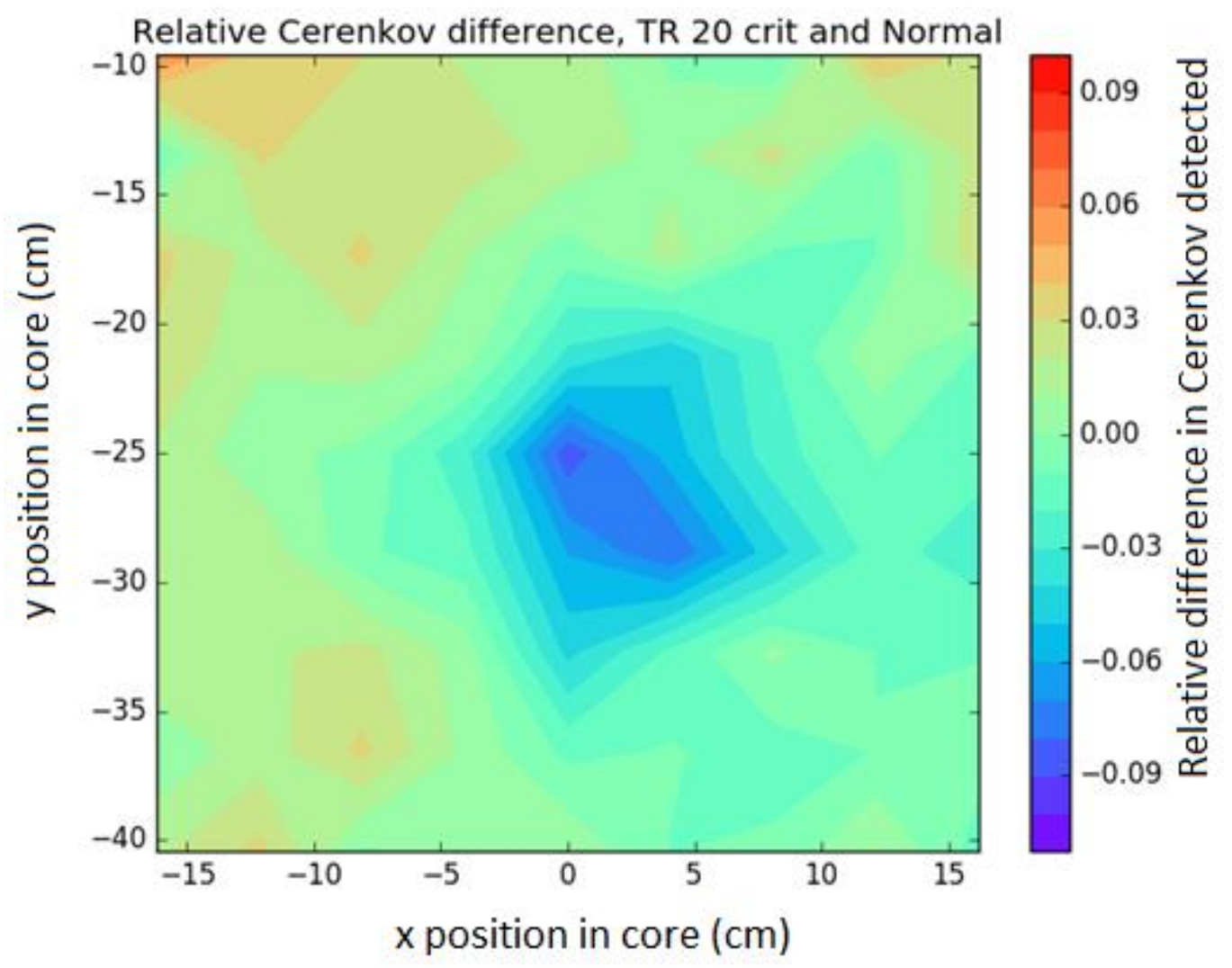

\section{Figure 14 Relative Cerenkov difference when inserting the transient rod to a position of $20 \mathrm{~cm}$}

The third state change analyzed is the movement of the reactor next to the graphite coupler box. The $+y$ direction face of the reactor is normally some distance away from the graphite box, such that the water between them is the only thing meaningfully affecting the reactor physics. The reactor can be moved within the pool to come very close to the coupler box so that neutrons from the core can diffuse into the coupler and down the neutron beam ports that are attached to it to be used for neutron radiography or other experiments. Changing the boundary condition of that face of the core from water to graphite has a large effect on the reactivity and the flux distribution in the core, because 
the graphite absorbs less neutrons and acts as a better reflector than open water. To balance the reactivity insertion, the safety shims are inserted to $17 \mathrm{~cm}$, compared to the baseline position of $28.4 \mathrm{~cm}$. A VISED picture of the core next to the coupler box can be seen in Figure 15. A plot of the total Cerenkov flux with the reactor next to the coupler is shown in Figure 16. The peak of the observable Cerenkov flux is in the vicinity of $y=-15$ to -20 , compared to the baseline Cerenkov map that has its peak in the -20 to -25 region. This corresponds to a shift of about one pin unit cell. This $+y$ shift in the flux profile is present throughout the rest of the core as well, such that the bottom right portion of the plot has less Cerenkov flux than the minimum bound used for the plots.

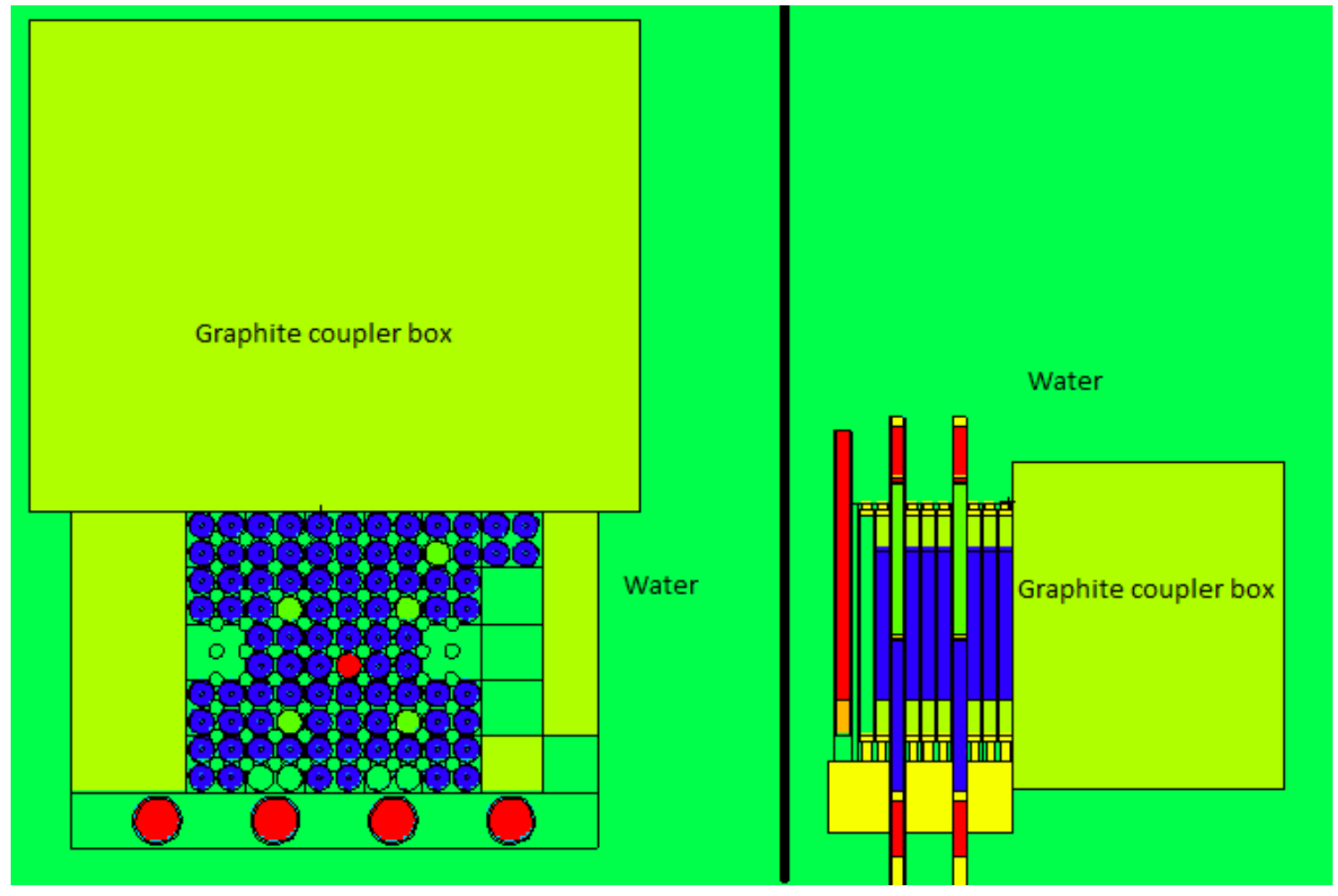

Figure 15 Reactor core when moved next to coupler box, showing XY (left) and YZ (right) views of the core 


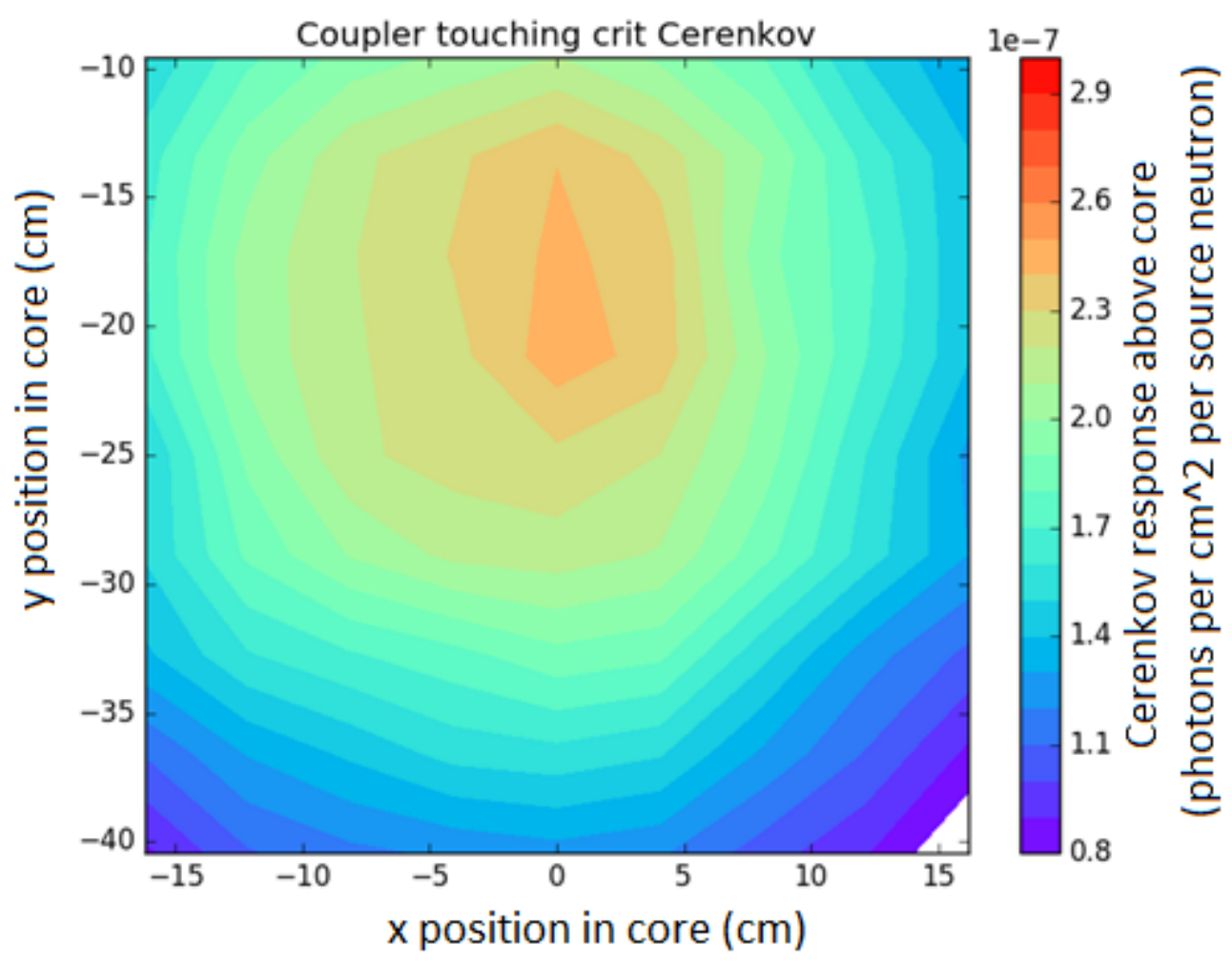

Figure 16 Map of total Cerenkov above core with graphite coupler adjacent to core.

The absolute and relative comparative plots for the movement of the core towards the coupler box can be seen below in Figure 17 and Figure 18 respectively. There is a very large change in the Cerenkov production at the top of the plot, near the interface with the coupler box. Due to the low initial flux in this region, some points in relative difference plot exceeds the $20 \%$ bound on the scaling used for relative difference plots. The blue spots in the bottom portion of the plots and the small blue bumps in the line across the $20 \mathrm{~cm}$ region correspond to the channels near the safety shims, where the local flux depression is more pronounced. The general gradient from the top to the bottom of the plot shows the lateral tilt of the flux across the whole core; this result has potential for being more directly quantified using the spatial derivative of the flux either at specific 
points, or averaged through the whole core. The usage of many channels across the core can make overall lateral tilts more apparent and reduce the reliance on individual data points. The detection of axial flux tilts is discussed in the three dimensional extrapolation section (chapter 3).

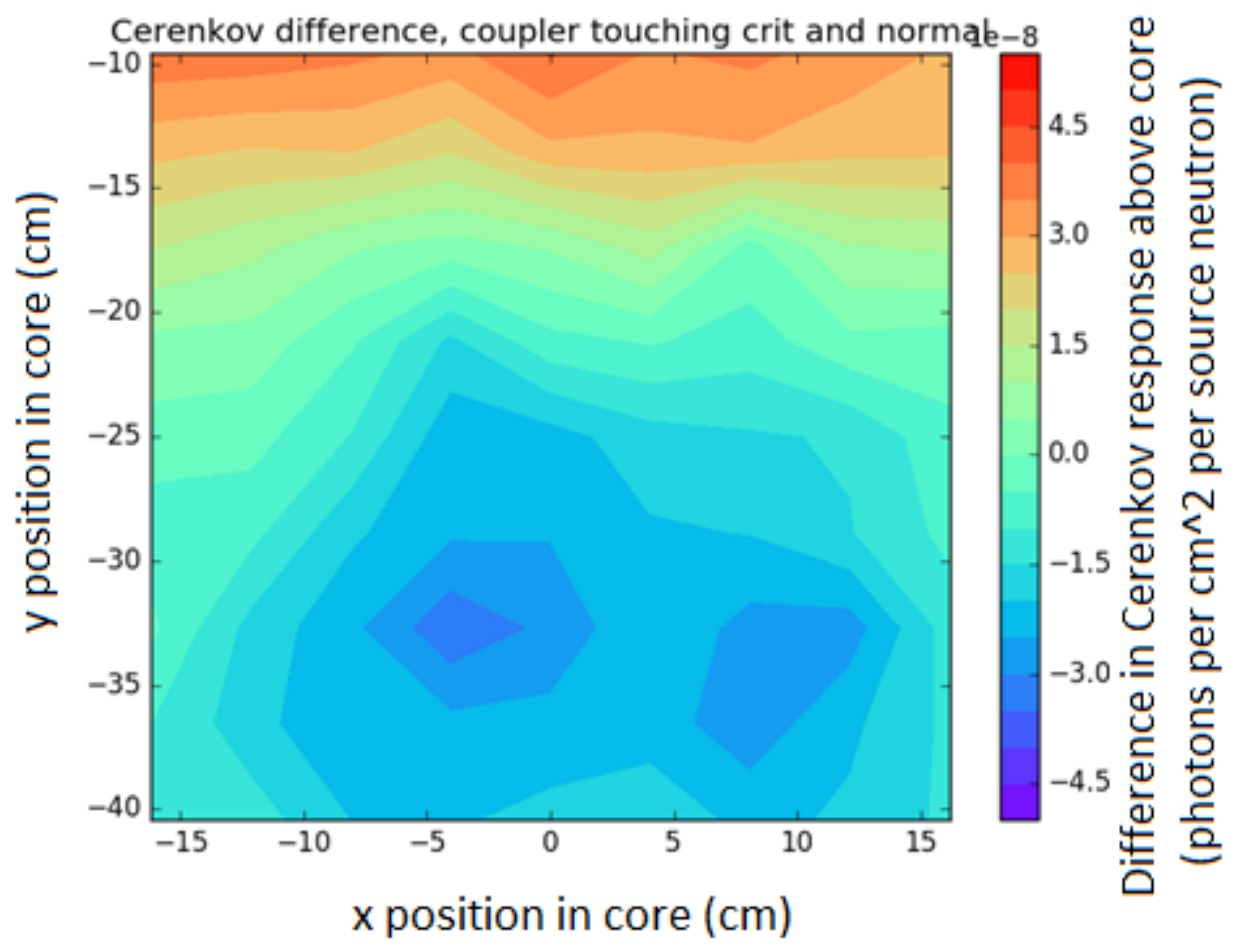

Figure 17 Absolute Cerenkov difference when moving the core next to the graphite coupler box. 


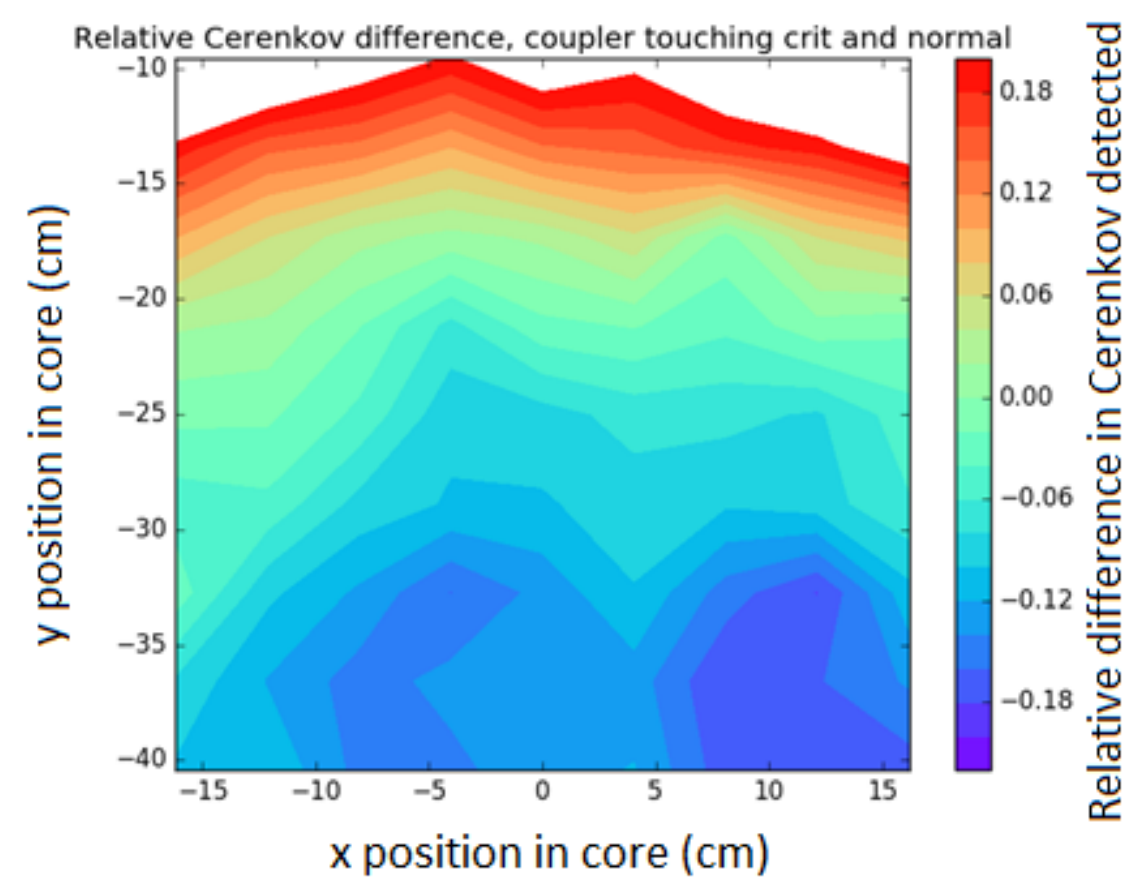

Figure 18 Relative Cerenkov difference when moving the core near the coupler box. The white region at the top corresponds to a greater than $20 \%$ flux increase.

A similar though less pronounced type of whole core tilt can occur in some large thermal reactors when there is unequal fission product buildup and burn off, causing a phenomenon referred to as Xenon oscillations. Xenon oscillations are a positive feedback mechanism where a region of the reactor that has an increase in the flux starts to burn the highly absorptive indirect fission product Xenon-135 out faster than it is being produced from decay. This initially causes a further increase in the flux in that region, until the increased fission rate and production of Xenon 135 through the decay of Iodine-135 catches up, after which the Xenon levels in the fuel will increase beyond equilibrium, causing a decrease in the flux. These oscillations can lead to instabilities that require control actions and possibly early reactor shutdowns, a problem that has been studied 
extensively for large PWRs [49] [50] [51] [52] and other large thermal reactors [53]. Early, accurate, detailed detection of flux shifts can allow action to be taken to mitigate such issues in a more effective manner. 


\section{THREE DIMENSIONAL CERENKOV PRODUCTION EXTRAPOLATION}

\subsection{Use of multiple viewpoints and shadowing for 3D extrapolation}

All previous Cerenkov estimates are for the light detected directly above the coolant channel, with no shadowing caused by the channel. As the number of detectors to be used is not set, the approximation could be valid or very close to accurate if horizontal distance between the coolant channel's center and the detection point is small, or corrections could be made as mentioned previously. The information that can be gained from a detector above a coolant channel is limited to the total amount of Cerenkov coming from the channel, with no axial determination, restricting the information to a 2D map of the core.

To include axial dependence and gain 3D information using visual detectors above the core, a method that compares the Cerenkov flux in a single channel observed from different positions can be used. An observation position that is horizontally offset from the coolant channel's center will observe more of the light from the top of the channel and less of the light from the bottom, due to the fuel pins blocking the light from reaching the detector. The left side of Figure 19 shows how an offset viewpoint can have axial dependence for light detected from a channel, where the grey shaded region is the portion of the coolant channel that is "seen by" and contributing to the Cerenkov flux in the offset detector. For simplicity, the offset position analyzed is chosen such that exactly half of the total volume of the channel is visible; corresponding to a $7 \mathrm{~cm}$ offset $2 \mathrm{~m}$ above the core midplane. Also, an approximation is made that treats the coolant channels and fuel pins as rectangular prisms is used to make the shadowing effects simpler, shown in the right side 
of Figure 19. The square channel approximation is only used in determining the Cerenkov contributions for different axial segments of the coolant channel and does not otherwise affect the model or neutronics in any way.

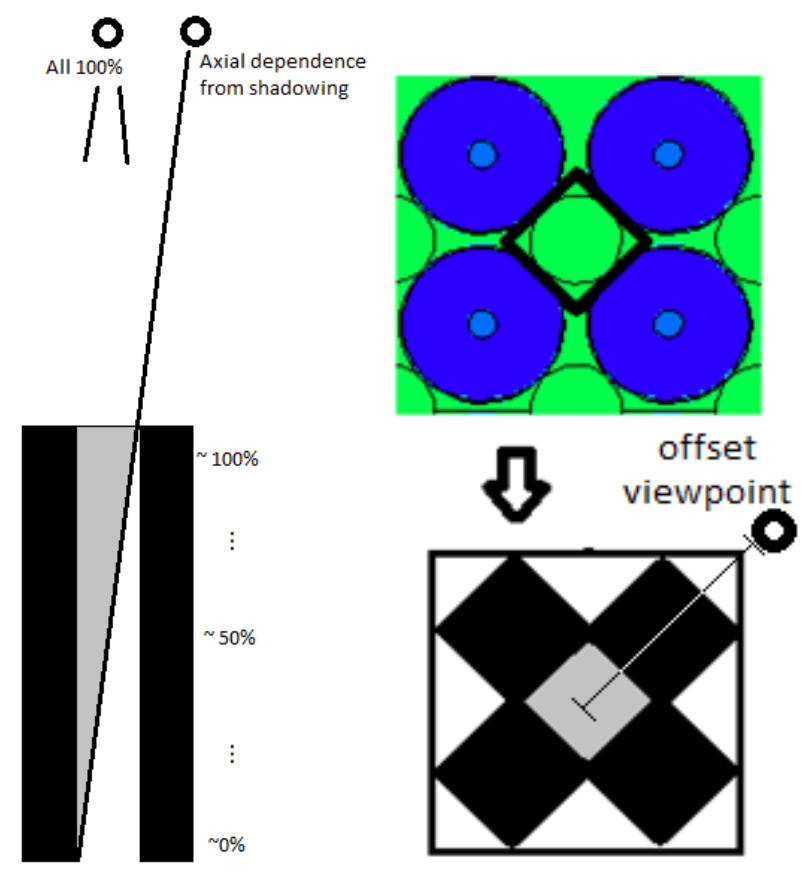

Figure 19 Coolant channel segment contribution to Cerenkov observed at an offset from the channel centerline (left), where the shaded region is the only portion contributing to the signal at the offset location. Also, (right) the square coolant channel shadowing approximation, showing how the coolant channel and pins are approximated as square with an offset viewpoint perpendicular to the flat face of the square channel to simplify the geometry.

The amount of light detected at the offset position is calculated using the same python script and method as is used for the detectors directly above the channel, except that there are modifications to the contributions from each axial segment. The script can be seen in Appendix C. As before, the total is calculated by summing the contributions of the electron tally bins using the segment and energy specific electron to Cerenkov 
correlation factors. The modification is that for the offset viewpoint, each segment is also multiplied by its contribution factor, a number between 0 and 1, to account for shadowing. This is a modification to equation 10 and can be seen in equation 14:

$$
\Psi_{\text {offset }}=\sum_{n=1}^{E \text { bins }} \sum_{m=1}^{\text {Segments }} \Phi_{\text {Electron }}\left(z_{m}, E_{n}\right) \sum_{\text {Response }}\left(z_{m}, E_{n}\right) * \chi_{\text {contribtion }}\left(z_{m}\right) .
$$

The contribution factor, $\chi_{\text {contribution }}$ of a given segment to the offset detector's total is calculated using equation 15 :

$$
\text { Segment contribution factor }=\chi_{\text {contribution }}=\frac{\text { volume of segment visible }}{\text { total volume of segment }} .
$$

The square channel approximation makes this a fairly simple and consistent calculation to perform. The contributions for four of the 16 segments can be seen in Figure 20. The top segment, bottom segment and the two middle segments are shown along with the upper and lower bounds of the segment are denoted by red lines and text. The grey shaded area shows the region that is contributing to the offset viewpoint's Cerenkov flux. The top and bottom segments are slightly smaller than the other segments.

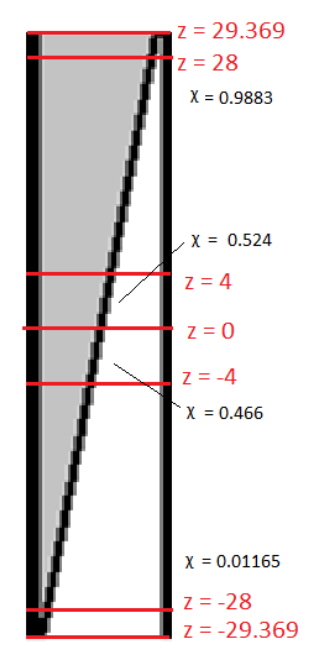

Figure 20 Contribution factors for the top, two middle and bottom segments in the coolant channel. 
This model uses the aforementioned idealized square channel and an exact offset viewpoint that perfectly divides the square prism shaped coolant channel along a diagonal plane. In a real system, the segment contribution factors would be different for each channel and the offset viewpoints would be limited to the locations that have detectors. One matter of efficiency that can be implemented is to use of the detector directly above one coolant channel as the offset detector above another, depending on the type, number and spacing of the detectors and fuel rods. Also, as mentioned in the end of the 2D Cerenkov mapping section, the system could use a single camera type detector as the detector positioned close to "directly above" many separate channels, correcting for the small shadowing effects. This could allow a close to direct viewpoint and a more substantially offset viewpoint to be used for every channel using a relatively small number of detectors by taking advantage of the fact that a detector above one channel is at an offset from another channel. Determining the offset factors involved for many different viewpoint offsets and more realistic channel shapes would require somewhat rigorous geometric calculations and is not a part of this research effort, as its focus is as a proof of concept, rather than a finalized design for production and usage.

One other advantage to the method of using contribution factors to determine the observed Cerenkov flux at an offset position is that the calculations can be done without requiring MCNP to be run again. The transport of photons and electrons is computationally expensive, so avoiding having to run additional input files improves the rate that progress can be made. 


\subsection{Axial Cerenkov tilt determination}

The primary method of extracting axial information from the comparison of different Cerenkov detection points above the core is the calculation of an axial flux tilt for a coolant channel. The ratio of the offset Cerenkov flux to the flux directly above the channel provides information about whether the flux is shifted towards the top or bottom of the channel.

The segments near the top of the channel already contribute slightly more to the total Cerenkov above the core because they are closer to the tallying plane and thus have less loss due to the exponential attenuation and the $1 / \mathrm{r}^{2}$ light spreading effect. For this reason, even if the flux was perfectly symmetric about the axial centerline, the offset ratio will not be exactly 0.5 , but instead will be some value slightly above 0.5 . To determine what the symmetric baseline axial tilt is, the model with 4 pins in an infinitely reflected lattice is used. This model has no control rods or other perturbations that will shift the flux either up or down, so the neutron population should be very close to symmetric in the axial

direction. In the 4 pin reflected model the Cerenkov flux directly above the channel was 5.462E-6 and the offset flux was 2.866E-6. Using equation 16, the resulting flux tilt ratio is 0.5248 . The flux tilt value of 0.5248 will therefore be used as a zero actual tilt baseline for the flux tilt in the whole-core models. This is used in equation 17 to obtain the absolute flux tilt for any channel, such that an axially symmetric flux has zero tilt, as seen here:

$$
\begin{gathered}
\text { Axial offset ratio }=\text { flux tilt ratio }=\frac{\text { Cerenkov detection at of fset position }}{\text { Cerenkov directly above channel }}, \\
\text { absolute tilt }=\text { flux tilt ratio }- \text { symmetric tilt ratio } .
\end{gathered}
$$


It is expected that most if not all of the absolute flux tilts will be negative (the ratio is below 0.5248), because of the presence of absorbers in the top portion of the core from the various control rods. If the fuel composition was heterogeneously burned, it could result in positive axial flux tilts, but this is not done in the model used for tracking electrons. Most of the analysis of flux tilts will focus on changes in the tilt when the state of the reactor is altered, so positive changes in the tilts will occur.

For the flux tilt analysis of reactor state changes, the normal critical case is again used as a baseline. Again, the regulating rod is at $19.8 \mathrm{~cm}$, the shims are at $28.4 \mathrm{~cm}$, the transient rod is fully withdrawn, and the coupler box is not close to the core. A plot showing the flux tilt ratio normalized to the infinite lattice symmetric flux baseline for each coolant channel can be seen in Figure 21. This plot is a plot of the flux tilt ratio except that the values has been shifted downward by 0.5248 . In the plot of the flux tilt for the reactor in the normal state, every coolant channel has a negative absolute tilt as expected. The additional downward shift from local changes caused by each of the 4 safety shims can be seen in the 4 bluish depressions around the center of the plot. The partially inserted regulating rod also causes a notable downward flux tilt seen in the top right corner of the plot. The rest of the periphery of the reactor has a less pronounced tilt due to the lack of any nearby elements that are axially asymmetric. 


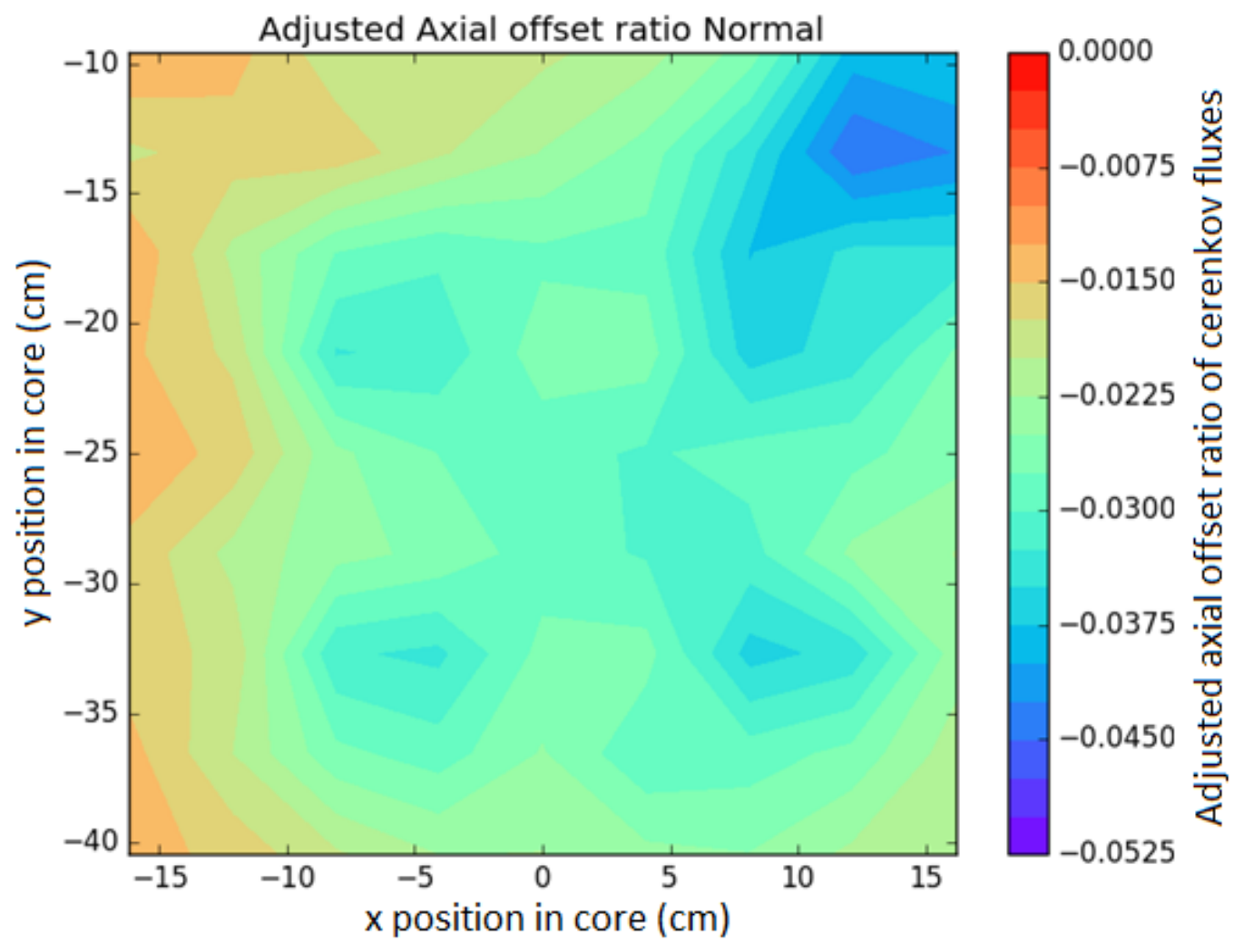

Figure 21 absolute tilt normalized to symmetric channel for each of the 81 coolant channels analyzed. The reactor is in the normal critical state.

The normal state flux tilts are used as a baseline to determine tilt changes using equation 18:

$$
\text { change in tilt }=\text { perturbed tilt ratio }- \text { baseline tilt ratio } .
$$

Changes do not need to be normalized to the symmetric case, and the normalization would cancel itself out anyways due to subtraction.

\subsection{State change analysis of axial tilts}

In this section, various reactor state changes are modeled to show how the axial Cerenkov flux tilt will respond to the change. The goal is to demonstrate the ability of the 
Cerenkov flux tilt measurements to detect changes in the axial fission rate density and neutron population. The state changes are all compared to the normal critical state mentioned above. The comparison in the tilt in each channel is done using equation 18 . The color scale used for plotting the tilt difference is changed from the rainbow scale used previously to a blue-white-red scale so that zero change is represented by white, and a darker red or blue represents a larger downward or upward flux shift.

The first perturbed state analyzed is the withdrawal of the regulating rod. The regulating rod is completely withdrawn from its initial position at $19.8 \mathrm{~cm}$ to $40 \mathrm{~cm}$. The safety shims are slightly inserted to compensate for the reactivity change. The regulating rod has a low reactivity worth, so the shims are only moved from 28.4 to $26.9 \mathrm{~cm}$. The flux tilt plot with the regulating rod withdrawn can be seen in Figure 22. There is no longer a large negative flux tilt in the top right corner because the regulating rod is not causing increased absorption near the top of the core. The four downward tilt peaks near the shims are still present. 


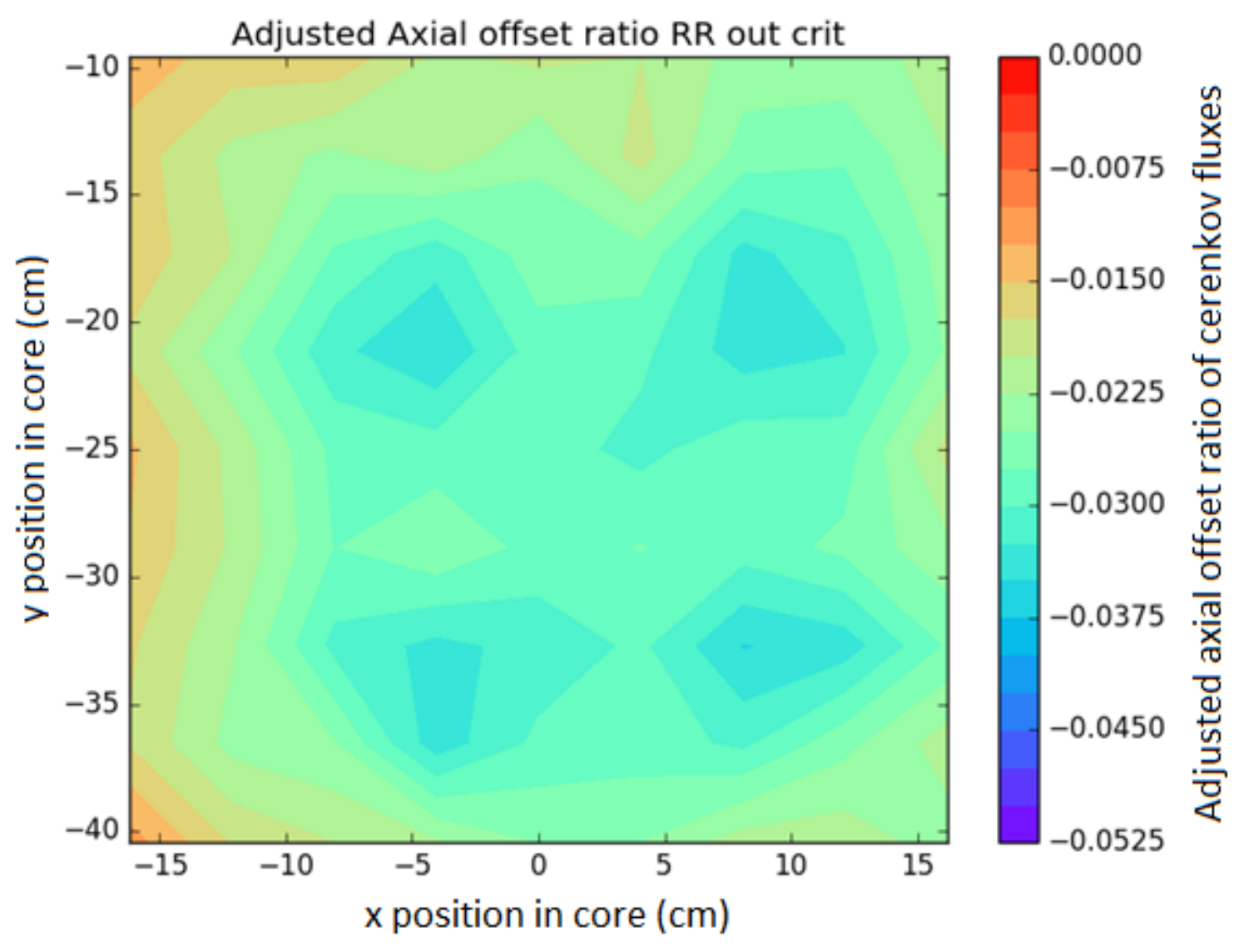

Figure 22 Flux tilt map with regulating rod fully withdrawn.

The difference plot that compares the flux tilts in with the regulating rod removed and with it in the baseline state can be seen in Figure 23. The large upward shift in the flux tilt in the top right clearly shows the effect of the motion of the regulating rod. The upward shift only affect the region around the rod. The changes in the rest of the plot are much smaller, the largest being approximately $1 / 3$ the magnitude of the shift near the regulating rod. Aside from the slight overall negative shift caused by the shims, the changes in the rest of the plot are due to uncertainty in the simulation. 


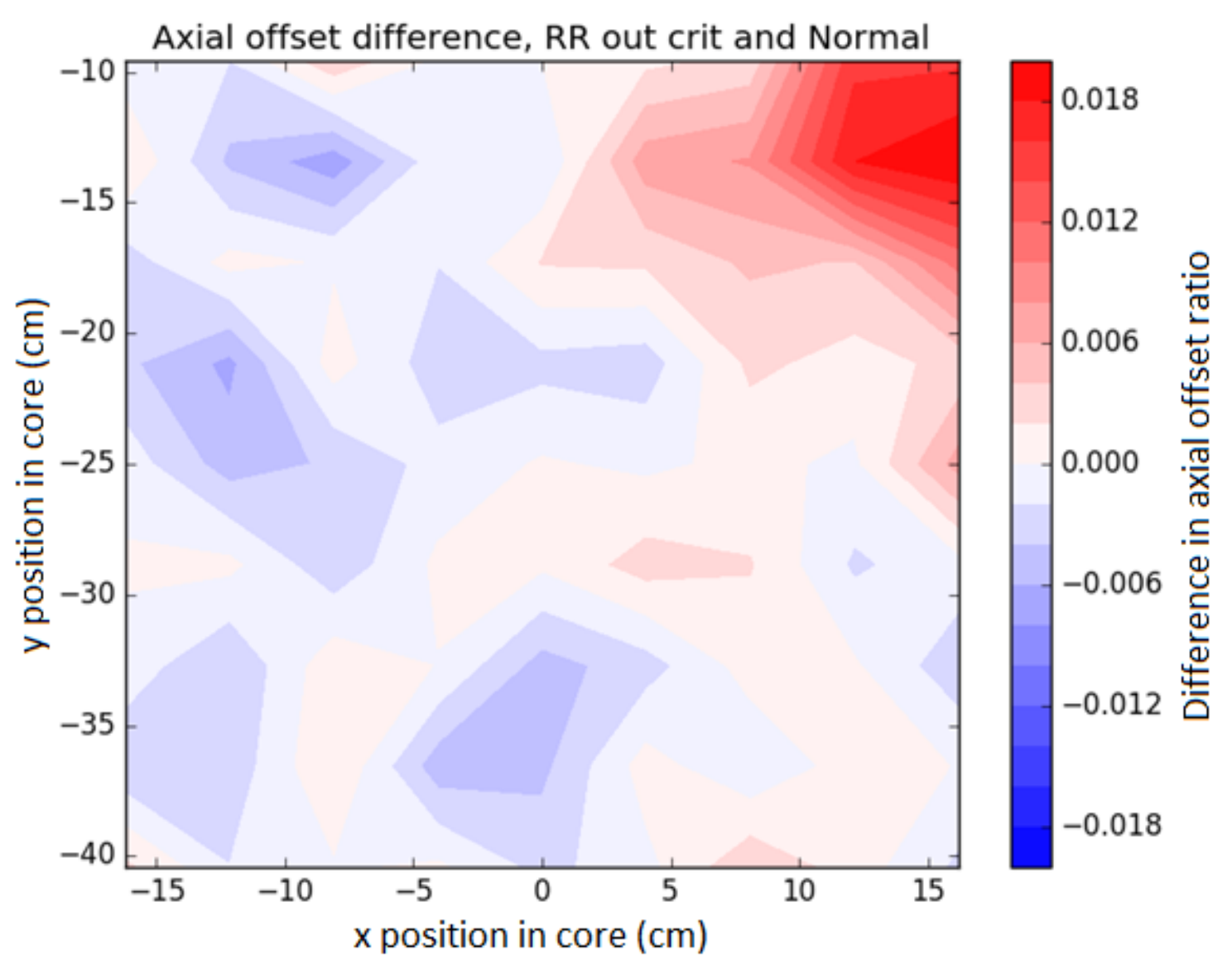

Figure 23 Change in flux tilt when removing the regulating rod and slightly inserting safety shims to maintain criticality.

A second perturbation involving the partial insertion of the transient rod is also modeled to observe how it changes the flux tilt. This rod movement involves inserting the transient rod to a position of $20 \mathrm{~cm}$, corresponding to $50 \%$ withdrawal. The safety shims are almost completely withdrawn, being moved from their normal state position of 28.4 $\mathrm{cm}$ to $36.8 \mathrm{~cm}$ to compensate for the reactivity loss when inserting the transient rod. The regulating rod remains at $19.8 \mathrm{~cm}$. With the shims withdrawn, they have relatively little effect on the flux tilt. The flux tilt plot for the reactor in this state can be seen in Figure 24. The distinctive low points in the tilt caused by the shims in the previous plots cannot 
be seen here, though there is now a depression in the center of the plot caused by the transient rod. The depression caused by the regulating rod is still present. The difference plot can be seen in Figure 25. Most of the difference plot is red, corresponding to an upwards shift in the flux through most of the reactor. This is most pronounced in some of the channels near the shims that have been withdrawn. There is a negative shift in the Cerenkov flux tilt in one channel next to the transient rod, but it would appear that the insertion of the transient rod has a much smaller effect on the overall axial flux tilt than the compensatory removal of the shims.

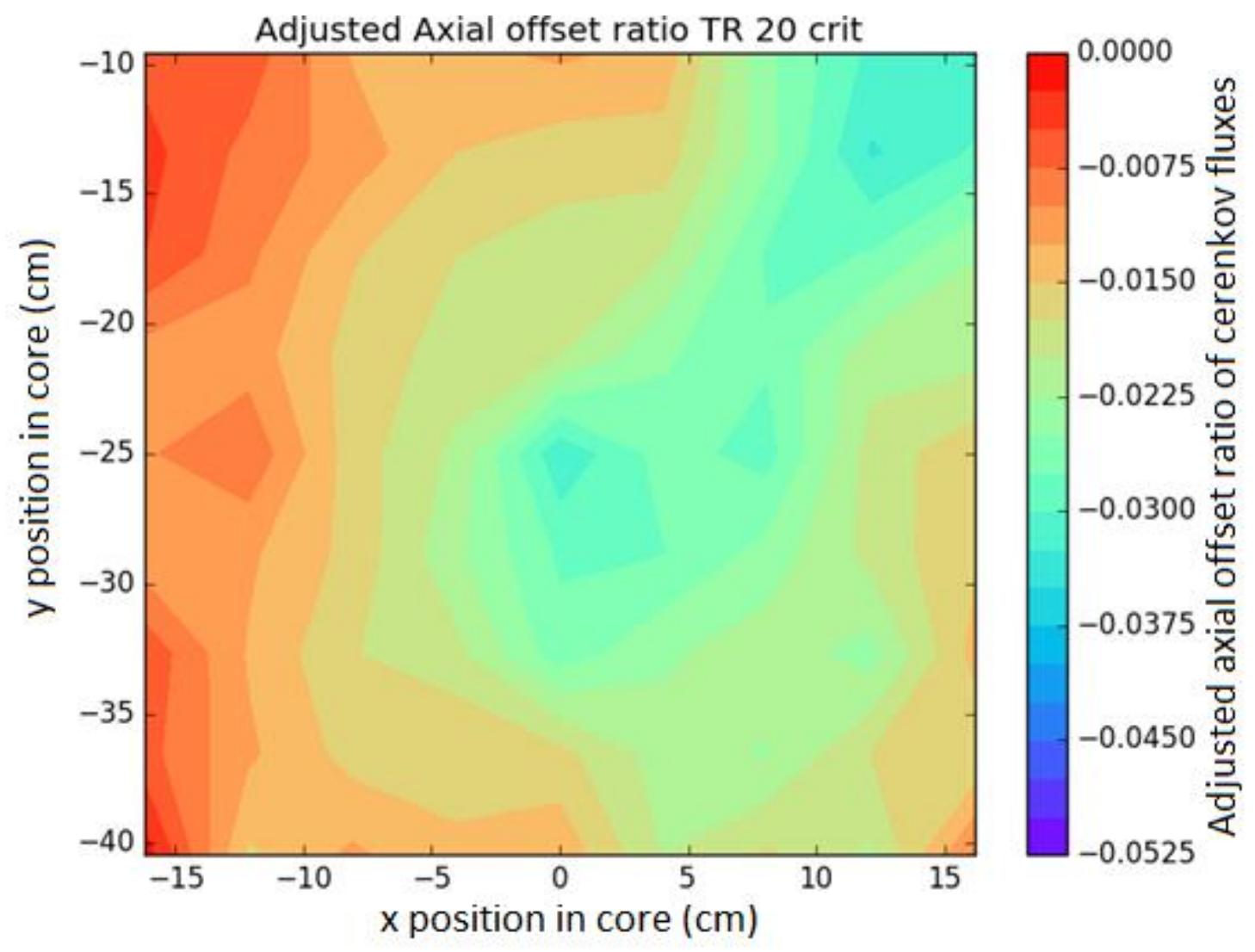

Figure 24 Flux tilt in each channel when the transient rod is halfway inserted and the shims moved further out to maintain criticality. 


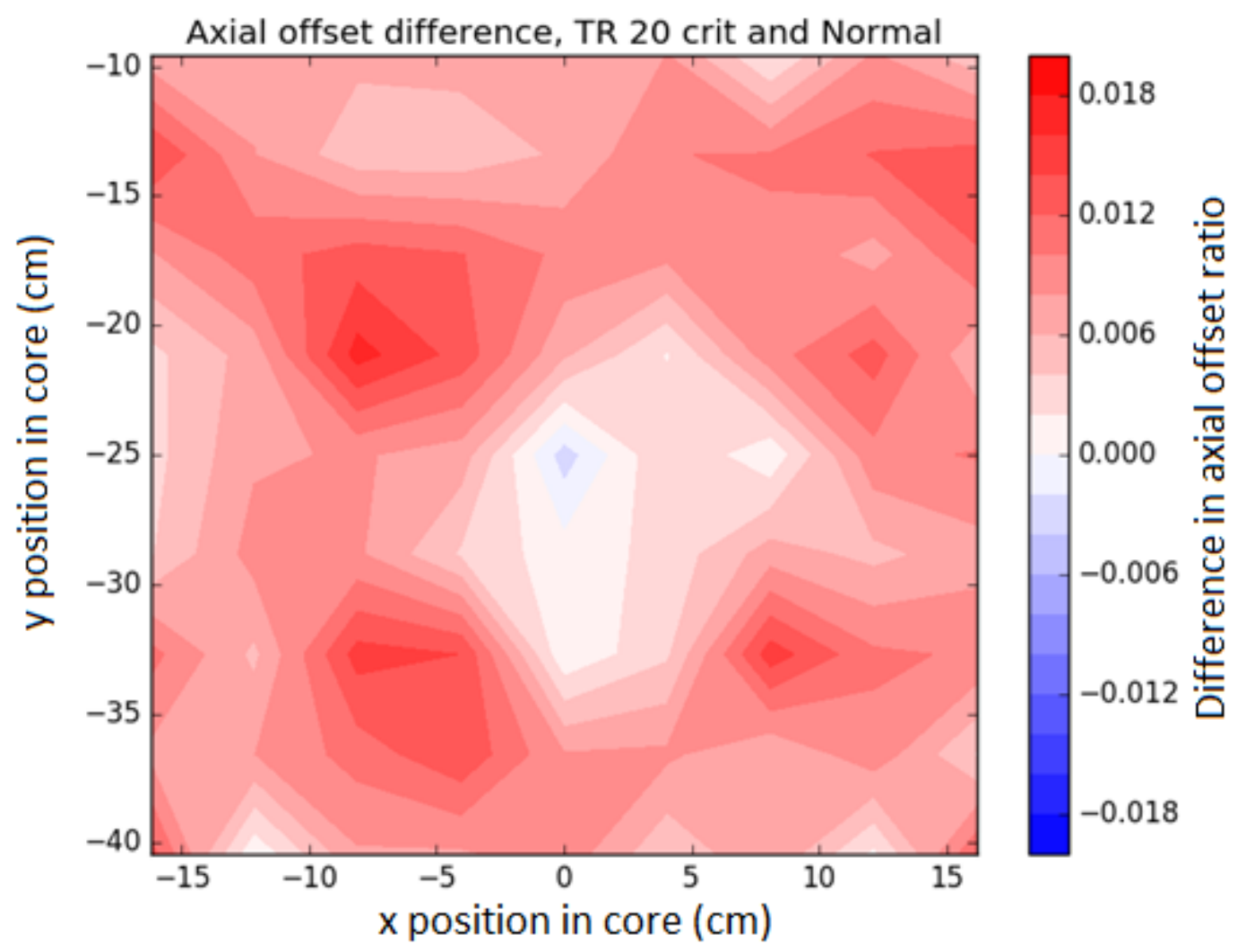

Figure 25 Change in the flux tilt when inserting the transient rod to $20 \mathrm{~cm}$ and withdrawing the shims accordingly.

The movement of the core such that it is next to the graphite coupler is also analyzed for its effects on the axial flux tilt. The coupler box is a much better neutron reflector than the open expanse of light water that is normally bordering that face of the core, so this motion causes a large reactivity insertion, requiring the safety shims to be inserted to a position of $17 \mathrm{~cm}$, compared to their initial position of $28.4 \mathrm{~cm}$. This creates a horizontal flux tilt that was mentioned in the 2D mapping section, and can be seen in the difference plot in Figure 17 and even more so in the relative difference plot in Figure 18, duplicated here as Figure 26 for easier reference. 


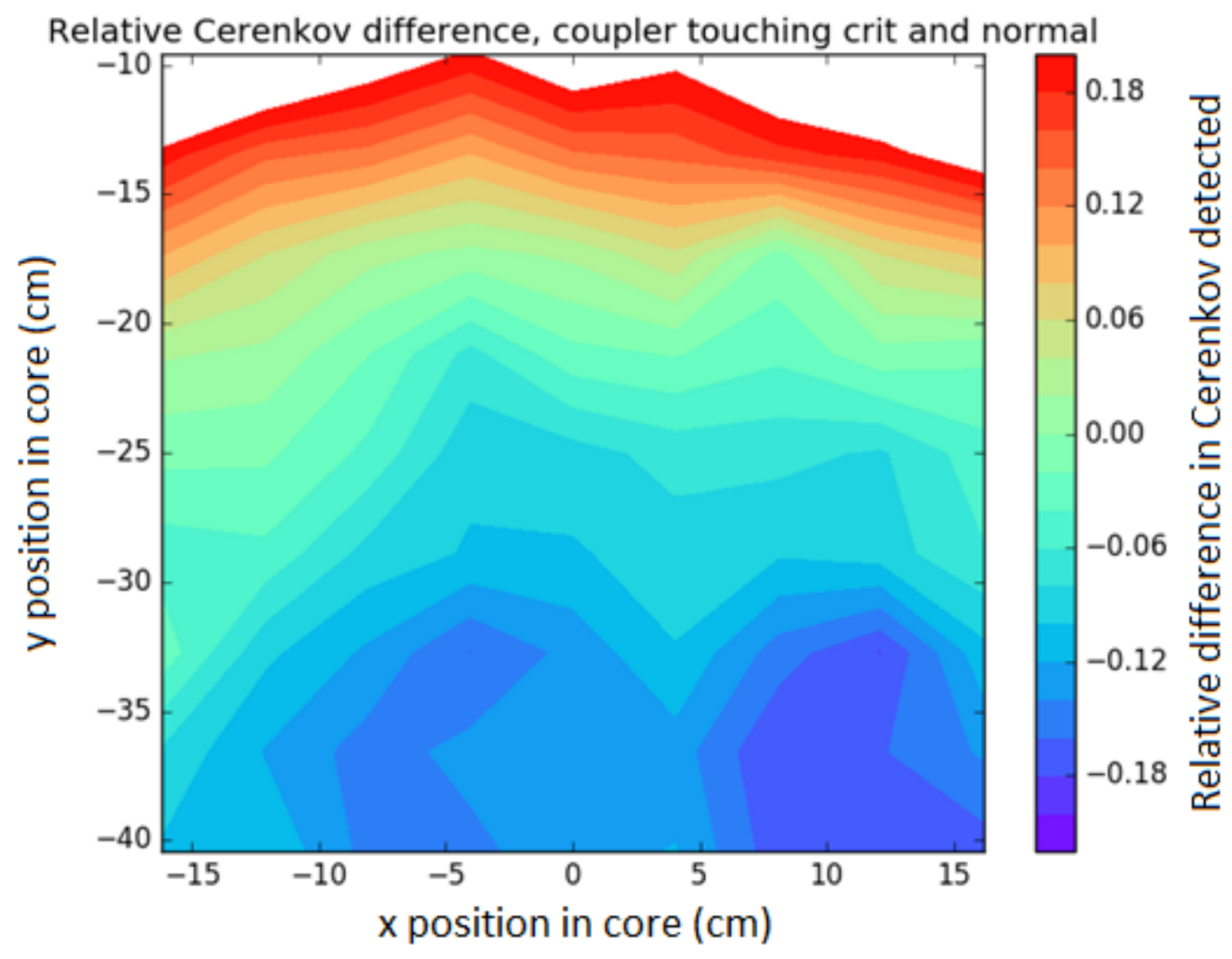

Figure 26 Relative change in the total Cerenkov flux directly above each channel when moving the coupler box next to the core and inserting shims to maintain criticality.

With such a large change in the total flux near the north portion of the core (the top of the plots), it is interesting to note that this is not at all shown in the flux tilt plots. The change in the axial offset is plotted in Figure 27. There is a slight downward shift in the flux tilt in the central region of the core where the effects of the shim insertions are present. The top of the plot shows little to no shift in the axial offset, in contrast to the more than $20 \%$ increase in the total flux seen above. This is an indication that the change in that region is more or less axially symmetrical. This is the case, because the change in the boundary condition uniformly affects the entire axial length of the fuel pins. The 
graphite block extends above and below the ends of the pins, so edge effects at the top and bottom are minimal.

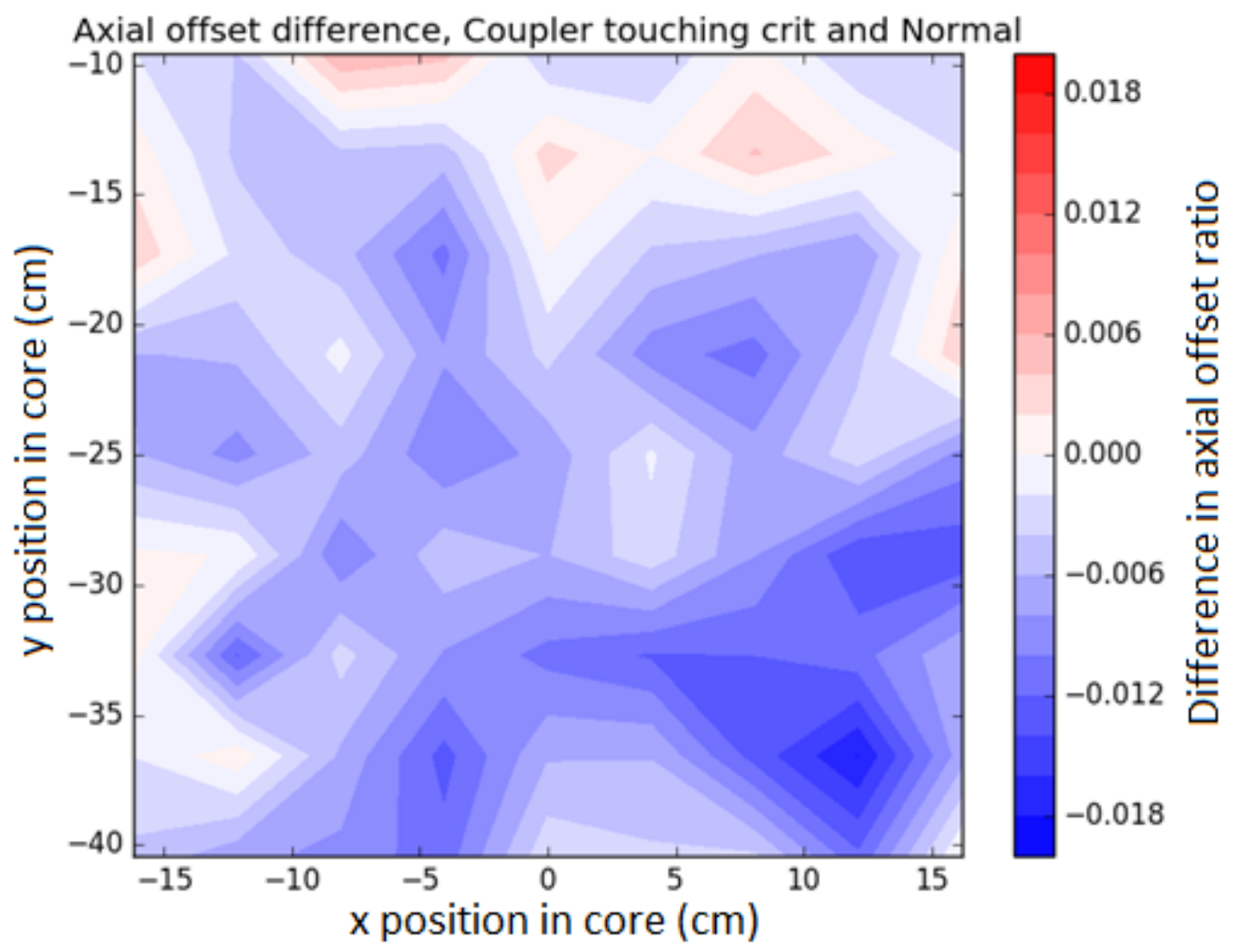

Figure 27 Change in flux tilt when moving the core next to the graphite coupler box.

Comparing the changes in the total flux and the changes in the axial tilt can determine whether the cause of the change is affecting the entire height of the core equally or if it is focused on the top or bottom half. Distinguishing between a uniform change and one that is not uniform but still axially symmetric; however, would require more offset viewpoints for each channel and more complex analysis methods than those employed in this research effort. 


\subsection{Coolant channel blockage analysis}

Another feature of the Cerenkov power monitoring system is the ability to quickly detect and pinpoint the location of some types of coolant channel blockages. Any obstruction of the middle or upper portion of a channel will cause an immediate change in the amount of Cerenkov light detected above the channel. The complete or partial blockage of a coolant channel will often have little to no immediate effect on the neutron or gamma flux in the core, and will only be detectable through conventional gamma or neutron measurements if the decrease in local cooling causes significant temperature feedback effects. Coolant temperature and flow measurements may be able to detect differences caused by a blockage, depending on where the block occurs relative to the flow monitors, but it is not a guarantee and the temperature measurements have a longer delay time. The example channel blockage analyzed here demonstrates the ease with which the visual information based power profile reconstruction method can locate a coolant channel obstruction.

To simulate a channel obstruction, the code used to post process the MCNP output deck was modified to include this ability. The code takes an input for the blocked coolant channel, at a specified height (which axial segment the block occurs in), and the portion of the channel that is blocked. It then calculates the blocked Cerenkov and blocked axial offset by reducing the contribution of all segments below the blockage to the total Cerenkov by the blockage factor. So for a full blockage at the midplane, all segments in the bottom half of the channel would contribute nothing to the total. For a $50 \%$ blockage, the segments below it would provide $50 \%$ of their normal contribution. 
A blockage is simulated in channel 4,4. This channel is between fuel pin 4,4, pin 4,5 , pin 5,4 and pin 5,5 . The blockage is at $\mathrm{z}=0 \mathrm{~cm}$, the core midplane, above axial segment 9 in the electron tally. It is a complete blockage, so the segments below it (9-16) have their Cerenkov contribution multiplied by zero. The resulting Cerenkov total is vastly decreased in channel 4,4 to $48.3 \%$ of its original value. The change in the Cerenkov map can be seen in Figure 28. A plot of the difference using the same scaling as previous 2D difference plots can be seen in Figure 29. The magnitude of the change is approximately 1.2e-7, which greatly exceeds the scale of the scale of the plot with a maximum magnitude of 5e-8. This would create a large signal in any monitoring system that could not be caused by any difference in the neutronics of the core, and thus can be clearly identified as either a blockage or a detector failure. A $52 \%$ blockage at the top of the fuel would cause the same effect in the total Cerenkov, so the offset information would also be needed to distinguish between the two different blockage scenarios. 


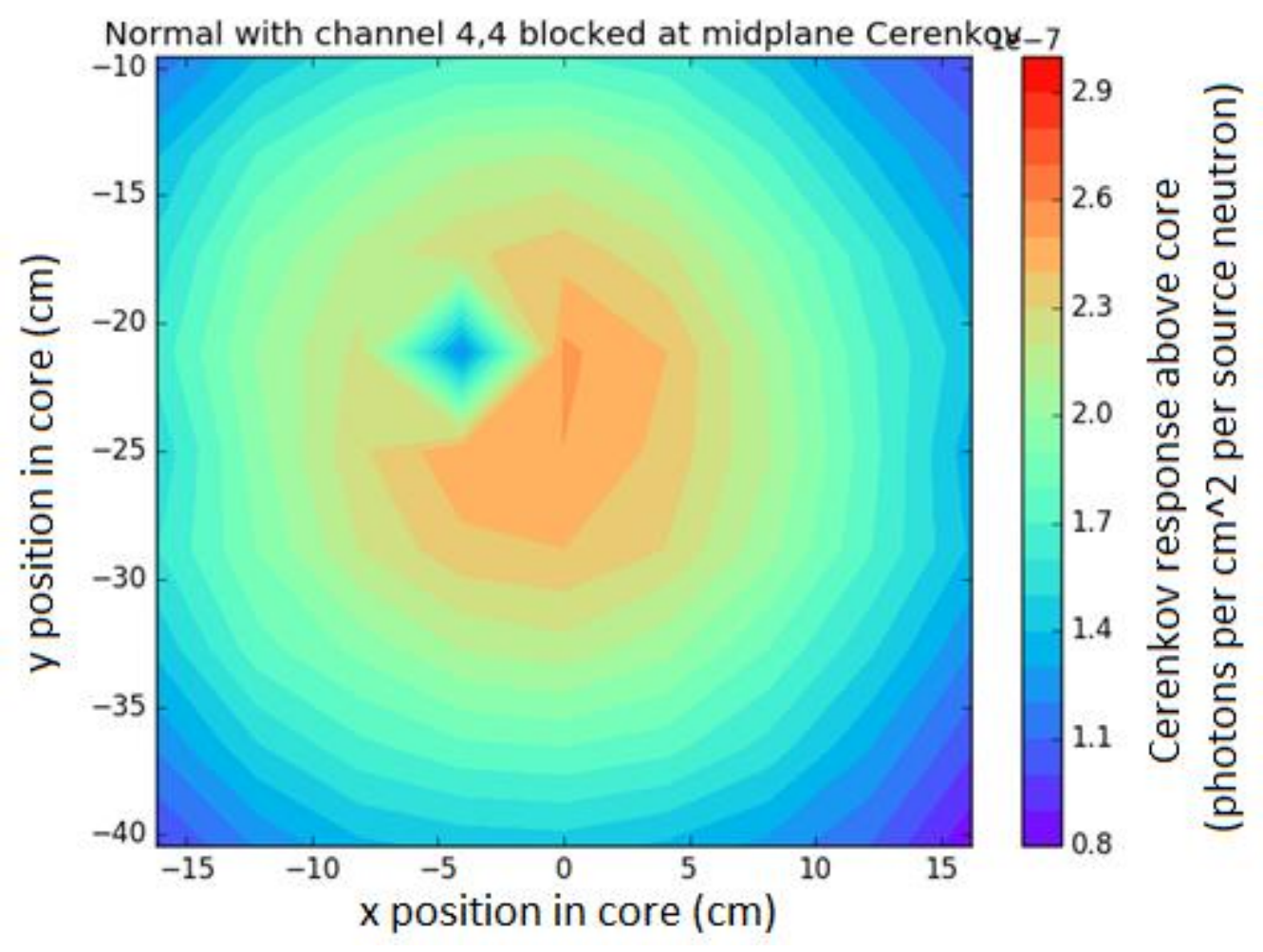

Figure 28 Total Cerenkov above core with channel 4,4 blocked $100 \%$ at midplane. 


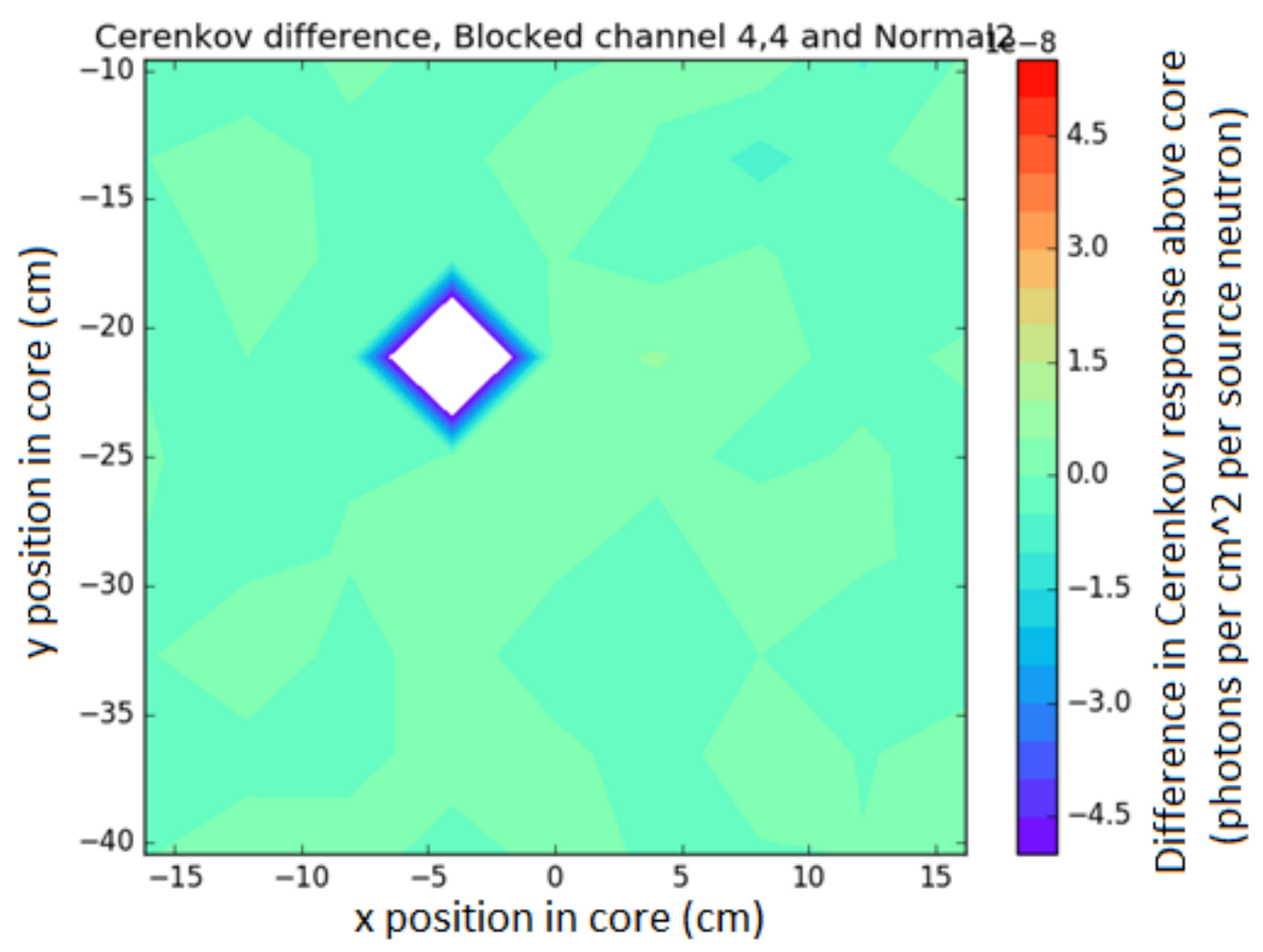

Figure 29 Cerenkov difference above core with a blockage in channel 4,4

The presence of a blockage also causes a large change in the axial Cerenkov tilt in the affected channel. In Figure 30, the axial offset for each channel can be seen with the blocked channel being the only one with a positive offset. A rescaled version of the plot can be seen in Figure 31. The offset in channel 4,4 is 0.682 , corresponding to an adjusted offset of 0.157 . This upwards tilt occurs because the Cerenkov detection directly above the channel was affected more than the offset location. The direct Cerenkov decreases from $2.42 \mathrm{e}-7$ to $1.17 \mathrm{e}-7$, for a $51.7 \%$ decrease, while the offset decreases from $1.20 \mathrm{e}-7$ to 7.99e- 8 , a $33.2 \%$ decrease. This information makes a detector failure in the detector above 
the channel a highly unlikely scenario, because two separate detectors are experiencing a major change.

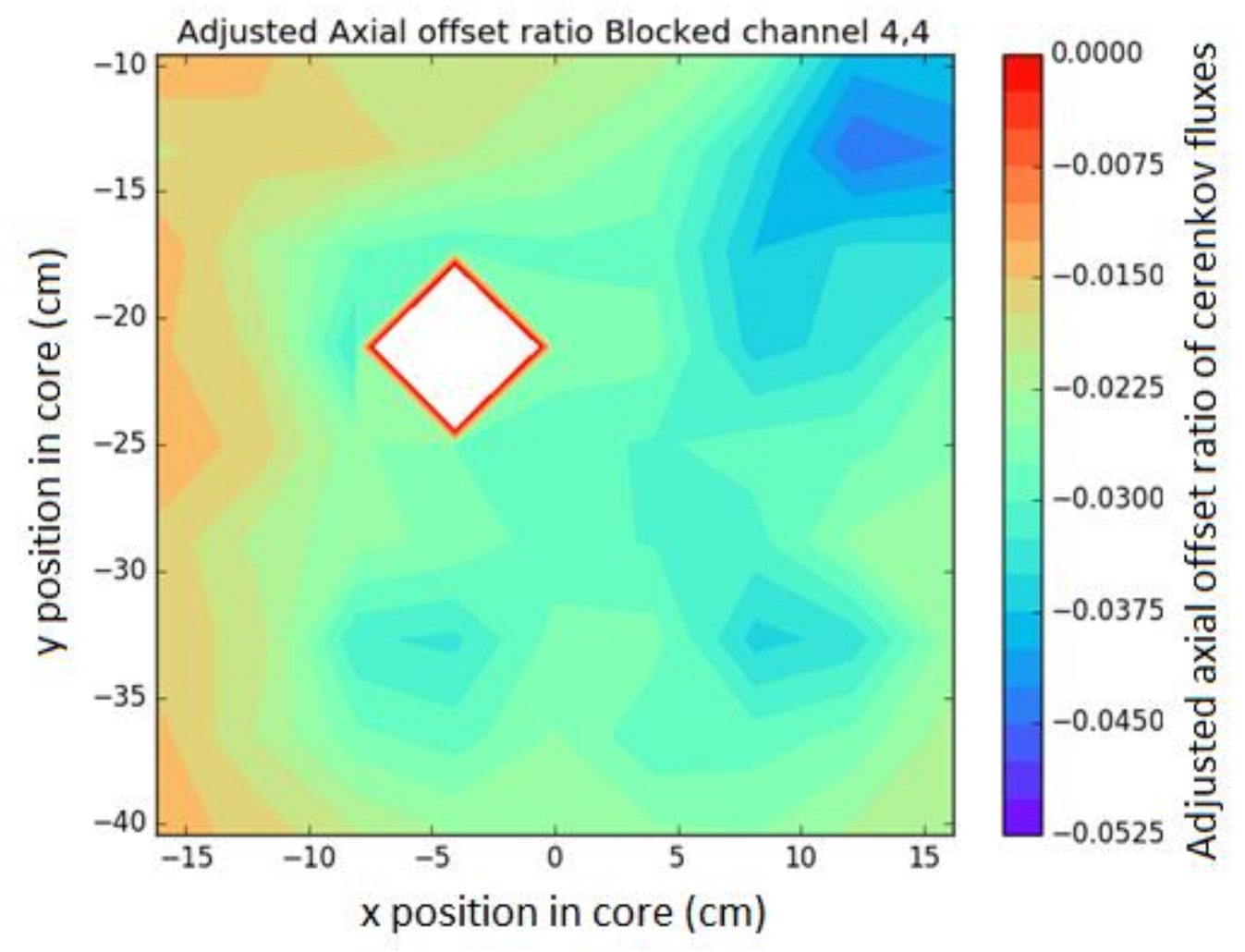

Figure 30 Axial tilt map of core with a $100 \%$ blockage at the core midplane in channel 4,4 . White space is where the tilt is outside of the previously used range. 


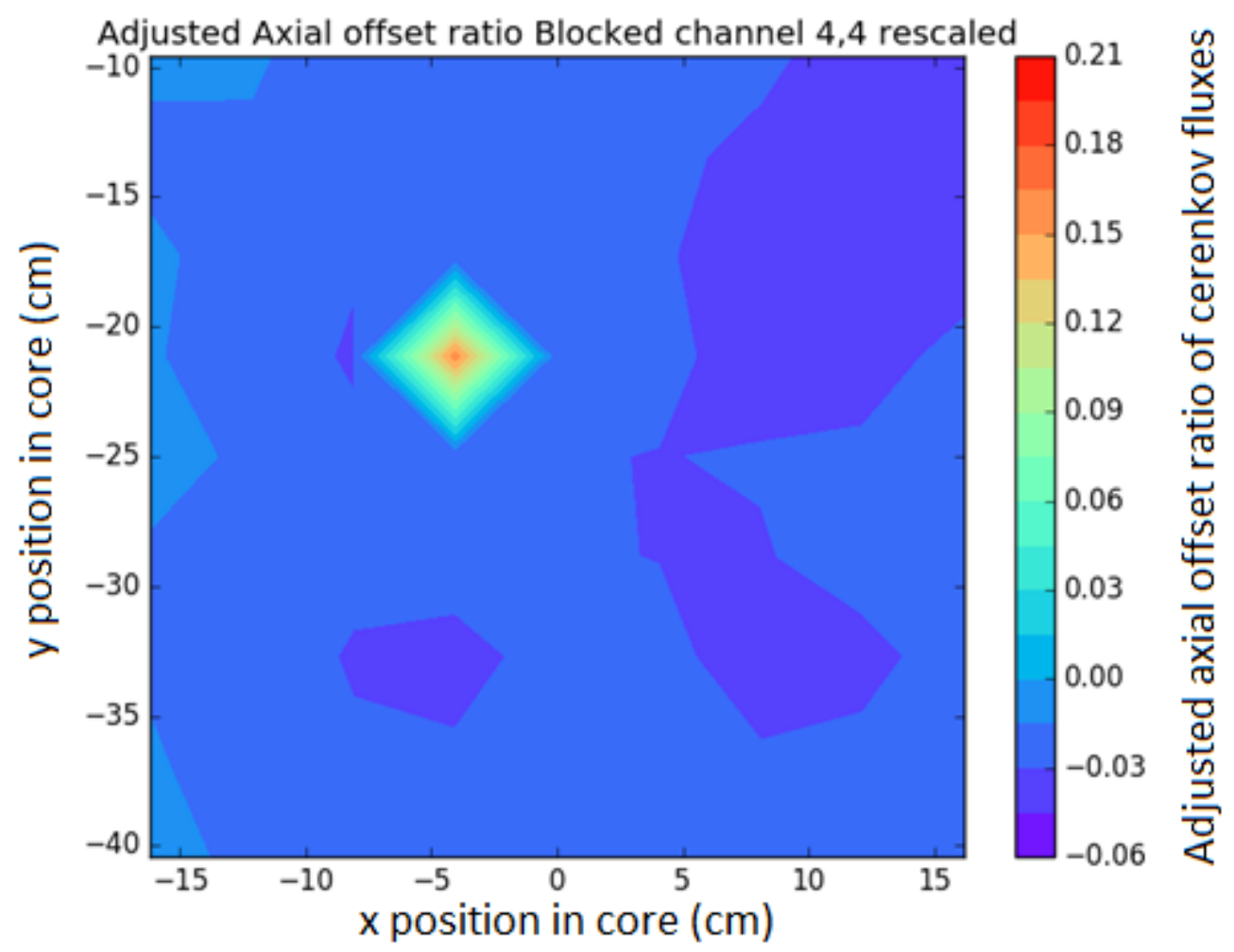

Figure 31 Rescaled plot of axial Cerenkov tilt with blocked channel. The large positive tilt in the affected channel stands out compared to the rest of the core.

To use the offset information shown above to identify the cause as a complete block near the midplane of the core, rather than a partial block near the top, it may be necessary to have more than one offset viewpoint. This is because a partial block could have a different effect on the offset viewpoint based upon its location within the channel. A partial block at the top of the channel on the side near the offset viewpoint would have a greater effect, while one opposite to the viewpoint would have a reduced effect; anything in between is also possible. If the assumption were made that a partial block would cause the same fractional reduction to the Cerenkov contributions below it for the direct viewpoint and the offset viewpoint, then a partial block near the top could be ruled out in 
by this information because it would cause no change in the tilt ratio. Multiple offset viewpoints in different directions with similar offset distances could be used to fix this. Instead of one offset viewpoint in the direction of a pin, four viewpoints, one in the direction of each pin neighboring the channel can be used, as seen in Figure 32. If the amount of light detected from a channel for each of four offset viewpoints were averaged, the assumption of an equal change to the direct viewpoint would be valid. Also, any major differences in the results detected by the different offsets can be used to determine which side of the channel a partial block is located. If the 4 offset viewpoints are affected asymmetrically by a partial block near the top of the channel, the block will be laterally positioned near the most affected offset viewpoint, because it will block the view of a larger portion of the channel. If the partial block is near the middle of the core, it would be laterally located opposite to the most affected offset, because in the nearest viewpoint, the blockage would be in the region already shadowed by the pin. This is demonstrated in two simple cases involving a 50\% block covering one side of the channel either at the top of the channel or at the midplane, seen in Figure 33, with the corresponding effects on the adjacent and opposite offset viewpoints. A block near the top cuts out $3 / 8^{\text {th }}$ of the total channel volume, or $3 / 4^{\text {th }}$ of the normally viewable channel volume for the nearby offset viewpoint, while only blocking $1 / 8^{\text {th }}$ of the total volume for the opposite viewpoint. The partial midplane block has no effect on the near viewpoint, but still blocks $1 / 8^{\text {th }}$ of the total channel volume for the opposite offset. For blockages that are not directly placed towards an offset viewpoint, a combination of all 4 would be used. An example of this is shown in the following section. 


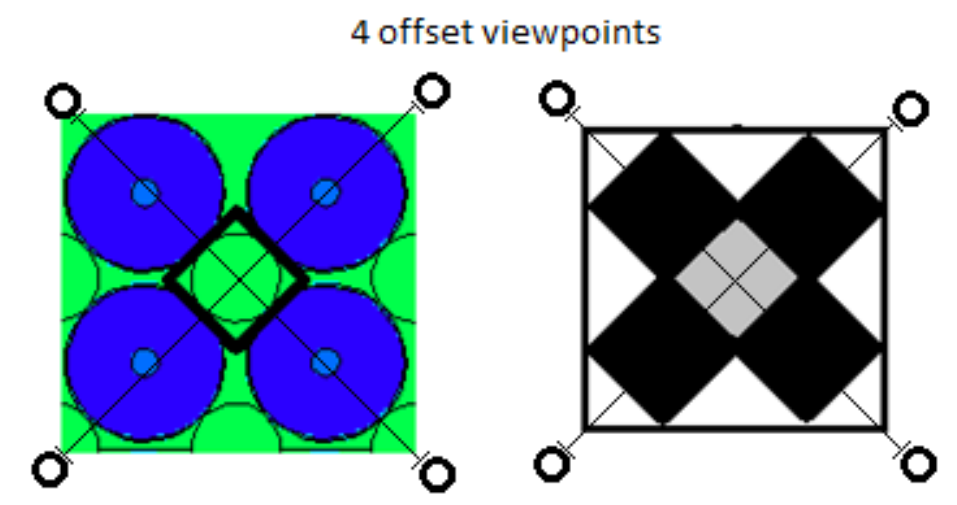

Figure 32 Pin geometry and square channel approximation using four offset viewpoints, one in the direction of each neighboring pin.

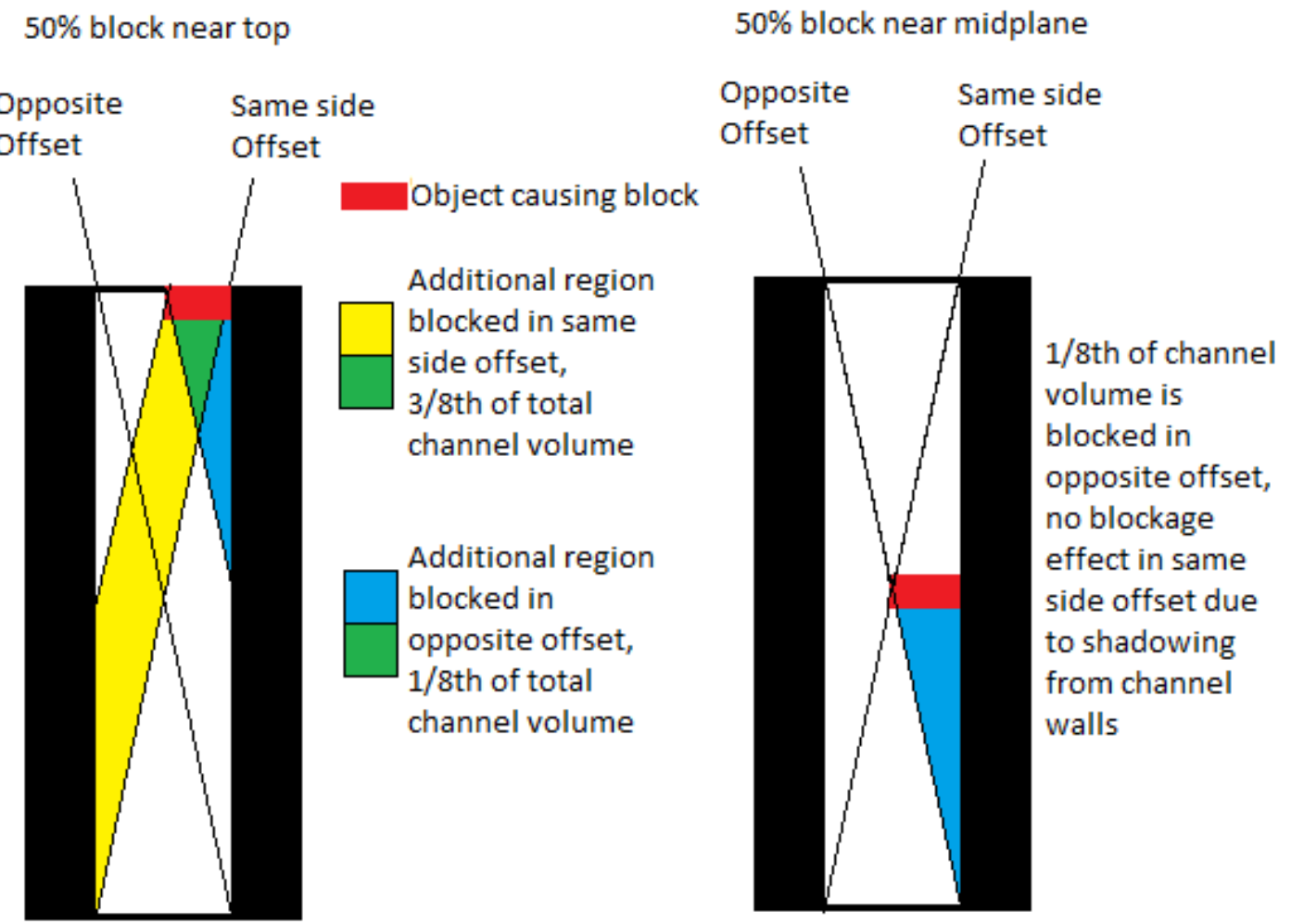

Figure 33 Diagram demonstrating the effect that a partial block in different locations within the channel has on the offset viewpoints located on the same side and on the opposite side of the channel from the blockage. 


\subsection{Example blockage detection and location}

An example case of the detection and location of a partial blockage within a channel using offset viewpoints is demonstrated here. For this example, there is an approximately $50 \%$ block at the core midplane on one side of channel 5,3 . The block is shown in Figure 34, along with the corresponding approximation used for the model of the Cerenkov detectors' response. The block is approximated as covering one side of the square coolant channel at the midplane.

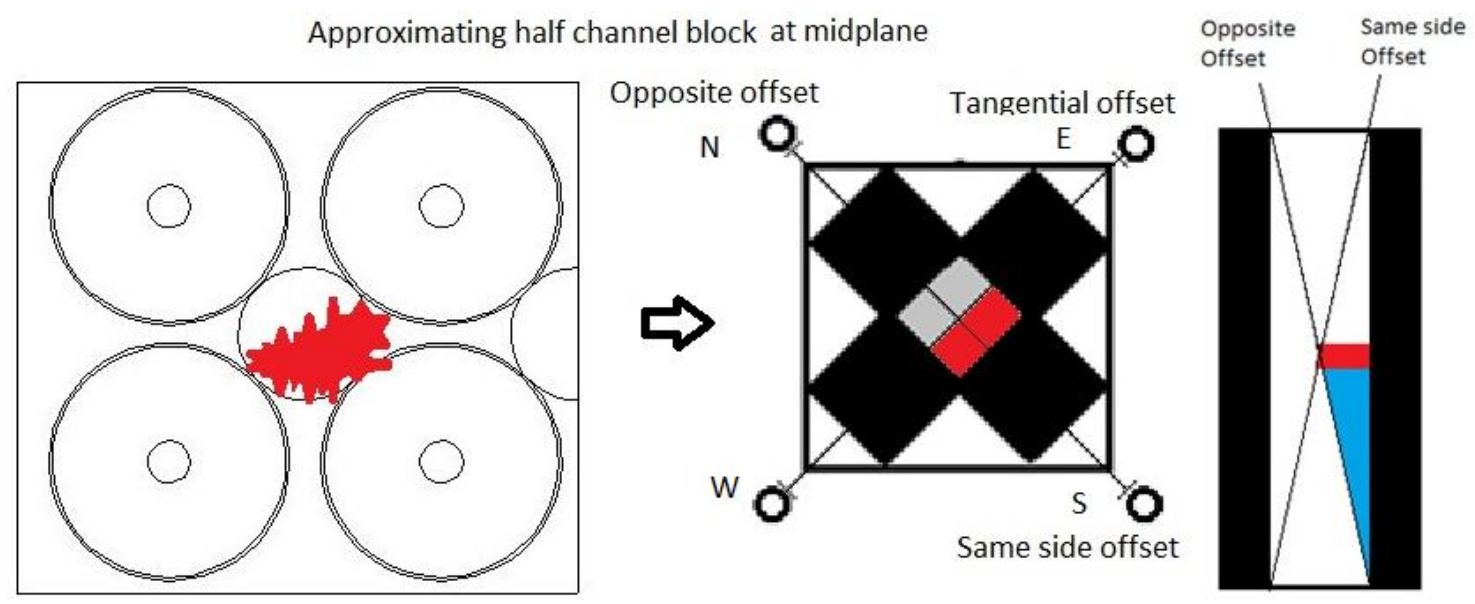

Figure 34 Partial blockage at core midplane and its approximation for the model

The normal level of Cerenkov without any blockage detected directly above channel 5,3 is $2.363 \mathrm{E}-7$, in units of Cerenkov photons per $\mathrm{cm}^{\wedge} 2$ per source neutron, as output by MCNP. This is altered by reducing the contribution of the lower half of the channel, segments 1 through 8 , by $50 \%$, due to the partial blockage at $\mathrm{z}=0 \mathrm{~cm}$. The resulting measurement directly above is $1.762 \mathrm{E}-7$. This is a decrease of $25.4 \%$, which is reasonable given that $1 / 4^{\text {th }}$ of the total channel volume is blocked and that the flux in most 
of the core is tilted downwards slightly, such that the slightly increased importance of the upper regions is countered by the higher prevalence of radiation in the lower regions.

The four offset viewpoints have different reactions to the blockage. For convenience here, they will be referred to North, South, East and West, with the block located on the southern side of the core, as labeled in Figure 34. With no block, all four offset viewpoints should have approximately the same Cerenkov measurements of 1.174E-7, corresponding to a tilt ratio of .4968 . Subtracting .5248 for normalization gives an adjusted tilt ratio of -.028 , corresponding to a slight downward tilt, as seen in previous tilt plots. With the blockage at the midplane, the offset viewpoint on the side where the block is present, the South side, actually cannot see the block, because it is in the shadow of the pin, so there is no reduction in the Cerenkov contributions of any segment and the value detected there is still 1.174E-7. This combined with the reduced direct viewpoint gives an adjusted tilt ratio of +0.141 for this offset, a very large apparent upwards tilt.

The two viewpoints tangential to the half channel block, the East and West views, will both have the contribution of segments 1 to 8 reduced by $50 \%$, because they see either their left or right side of the channel blocked. These should both have the same level of detected Cerenkov of $9.82 \mathrm{E}-8$, a decrease of only $16.3 \%$. The smaller decrease is because only $1 / 8^{\text {th }}$ of the total volume of view is blocked, because $3 / 4^{\text {th }}$ of the visible portion of the channel is above the midplane. This decrease is larger than $12.5 \%$ because the blocked portion contains mostly middle segments, which have more gammas and electrons, resulting in greater Cerenkov contributions. Comparing the blocked value for the tangential viewpoints to the blocked direct viewpoint gives an adjusted tilt ratio of 
+0.0325 , a much smaller apparent upwards tilt than for the viewpoint near the midplane block.

The viewpoint opposite to the $50 \%$ midplane block, the North view, has its Cerenkov contributions from segments 1 to 8 reduced to 0 , because the block covers the entire region that it can normally see below the midplane. This reduces the Cerenkov detection at this viewpoint to $7.91 \mathrm{E}-8$, a decrease of $32.6 \%$, because $1 / 4^{\text {th }}$ of the total visible volume is blocked, and that volume is mostly comprised of segments near the middle. This makes the apparent adjusted tilt ratio -0.0762 , which corresponds to a downwards tilt with a magnitude nearly $3 x$ larger than the normal, unblocked downwards tilt for that channel. The results for each offset are tabulated here in Table 1.

Table 1 Cerenkov detection in different viewpoints with $50 \%$ block in south side of channel at core midplane

\begin{tabular}{|c|c|c|c|c|c|c|}
\hline & $\begin{array}{l}\text { Direct } \\
\text { no } \\
\text { block }\end{array}$ & $\begin{array}{l}\text { Offset } \\
\text { no } \\
\text { block }\end{array}$ & $\begin{array}{l}\text { Direct } \\
\text { with } \\
\text { block }\end{array}$ & $\begin{array}{l}\text { Opposite } \\
\text { (north) } \\
\text { offset }\end{array}$ & $\begin{array}{l}\text { Same side } \\
\text { (south) } \\
\text { offset }\end{array}$ & $\begin{array}{l}\text { Tangential } \\
\text { (east/west) } \\
\text { offset }\end{array}$ \\
\hline $\begin{array}{l}\text { Cerenkov } \\
\text { detected }\end{array}$ & $2.36 \mathrm{E}-7$ & $1.17 \mathrm{E}-7$ & $1.76 \mathrm{E}-7$ & $7.91 \mathrm{E}-8$ & $1.17 \mathrm{E}-7$ & $9.82 \mathrm{E}-8$ \\
\hline Adj tilt ratio & N/a & -.0280 & N/a & -.0762 & +.1411 & +.0325 \\
\hline $\begin{array}{l}\text { Proportional } \\
\text { decrease in } \\
\text { Cerenkov }\end{array}$ & $\mathrm{N} / \mathrm{a}$ & $\mathrm{N} / \mathrm{a}$ & .254 & .3264 & 0 & .1632 \\
\hline
\end{tabular}


So combining the data from the direct and 4 offset detectors, the blockage causes Cerenkov detected from the direct view to be reduced by $25.4 \%$. The offsets are reduced by $0 \%$ to the South, $16.3 \%$ to the East and West, and $32.6 \%$ to the North: this information is what is available to find out where and how large the block is. The change in 4 of the 5 viewpoints can rule out a detector malfunction, so it is assumed that all simulated measurements are accurate. It is also assumed that there is only a single blockage, because two separate blockages is far less likely and more complicated. The blockage location process could proceed as follows:

The $25.4 \%$ decrease in the direct viewpoint means that the blockage is either a $25 \%$ block near the top below segment 16 , a $35 \%$ block below segment 11 , a $40 \%$ block below segment 10 , a $50 \%$ block at the midplane below segment 9 , a $100 \%$ block somewhere within segment 6 , at approximately $65 \%$ of the way down the channel, or some value in between.

The fact that there is no change in the Southern measurement means that the block must be completely within the region shadowed by the channel to the southern viewpoint, so it cannot be a block at the top, but instead must be a block smaller than or equal to the $\%$ of the way down the channel that the block is located. So to satisfy this criteria, $25 \%$ of the way down the channel, the block must cover $25 \%$ or less of the total channel area, at the midplane it must cover $50 \%$ or less of the channel, and a full block could only occur at the bottom of the channel. Taking the possible block sizes/positions from the direct viewpoint, the information from the South rules out the possibility of a $25 \%$ block at the top, or a $100 \%$ block at $\sim 65 \%$ of the way down the channel, but leaves the possibility of a 
$50 \%$ block at the midplane, located on the south side of the channel. Due to the slope of the curve for potential block sizes vs height from the direct viewpoint, the only place where it is within the region of shadow for the southern viewpoint is with a $50 \%$ block at the midplane, as anything above or below the region right around the midplane would have to be larger than the maximum size that could avoid blocking any of the southern view. Thus with only these two viewpoints, the size and location of the block in the channel can be identified.

The potential information obtainable from the other three offsets will also be mentioned here. The two tangential offsets, east and west, are both affected by the same amount, providing information that the block is symmetric with respect to the viewpoints: the line between the viewpoints is parallel to the edge of the blockage. The opposite, north, viewpoint has a larger decrease than all the others do, which shows that there is a block either on the north side near the top, or on the south side near the middle. This can provide further confirmation that it is indeed a block near the middle on the south side of the channel, because the other possibilities for creating this decrease in the north viewpoint are incompatible with the information from the other detectors.

Other sizes and positions of blocks could be determined from the Cerenkov measurements in a similar manner. A set of possible block configurations that would cause a given change in each viewpoint could be pre generated for many increments of change, and then an automated system could compare the possible configurations that would cause the response found in each viewpoint to find the blockage scenario that satisfies all of them, thus identifying the location of the block. 


\section{GENERALIZATION OF SYSTEM FOR USE IN OTHER REACTOR TYPES}

This section contains some discussion about how this method could be implemented in other reactors and what other reactor types could use it. The basic requirements for usage of the Cerenkov measuring power profile method are an optically transparent coolant and a straight line of sight through the coolant channel in the active fuel region and some distance above it.

\subsection{Reactor sensor calibration}

To ensure accuracy, every reactor sensor must be calibrated in some way. Reactor sensor calibration for power monitors is initially done using the calorimetric method [15]. In closed loop reactors, the calorimetric method obtains the reactor power be observing the change in temperature of the coolant between the inlet and outlet of the core. This change in temperature, combined with the mass flow rate of coolant through the core and the heat capacity of the coolant gives the rate at which heat is being added to the coolant and thus the total reactor power. In some pool type reactors, the calorimetric method instead uses the change in temperature of the pool over time, first using electric heaters of a known power to affect a change, and then using the reactor to create a change in the temperature of the pool [54]. The power obtained through the calorimetric method is then used to calibrate other power monitoring detectors by relating the output from the detectors to the known power level during the calibration.

Sensors must be periodically recalibrated to ensure that accuracy does not deteriorate over time. Historically, most sensor recalibration is done during refueling 
outages; efforts have been made to move towards online sensor calibration monitoring to reduce unnecessary recalibrations and shorten refueling and maintenance outages [55] [56] [57]. Digital systems can be used to perform fault detection and diagnosis by comparing sensor outputs to previous data, redundant sensors of the same type, or to analytical predictions based upon other sensor types [58] [59].

The calibration of a Cerenkov based power profile mapping system could be done in a similar way, thus relating the light detected to power measurements from other detectors. An additional system would provide more data to use in fault detection to further increase the reliability and to expand the types of faults detectable.

\subsection{Applicability to LWR}

A PWR is similar in some ways to the TRIGA reactor modeled here in that there are cylindrical fuel elements in a square lattice and the coolant is liquid water with no bulk boiling [15]. However, there are some differences that present additional challenges for the usage of a Cerenkov based power-profiling system for an operating PWR. The fuel pins are much smaller in a PWR and the spacing between them is correspondingly smaller. The fuel pins are also longer than in the TRIGA, so the length to spacing ratio is substantially larger. Additionally, the grid plates that hold the fuel pins in place can hinder visual access to the spaces between the pins. There is also limited space above the fuel assemblies due to the top of the pressure vessel and the presence of the various structures within the vessel above the active core region, limiting how far above the top of the fuel that a visual instrumentation viewpoint can be placed. These factors put much tighter 
constraints on the lateral location of viewpoints above coolant channels. The lack of vertical space above the fuel region could also cause the sensors to be in a stronger radiation field, due to the lack of shielding space between the fissions and the sensors. In general, the tight packing of a PWR and lack of additional space in the pressure vessel creates additional difficulty for the use of visual instrumentation, but it could still be done.

Another concern is that the coolant within a PWR undergoes nucleate boiling during operation. The hot channels can have a maximum void fraction close to $1 \%$ [60]. The presence of gas bubbles in water can significantly alter its reflectivity and transparency [61], which could cause significant changes in the amount of Cerenkov radiation reaching a sensor above the channel. If this were the case, the response in the sensor above the channel would fluctuate with the turbulent random nature of the fluid flow and would thus likely lose much of its value as a metric of power production. The effects of nucleate boiling on the transmissivity of the coolant would need to be investigated further before utilization of visual instrumentation in a PWR could be implemented.

A BWR would have a very significant problem with the boiling obscuring any line of sight through the core, and the moving bubbles causing a randomly fluctuating detector response. Therefore, a visual instrumentation system for online power monitoring in a BWR is not recommended.

Some small modular reactor designs [62] could be more easily fitted with visual instrumentation. The smaller core could more feasibly be covered by a single detector, and 
designs with additional space above the fuel assemblies could allow the detector to be outside of the stronger radiation fields.

\subsection{Applicability to FHR}

The Fluoride-Salt-Cooled High-Temperature Reactor (FHR) [63] [64] [65] is a reactor design that could make use of Cerenkov based visual instrumentation for power profile determination [66]. The primary salt considered as a coolant for the FHR is a Lithium-Beryllium-Fluoride (FLiBe) salt mixture with a 2 to 1 ratio of $\mathrm{LiF}$ to $\mathrm{BeF}_{2}$; however, some of the properties of FLiBe salt are still not fully characterized [67]. Specifically, the optical properties and index of refraction for FLiBe salt are either not known or not publically available. The optical properties of LiF salt are known; the index of refraction of solid $\mathrm{LiF}$ salt at room temperature for photons in the visible spectrum ranges from $\mathrm{n}=1.3996$ for $3.179 \mathrm{eV}$ photons to $\mathrm{n}=1.3902$ for $1.771 \mathrm{eV}$ photons [68]. The Molten Salts Handbook lists the refractive index of liquid LiF at $950 \mathrm{C}$ as 1.32 [69]. Refractive properties of $\mathrm{BeF}_{2}$ salt are not available. FliBe is $67 \% \mathrm{LiF}$ salt and the refractive index of LiF is similar to the refractive index of liquid water, 1.333, so it is assumed that the refractive properties of liquid FLiBe salt will be similar enough to produce usable Cerenkov responses. Accurate modeling of Cerenkov production in FLiBe salt would require data for the refractive properties, which could be obtained experimentally if a Cerenkov based power-profiling system for an FHR is pursued.

The geometry of an FHR is not yet fixed, due to the reactor being in the design stage. There are various different geometries considered for the FHR using tristructural 
isotropic (TRISO) particle based fuel. Some versions of the design use hexagonal fuel elements consisting of graphite fuel plates with rectangular coolant channels between the plates [70]. In these designs, the coolant channels run the entire axial length of the fueled region core. If the channels extend beyond the fueled region to a location where a detector can be placed, a straight line of sight through the core would be possible, allowing for the visual instrumentation method developed here to be implemented. Additionally, the rectangular coolant channels between the plates are much closer to the square channel approximations used in the offset viewpoint based 3D extrapolation, so it is expected that the use of offset viewpoints in these FHR assemblies could be implemented more accurately than in a TRIGA. One other potential advantage of visual instrumentation in an FHR is that the presence of any frozen FLiBe salt in the coolant channels would cause noticeable disturbances in the light detector responses, similar to a coolant channel blockage by other debris. The ability to detect another potential operational issue is an added benefit. Solidified salt is not expected to be present in the core or coolant loop under normal conditions, so it would not normally interfere with the instrumentation.

\subsection{Applicability to HTGR or other gas cooled designs}

High Temperature Gas-Cooled Reactors, (HTGRs) [71] are reactor designs using TRISO [72] particle fuel in a graphite matrix and helium gas as the coolant. Both prismatic fuel block [73] and pebble bed fueled designs exist. There are many similarities between the FHR and HTGR reactor designs, with the coolant choice as the primary difference. Pebble bed designs would not be as viable for Cerenkov based power instrumentation, 
because the arrangement of the fuel pebbles would preclude a straight line of sight through the core, and shifting of pebbles would change the amount of light reaching a detector. Prismatic fuel designs with straight coolant channels do have some potential for utilizing visual instrumentation.

One issue is that the index of refraction of helium at atmospheric pressure is 1.000035, lower than that of most other gasses. This causes an increase in the required electron energy for Cerenkov production, as the electrons must have a velocity greater than $\mathrm{c} / \mathrm{n}$, which in this case is $.999965 \mathrm{c}$, corresponding to an energy of $60 \mathrm{MeV}$. No electrons of this energy are expected to be present in the coolant of a reactor, so if the density of the helium gas was the same as it is at standard temperature and pressure, there would be no Cerenkov production. However, the difference between the index of refraction of an ideal gas and 1 is approximately proportional to the gas's density [74] [75], and the helium gas in HTGRs is generally at a high pressure. Of the various different HTGR designs used in the past, typical values for the coolant pressure and average temperature are around 4MPa and 600C [76]. In these conditions, the density of helium is $2.193 \mathrm{~kg} / \mathrm{m}^{3}$, more than 12 times the density at STP. The estimated index of refraction at this density is 1.00044 , which corresponds to a minimum electron velocity of $.99956 \mathrm{c}$ and an energy of $16.7 \mathrm{MeV}$ for Cerenkov production. This is still higher than the energy range of electrons produced by gamma interactions within the coolant of a reactor, so it is therefore expected that very little Cerenkov radiation would be produced in the coolant, preventing the use of this method for Cerenkov production based visual instrumentation in an HTGR. 


\subsection{Steps for implementation}

The process for implementing this method in power profile reconstruction procedures is summarized below.

On the physical side, a transparent coolant with a straight line of sight from the core to an external detection point is needed, likely through a coolant channel. Some form of photon detector that can survive the physical environment present at the detection point is needed. A transparent window of glass or some other material that can survive contact with the coolant at operating temperatures with chemically reacting would allow a detector to be somewhat insulated from the environment, though it will still likely be operating at a high temperature. The photon detectors can be installed at a number of locations, based upon the availability of locations and the desired resolution of the power profile map. A calibration of the responses from the photon detectors against other power measurement systems should also be done to accurately correlate the response to the power.

To implement the modelling portion of this method, a model of at least a coolant channel with a photon detector is needed to produce the correlation factors. The model can be run for a set of spatial and energy bins for the electron flux to relate the flux in the channel to a Cerenkov response above the channel, as described in section 2.3. This is the portion that requires the production and tracking of Cerenkov photons in the model, so simplicity is ideal to avoid any issues with MCNP's treatment of low energy photons.

A whole core model is needed to model the power profile and obtain the predicted corresponding Cerenkov responses. This model needs to track neutrons, gammas and electrons, but does not need to explicitly model Cerenkov photons. The coolant channels 
that have photon detectors monitoring them should have electron tallies with a bin structure that matches up to the correlation factors. Separate, multi-bin tallies for each channel being observed are needed. The results of these electron tallies can then be combined with the correlation factors from the simple model to estimate the Cerenkov flux detected by the photon detectors. Calibrating this against data from the real, physical detection system will likely be necessary for accurate application.

Any form of 3D extrapolation using multiple viewpoints is dependent upon the reactor design. Geometric considerations for how each segment of a coolant channel contributes to the offset viewpoint's Cerenkov detection will need to be accounted for. An adjustment to the contribution of each segment to the total Cerenkov detected at an offset would allow the model to predict the offset viewpoint photon fluxes as well.

Once a model has been generated and the calibration performed, the model could be used to analyze many different operational or accident scenarios to generate a library of expected responses in the Cerenkov detection system, such that when a similar scenario occurs in the reactor during operation, it will be possible to identify what it is based upon the similarity in the response of the detectors to the expected response from previously run models. 


\section{CONCLUSIONS}

A method for determining the power profile with visual instrumentation in a reactor with an optically transparent coolant has been developed. The TRIGA reactor at Texas A\&M is used as a surrogate case for any reactor with a transparent coolant. This method uses visible light detectors above the core to measure the Cerenkov radiation coming from coolant channels to provide information about the power density in the pins surrounding the channels. This can create a 2D map of the power density in the core, which has been demonstrated to detect changes in the power profile caused by changes in rod positions, coolant channel blockages, and changes in the position of the reactor within the pool.

Information about the Cerenkov radiation detected at offset viewpoints that are not directly above the coolant channels can be combined with the direct viewpoint data to determine the axial tilt of the power within the core around each channel. This allows a plot of the flux tilt in each channel to be produced, giving an approximate 3D power profile within the core. A blockage of a coolant channel can be easily detected, and using the offset viewpoints, the location and size of the block within the channel can also be approximately determined.

Further work to be done on developing this method is primarily in the direction of reactor specific application and designing the physical detection system. This research effort has been entirely model and theory based and has not yet been experimentally validated. To apply this to another reactor, a set of models of the reactor would need to be produced in a manner similar to what is done here. A physical system of photon detectors 
that can survive the reactor environment is necessary for this to be implemented; the experimental aspects of development have not been pursued in this dissertation. Additionally, the creation of a wider library of reactor states and their corresponding Cerenkov responses could be produced and some form of recognition software developed to automatically identify the reactor state based upon an observed Cerenkov response. 


\section{REFERENCES}

[1] G. Knoll, Radiation Detection and Measurement (4th ed.), Wiley, 2010.

[2] J. J. Loving, "Neutron, Temperature and Gamma Sensors for Pressurized Water Reactors," IEEE Transactions on Industrial Electronics and Control Instrumentation, vol. 17, no. 2, pp. 120-129, 1970.

[3] J. J. Forster, "In-Core Neutron Flux Instrumentation," IEEE Transactions on Nuclear Science, vol. 12, no. 6, pp. 8-14, 1965.

[4] D. E. Holcomb, R. A. Kisner and S. M. Cetiner, "Instrumentation Framework for Molten Salt Reactors," Oak Ridge National Laboratory, June 2018.

[5] H. M. Hashemian and J. Jiang, "Nuclear plant temperature instrumentation," Nuclear Engineering and Design, vol. 239, no. 12, pp. 3132-3141, 2009.

[6] N. Sion, "In-core moderator temperature measurement within candu reactors," Nuclear Instruments and Methods in Physics Research, vol. 206, no. 3, pp. 527$536,1983$.

[7] C. D. Henning and R. Parker, "Transient Response of an Intrinsic Thermocouple," Journal of Heat Transfer, vol. 89, no. 2, pp. 146-152, 1966.

[8] J. F. Villard, S. Fourrez, D. Fourmentel and A. Legrand, "Transient Response of an Intrinsic Thermocouple," International Journal of Thermophysics, vol. 29, no. 5, pp. 1848-1857, 2008. 
[9] A. Kimura, E. Takada, K. Fujita, M. Nakazawa, H. Takahashi and S. Ichige, "Application of a Raman distributed temperature sensor to the experimental fast reactor JOYO with correction techniques," Measurement Science and Technology, vol. 12 , no. 7,2001 .

[10] R. A. Ali, S. L. Garret, J. A. Smith and D. K. Kotter, "Thermoacoustic thermometry for nuclear reactor monitoring," IEEE Instrumentation \& Measurement Magazine, vol. 16, no. 3, pp. 18-25, 2013.

[11] S. L. Garrett, J. A. Smith, R. W. M. Smith, B. J. Heidrich and M. D. Heibel, "Fission-powered in-core thermoacoustic sensor," Applied Physics Letters, vol. 108, no. 14, 2016.

[12] K. Tsai, C. Jensen, T. Unruh, A. Fleming and C. Race, "In-Core Evaluation of Online Insrumentation in the TREAT Reactor," in Transactions of the American Nuclear Society, Vol. 118, Philadelphia, Pennsylvania, 2018.

[13] E. Udd and W. B. Spillman, Fiber Optic Sensors, An Introduction for Engineers and Scientists Second Edition, Hohoken, New Jersey: John Wiley \& Sons, 2011.

[14] G. Grosshoeg, "Neutron Ionization Chambers," Nuclear Instruments and Methods, vol. 162, pp. 125-160, 1979.

[15] Westinghouse, Westinghouse Technology Systems Manual, USNRC HRTD.

[16] D. S. McGregor, M. L. Adams, I. Carron and P. Nelson, "Near-Core and In-Core Neutron Radiation Monitors for Real Time Neutron Flux Monitoring and Reactor Power Level Measurements," Kansas State University, 2006. 
[17] W. M. Trenholme and D. J. Keefe, "A Neutron Flux Measuring Channel Covering Ten Decades of Reactor Power with a Single Fixed-Position Detector," IEEE Transactions on Nuclear Science, vol. 14, no. 1, pp. 253-260, 1967.

[18] K. R. Prasad, P. M. Dighe and M. Alex, "Boron-lined neutron detectors with gamma discrimination for reactor applications," IJEMS, vol. 7, no. 1, pp. 21-24, 2000.

[19] C. N. Jackson, "Reactor In-Core regenerative neutron detectors interim development report," Pacific Northwest Laboratory, Richland, Washington, 1967.

[20] C. Jammes, P. Filliatre, B. Geslot, L. Oriol, F. Berhouet, J.-F. Villard and L. Vermeeren, "Research Activities in Fission Chamber Modeling in Support of the Nuclear Energy Industry," IEEE Transactions on Nuclear Science, vol. 57, no. 6, pp. 3678-3682, 2010.

[21] J. W. Hilborn, "Self-powered neutron netector". United States Patent US3375370A, 1965.

[22] N. P. Goldstein and W. H. Todt, "A Survey of Self-Powered Detectors - Present and Future," IEEE Transactions on Nuclear Science, Vols. NS-26, no. 1, pp. 916923, 1979.

[23] W. H. Todt, "Characteristics of self-powered neitron detectors used in power reactors," in Proc. of the Specialists' Meeting on Incore Instrumentation and Reactor Core Assessment, Mito City, Japan, 1996. 
[24] A. K. Mishra, S. R. Shimjith, T. U. Bhatt and A. P. Tiwari, "Dynamic Compensation of Vanadium Self Powered Neutron Detectors for Use in Reactor Control," IEE transactions on Nuclear Science, vol. 60, no. 1, pp. 310-318, 2013.

[25] M. A. Reichenberger, T. C. Unruh, P. B. Ugorowski, T. Ito, J. A. Roberts, S. R. Stevenson, D. M. Nichols and D. S. McGregor, "Micro-Pocket Fission Detectors (MPFDs) for in-core neutron detection," Annals of Nuclear Energy, vol. 87 Part 2, pp. 318-323, January 2016.

[26] T. Unruh, M. Reichenberger, S. Stevenson, D. McGregor, K. Tsal and J. F. Villard, "Enhanced Micro-Pocket Fission Detector for High Temperature Reactors," Advanced Sensors and Instrumentation, no. 7, pp. 1-3, 2017.

[27] V. K. Patel, M. A. Reichenberger, J. A. Roberts, T. C. Unruh and D. S. McGregor, "MCNP6 simulated performance of Micro-Pocket Fission Detectors (MPFDs) in the Transient REActor Test (TREAT) facility," Annals of Nuclear Energy, vol. 104, pp. 191-196, June 2017.

[28] D. S. McGregor, M. F. Ohmes, R. E. Ortiz, A. S. Ahmed and J. K. Shultis, "Micropocket fission detectors (MPFD) for in-core neutron flux monitoring," Nuclear Instruments and Methods in Physics Research Section A: Accelerators, Spectrometers, Detectors and Associated Equipment, vol. 554, no. 1-3, pp. 494499, 2005. 
[29] F. H. Ruddy, A. R. Dullo, J. G. Seidel, F. W. Hantz and L. R. Grobmyer, "Nuclear Reactor Power Monitoring Using Silicon Carbide Semiconductor Radiation Detectors," Nuclear Technology, vol. 140, no. 2, pp. 198-208, 2002.

[30] M. jalali, M. R. Abdil and M. M. Davati, "Prompt gamma radiation as a new tool to measure reactor power," Radiation Physics and Chemistry, vol. 91, pp. 19-27, 2013.

[31] K. G. Porges, R. Gold and W. C. Corwin, "Reactor Power Monitor Based on Cherenkov Radiation Detection," IEEE Transactions on Nuclear Science, vol. 17, no. 1 , pp. 501-505, 1970 .

[32] J. R. Humphries and J. M. Carpenter, "Gaseous Cerenkov Detector Reactor Power Monitor," IEEE Transactions on Nuclear Science, vol. 19, no. 1, pp. 807-813, 1972.

[33] Y. V. Klimov, V. I. Kopeikin, L. A. Mikaélyan and V. V. Sinev, "Neutrino method remote measurement of reactor power and power output," Atomic Energy, vol. 76, no. 2, pp. $123-127,1994$.

[34] S. E. Rippon, "Cherenkov detectors for the measurement of reactor power," Nucl. Ins. Methods, vol. 21, pp. 192-196, 1963.

[35] M. Arakani and M. Gharib, Design and Construction of an Independent Channel for Tehran Research Reactor Power Measurement Using Cherenkov Detector, M. S. Thesis, Azad University, Research and Science Department, Tehran, 2007. 
[36] M. Arakani and M. Gharib, "Reactor core power measurement using Cherenkov radiation and its application in Tehran Research Reactor," Ann. Nucl. Eng., vol. 36, pp. 869-900, 2009.

[37] J. L. Lovvorn, "Reactor Power Monitor Utilizing Cerenkov Radiation," IRE Transactions on Nuclear Science, vol. 8, no. 4, pp. 3-5, Oct. 1961.

[38] G. Bentoumi, B. Benson, P. K. Chan, M. Gaudet, L. Li, P. Samuleev and B. Sur, "Reactor power monitoring using Cherenkov radiation transmitted through a smallbore metallic tube," Annals of Nuclear Energy, vol. 114, pp. 86-91, April 2018.

[39] H. Weiss, "Power measurement and automatic reactor control by gamme - or cerenkov - radiation," in Nuclear Electronics, Vienna, Austria, 1965.

[40] Safeguards Techniques and Equipment 2011 Edition, Vienna: IAEA.

[41] S. Farley, "An overview of non destructive inspection services in nuclear power plants," in Proceedings of the International Conference Nuclear Energy for New Europe 2004, 2004.

[42] BWR Refuling Machine Camera N180-TZ. [Film]. Ahlberg Cameras, 2012.

[43] A. Horvath, Artist, The geometry of the Cherenkov radiation (shown for the ideal case of no dispersion). [Art]. 2006.

[44] T. I. Quickenden and J. A. Irving, "The ultraviolet absorption spectrum of liquid water," The journal of Chemical Physics, 72, 4416, 1980. 
[45] R. A. J. Litjens, T. I. Quickenden and C. G. Freeman, "Visible and near-ultraviolet absorption spectrum of liquid water," Applied Optics, vol. 38, no. 7, pp. 1216$1223,1999$.

[46] L. V. Wang and H. I. Wu, Biomedical Optics, Wiley, 2007.

[47] T. Goorley, "MCNP6.1.1-Beta Release Notes," Los Alamos National Lab, 2014.

[48] J. W. Durkee and M. R. James, "MCNP6 Cerenkov Radiation Feature Verification," Los Alamos National Lab, Los Alamos, New Mexico, 2013-07-17.

[49] Y. Shimazu, "Simplest Simulation Model for Three-Dimensional Xenon Oscillations in Large PWRs," Journal of nuclear science and technology, vol. 41, no. 10, pp. 959-965, 2004.

[50] H. Parhizkari, M. Aghaie, A. Zolfaghari and A. Minuchehr, "An approach to stability analysis of spatial xenon oscillations in WWER-1000 reactors," Annals of Nuclear Energy, vol. 79, pp. 125-132, May 2015.

[51] A. A. El-Bassioni and C. G. Poncelet, "Minimal Time Control of Spatial Xenon Oscillations in Nuclear Power Reactors," Nuclear Science and Engineering, vol. 54, no. 2, pp. 166-176, 1974.

[52] J. Chernick, G. Lellouche and W. Wollman, "The Effect of Temperature on Xenon Instability," Nuclear Science and Engineering, vol. 10, no. 2, pp. 120-131, 1961.

[53] S. R. Shimjith, A. P. Tiwari, M. Naskar and B. Bandyopadhyay, "Space-time kinetics modeling of Advanced Heavy Water Reactor for control studies," Annals of Nuclear Energy, vol. 37, no. 3, pp. 310-324, 2010. 
[54] A. Z. Mesquita, H. C. Rezende and E. B. Tambourgi, "Power calibration of the TRIGA mark I nuclear research reactor," Journal of the Brazilian Society of Mechanical Sciences and Engineering, vol. 29, no. 3, pp. 240-245, 2007.

[55] J. B. Coble, R. M. Meyer, P. Ramuhalli, L. J. Bond, H. Hashemian, B. Shumaker and D. Cummins, "A Review of Sensor Calibration Monitoring for Calibration Interval Extension in Nuclear Power Plants," Pacific Northwest National Lab, Richland, WA, 2012.

[56] H. Hashemian, "On-line testing of calibration of process instrumentation channels in nuclear power plants. Phase 2, Final report," NRC, 1995.

[57] J. Hines and E. Davis, "Lessons learned from the U.S. nuclear power Plant on-line monitoring programs," Progress in Nuclear Energy, vol. 46, no. 3-4, pp. 176-189, 2005.

[58] J. Ma and J. Jiang, "Applications of fault detection and diagnosis methods in nuclear power plants: A review," Progress in Nuclear Energy, vol. 53, no. 3, pp. 255-266, 2011.

[59] J. A. Bernard, D. D. Lanning and A. Ray, "Digital Control of Power Transients in a Nuclear Reactor," IEEE Transactions on Nuclear Science, vol. 31, no. 1, pp. 701$705,1984$.

[60] Westinghouse, AP1000 Design Control Document, NRC.

[61] S. Russell, "Bright Water: Hydrosols, Water Conservation and Climate Change," Harvard, Cambridge, MA, 2010. 
[62] Z. Liu and J. Fan, "Technology readiness assessment of Small Modular Reactor (SMR) designs," Progress in Nuclear Energy, vol. 70, pp. 20-28, 2014.

[63] C. Forsberg, L.-W. Hu, P. Peterson and K. Sridharan, "Fluoride-Salt-Cooled HighTemperature Reactor (FHR) for Power and Process Heat," Nuclear Energy University Programs, 2014.

[64] R. O. Scarlat and E. al., "Design and licensing strategies for the fluoride-saltcooled, high-temperature reactor (FHR) technology," Progress In Nuclear Energy, vol. 77, pp. 406-420, November 2014.

[65] N. R. Brown, B. R. Betzler, J. J. Carbajo, A. J. Wysocki, M. S. Greenwood, C. Gentry and A. L. Qualls, "Preconceptual design of a fluoride high temperature saltcooled engineering demonstration reactor: Core design and safety analysis," Annals of Nuclear Energy, vol. 103, pp. 49-59, 2017.

[66] D. E. Holcomb, S. M. Cetiner, G. F. Flanagan, F. J. Peretz and G. L. J. Yoder, "AN ANALYSIS OF TESTING REQUIREMENTS FOR FLUORIDE SALT-COOLED HIGH TEMPERATURE REACTOR COMPONENTS," Oak Ridge National Laboratory, Oak Ridge, Tennessee, 2009.

[67] R. R. Romatoski and L. W. Hu, "Fluoride salt coolant properties for nuclear reactor applications: A review," Annals of Nuclear Energy, vol. 109, pp. 635-647, 2017.

[68] E. D. Palik and W. R. Hunter, "Lithium Fluoride (LiF)," in Handbook of Optical Constants of Solids, Washington, D.C, Naval Research Laboratory , 1997, pp. 675693. 
[69] G. J. Janz, "I.F - REFRACTIVE INDEX," in Molten Salts Handbook, Academic Press, 1967, pp. 89-92.

[70] K. M. Ramey and B. Petrovic, "Monte Carlo modeling and simulations of AHTR fuel assembly to support V\&V of FHR core physics methods," Annals of Nuclear Energy, vol. 118, pp. 272-282, 2018.

[71] R. A. Moore, M. E. Kantor, H. L. Brey and H. G. Olson, "HTGR experience, programs, and future applications," Nuclear Engineering and Design, vol. 72, no. 2, pp. 153-174, 1982.

[72] P. A. Demkowicz, B. Liu and J. D. Hunn, "Coated particle fuel: Historical perspectives and current progress," Journal of Nuclear Materials, 2018.

[73] P. H. Liem and H. Sekimoto, "Small high temperature gas-cooled reactors with innovative nuclear burning," Progress in Nuclear Energy, vol. 50, no. 2-6, pp. 251256, 2008.

[74] B. H. Sang and T.-I. Jeon, "Pressure-dependent refractive indices of," Optics Express, vol. 24, no. 25, 2016.

[75] A. Michels and J. Hamers, "The effect of pressure on the refractive index of CO2: The Lorentz-Lorenz formula," Physica, vol. 4, no. 10, pp. 995-1006, 1937.

[76] B. Mcdowell, M. Mitchell, R. Pugh, J. Nockolaus and G. Swearinged, "High Temperature Gas Reactors:," Pacific Northwest National Lab, 2011. 


\section{APPENDIX A}

\section{ELECTRON TO CERENKOV FLUX CORRELATION FACTORS}

This table shows the correlation factors for the amount of visible Cerenkov radiation observed $2 \mathrm{~m}$ above the core midplane produced by electrons in a segment with energies within a specified range. Only electrons within the segment and energy range contribute to the Cerenkov total for each factor. A separate MCNP deck was run for each energy bin and spatial bin, such that electrons are spawned in the region with an energy distribution in the energy bin and electrons that leave the region or drop below the energy cutoff of the bin are killed. The method of obtaining the factors is also described in section

\section{3, Electron to Cerenkov Flux Correlations.}

The segment number and the $\mathrm{z}$ position of the upper bound and lower bound of each segment are shown in the first 3 columns. The minimum and maximum energy for each electron energy bin are shown in columns 4 and 5. Column 6, pflux/eflux shows the ratio of the photon flux $2 \mathrm{~m}$ above the core caused by electrons in the segment and energy bin to the flux of electrons in that bin. Column 7 has the relative uncertainty, which is the uncertainty obtained by propagating the uncertainties output from MCNP for the photon flux and electron flux. The bottommost (segment 1) and topmost (segment 16) segments were not initially run in the simulations, and are instead duplicates of the segments 2 and 15. This adds a very little error because the difference between neighboring segments is small, and the contribution of the end segments to the total is a small portion of the whole, due to the lower gamma and electron fluxes further from the core midplane. 
Table 2 Electron to Cerenkov flux correlations within a coolant channel

\begin{tabular}{|c|c|c|c|c|c|c|}
\hline $\begin{array}{l}\text { Segment } \\
\text { number }\end{array}$ & $\begin{array}{l}\mathrm{z} \\
\text { bottom }\end{array}$ & $z$ top & $\begin{array}{l}\text { Min } \\
\text { Energy } \\
(\mathrm{keV})\end{array}$ & $\begin{array}{l}\text { Max } \\
\text { Energy } \\
(\mathrm{keV})\end{array}$ & pflux/ eflux & $\begin{array}{l}\text { Relative } \\
\text { uncertainty }\end{array}$ \\
\hline 1 & $-29 \mathrm{~cm}$ & $-28 \mathrm{~cm}$ & 200 & 300 & 0.000156383 & 0.267300019 \\
\hline 1 & $-29 \mathrm{~cm}$ & $-28 \mathrm{~cm}$ & 300 & 400 & 0.001319191 & 0.085400059 \\
\hline 1 & $-29 \mathrm{~cm}$ & $-28 \mathrm{~cm}$ & 400 & 500 & 0.002526843 & 0.059100085 \\
\hline 1 & $-29 \mathrm{~cm}$ & $-28 \mathrm{~cm}$ & 500 & 600 & 0.002855936 & 0.054200092 \\
\hline 1 & $-29 \mathrm{~cm}$ & $-28 \mathrm{~cm}$ & 600 & 700 & 0.003186101 & 0.0502001 \\
\hline 1 & $-29 \mathrm{~cm}$ & $-28 \mathrm{~cm}$ & 700 & 800 & 0.003427128 & 0.047800105 \\
\hline 1 & $-29 \mathrm{~cm}$ & $-28 \mathrm{~cm}$ & 800 & 900 & 0.0035331 & 0.046600107 \\
\hline 1 & $-29 \mathrm{~cm}$ & $-28 \mathrm{~cm}$ & 900 & 1000 & 0.003657704 & 0.04550011 \\
\hline 1 & $-29 \mathrm{~cm}$ & $-28 \mathrm{~cm}$ & 1000 & 1100 & 0.003826298 & 0.043900114 \\
\hline 1 & $-29 \mathrm{~cm}$ & $-28 \mathrm{~cm}$ & 1100 & 1200 & 0.003938364 & 0.042500118 \\
\hline 1 & $-29 \mathrm{~cm}$ & $-28 \mathrm{~cm}$ & 1200 & 1300 & 0.004248154 & 0.040500123 \\
\hline 1 & $-29 \mathrm{~cm}$ & $-28 \mathrm{~cm}$ & 1300 & 1400 & 0.004095292 & 0.041100122 \\
\hline 1 & $-29 \mathrm{~cm}$ & $-28 \mathrm{~cm}$ & 1400 & 1500 & 0.004109203 & 0.040600123 \\
\hline 1 & $-29 \mathrm{~cm}$ & $-28 \mathrm{~cm}$ & 1500 & 1600 & 0.004222942 & 0.039900125 \\
\hline 1 & $-29 \mathrm{~cm}$ & $-28 \mathrm{~cm}$ & 1600 & 1700 & 0.004312454 & 0.038800129 \\
\hline 1 & $-29 \mathrm{~cm}$ & $-28 \mathrm{~cm}$ & 1700 & 1800 & 0.004209801 & 0.039000128 \\
\hline
\end{tabular}




\begin{tabular}{|c|c|c|c|c|c|c|}
\hline $\begin{array}{l}\text { Segment } \\
\text { number }\end{array}$ & $\begin{array}{l}\text { z } \\
\text { bottom }\end{array}$ & z top & $\begin{array}{l}\text { Min } \\
\text { Energy } \\
(\mathrm{keV})\end{array}$ & $\begin{array}{l}\text { Max } \\
\text { Energy } \\
\text { (keV) }\end{array}$ & pflux/eflux & $\begin{array}{l}\text { Relative } \\
\text { uncertainty }\end{array}$ \\
\hline 1 & $-29 \mathrm{~cm}$ & $-28 \mathrm{~cm}$ & 1800 & 1900 & 0.004007741 & 0.040000125 \\
\hline 1 & $-29 \mathrm{~cm}$ & $-28 \mathrm{~cm}$ & 1900 & 2000 & 0.004253328 & 0.03840013 \\
\hline 1 & $-29 \mathrm{~cm}$ & $-28 \mathrm{~cm}$ & 2000 & 2100 & 0.00440218 & 0.034001324 \\
\hline 1 & $-29 \mathrm{~cm}$ & $-28 \mathrm{~cm}$ & 3000 & 4000 & 0.004377412 & 0.033901327 \\
\hline 1 & $-29 \mathrm{~cm}$ & $-28 \mathrm{~cm}$ & 4000 & 5000 & 0.00443812 & 0.033801331 \\
\hline 1 & $-29 \mathrm{~cm}$ & $-28 \mathrm{~cm}$ & 5000 & 10000 & 0.004463775 & 0.032207608 \\
\hline 2 & $-28 \mathrm{~cm}$ & $-24 \mathrm{~cm}$ & 200 & 300 & 0.000156383 & 0.267300019 \\
\hline 2 & $-28 \mathrm{~cm}$ & $-24 \mathrm{~cm}$ & 300 & 400 & 0.001319191 & 0.085400059 \\
\hline 2 & $-28 \mathrm{~cm}$ & $-24 \mathrm{~cm}$ & 400 & 500 & 0.002526843 & 0.059100085 \\
\hline 2 & $-28 \mathrm{~cm}$ & $-24 \mathrm{~cm}$ & 500 & 600 & 0.002855936 & 0.054200092 \\
\hline 2 & $-28 \mathrm{~cm}$ & $-24 \mathrm{~cm}$ & 600 & 700 & 0.003186101 & 0.0502001 \\
\hline 2 & $-28 \mathrm{~cm}$ & $-24 \mathrm{~cm}$ & 700 & 800 & 0.003427128 & 0.047800105 \\
\hline 2 & $-28 \mathrm{~cm}$ & $-24 \mathrm{~cm}$ & 800 & 900 & 0.0035331 & 0.046600107 \\
\hline 2 & $-28 \mathrm{~cm}$ & $-24 \mathrm{~cm}$ & 900 & 1000 & 0.003657704 & 0.04550011 \\
\hline 2 & $-28 \mathrm{~cm}$ & $-24 \mathrm{~cm}$ & 1000 & 1100 & 0.003826298 & 0.043900114 \\
\hline 2 & $-28 \mathrm{~cm}$ & $-24 \mathrm{~cm}$ & 1100 & 1200 & 0.003938364 & 0.042500118 \\
\hline 2 & $-28 \mathrm{~cm}$ & $-24 \mathrm{~cm}$ & 1200 & 1300 & 0.004248154 & 0.040500123 \\
\hline
\end{tabular}




\begin{tabular}{|c|c|c|c|c|c|c|}
\hline $\begin{array}{l}\text { Segment } \\
\text { number }\end{array}$ & $\begin{array}{l}\text { z } \\
\text { bottom }\end{array}$ & z top & $\begin{array}{l}\text { Min } \\
\text { Energy } \\
(\mathrm{keV})\end{array}$ & $\begin{array}{l}\text { Max } \\
\text { Energy } \\
\text { (keV) }\end{array}$ & pflux/eflux & $\begin{array}{l}\text { Relative } \\
\text { uncertainty }\end{array}$ \\
\hline 2 & $-28 \mathrm{~cm}$ & $-24 \mathrm{~cm}$ & 1300 & 1400 & 0.004095292 & 0.041100122 \\
\hline 2 & $-28 \mathrm{~cm}$ & $-24 \mathrm{~cm}$ & 1400 & 1500 & 0.004109203 & 0.040600123 \\
\hline 2 & $-28 \mathrm{~cm}$ & $-24 \mathrm{~cm}$ & 1500 & 1600 & 0.004222942 & 0.039900125 \\
\hline 2 & $-28 \mathrm{~cm}$ & $-24 \mathrm{~cm}$ & 1600 & 1700 & 0.004312454 & 0.038800129 \\
\hline 2 & $-28 \mathrm{~cm}$ & $-24 \mathrm{~cm}$ & 1700 & 1800 & 0.004209801 & 0.039000128 \\
\hline 2 & $-28 \mathrm{~cm}$ & $-24 \mathrm{~cm}$ & 1800 & 1900 & 0.004007741 & 0.040000125 \\
\hline 2 & $-28 \mathrm{~cm}$ & $-24 \mathrm{~cm}$ & 1900 & 2000 & 0.004253328 & 0.03840013 \\
\hline 2 & $-28 \mathrm{~cm}$ & $-24 \mathrm{~cm}$ & 2000 & 2100 & 0.00440218 & 0.034001324 \\
\hline 2 & $-28 \mathrm{~cm}$ & $-24 \mathrm{~cm}$ & 3000 & 4000 & 0.004377412 & 0.033901327 \\
\hline 2 & $-28 \mathrm{~cm}$ & $-24 \mathrm{~cm}$ & 4000 & 5000 & 0.00443812 & 0.033801331 \\
\hline 2 & $-28 \mathrm{~cm}$ & $-24 \mathrm{~cm}$ & 5000 & 10000 & 0.004463775 & 0.032207608 \\
\hline 3 & $-24 \mathrm{~cm}$ & $-20 \mathrm{~cm}$ & 200 & 300 & 0.000156383 & 0.267300019 \\
\hline 3 & $-24 \mathrm{~cm}$ & $-20 \mathrm{~cm}$ & 300 & 400 & 0.001396224 & 0.08300006 \\
\hline 3 & $-24 \mathrm{~cm}$ & $-20 \mathrm{~cm}$ & 400 & 500 & 0.002632865 & 0.057900086 \\
\hline 3 & $-24 \mathrm{~cm}$ & $-20 \mathrm{~cm}$ & 500 & 600 & 0.002964812 & 0.053100094 \\
\hline 3 & $-24 \mathrm{~cm}$ & $-20 \mathrm{~cm}$ & 600 & 700 & 0.003338586 & 0.049000102 \\
\hline 3 & $-24 \mathrm{~cm}$ & $-20 \mathrm{~cm}$ & 700 & 800 & 0.003505548 & 0.047300106 \\
\hline
\end{tabular}




\begin{tabular}{|c|c|c|c|c|c|c|}
\hline $\begin{array}{l}\text { Segment } \\
\text { number }\end{array}$ & $\begin{array}{l}\text { z } \\
\text { bottom }\end{array}$ & z top & $\begin{array}{l}\text { Min } \\
\text { Energy } \\
(\mathrm{keV})\end{array}$ & $\begin{array}{l}\text { Max } \\
\text { Energy } \\
\text { (keV) }\end{array}$ & pflux/eflux & $\begin{array}{l}\text { Relative } \\
\text { uncertainty }\end{array}$ \\
\hline 3 & $-24 \mathrm{~cm}$ & $-20 \mathrm{~cm}$ & 800 & 900 & 0.003663404 & 0.045700109 \\
\hline 3 & $-24 \mathrm{~cm}$ & $-20 \mathrm{~cm}$ & 900 & 1000 & 0.00377887 & 0.044800112 \\
\hline 3 & $-24 \mathrm{~cm}$ & $-20 \mathrm{~cm}$ & 1000 & 1100 & 0.003957745 & 0.043100116 \\
\hline 3 & $-24 \mathrm{~cm}$ & $-20 \mathrm{~cm}$ & 1100 & 1200 & 0.004051902 & 0.041900119 \\
\hline 3 & $-24 \mathrm{~cm}$ & $-20 \mathrm{~cm}$ & 1200 & 1300 & 0.004386976 & 0.039800126 \\
\hline 3 & $-24 \mathrm{~cm}$ & $-20 \mathrm{~cm}$ & 1300 & 1400 & 0.004184467 & 0.040700123 \\
\hline 3 & $-24 \mathrm{~cm}$ & $-20 \mathrm{~cm}$ & 1400 & 1500 & 0.004284923 & 0.039700126 \\
\hline 3 & $-24 \mathrm{~cm}$ & $-20 \mathrm{~cm}$ & 1500 & 1600 & 0.004395847 & 0.039100128 \\
\hline 3 & $-24 \mathrm{~cm}$ & $-20 \mathrm{~cm}$ & 1600 & 1700 & 0.004462052 & 0.038300131 \\
\hline 3 & $-24 \mathrm{~cm}$ & $-20 \mathrm{~cm}$ & 1700 & 1800 & 0.004337172 & 0.03840013 \\
\hline 3 & $-24 \mathrm{~cm}$ & $-20 \mathrm{~cm}$ & 1800 & 1900 & 0.004115368 & 0.039500127 \\
\hline 3 & $-24 \mathrm{~cm}$ & $-20 \mathrm{~cm}$ & 1900 & 2000 & 0.004415238 & 0.037700133 \\
\hline 3 & $-24 \mathrm{~cm}$ & $-20 \mathrm{~cm}$ & 2000 & 2100 & 0.004533467 & 0.033501343 \\
\hline 3 & $-24 \mathrm{~cm}$ & $-20 \mathrm{~cm}$ & 3000 & 4000 & 0.004512325 & 0.033401347 \\
\hline 3 & $-24 \mathrm{~cm}$ & $-20 \mathrm{~cm}$ & 4000 & 5000 & 0.004616648 & 0.033101359 \\
\hline 3 & $-24 \mathrm{~cm}$ & $-20 \mathrm{~cm}$ & 5000 & 10000 & 0.004630594 & 0.031607752 \\
\hline 4 & $-20 \mathrm{~cm}$ & $-16 \mathrm{~cm}$ & 200 & 300 & 0.000156383 & 0.267300019 \\
\hline
\end{tabular}




\begin{tabular}{|c|c|c|c|c|c|c|}
\hline $\begin{array}{l}\text { Segment } \\
\text { number }\end{array}$ & $\begin{array}{l}\text { z } \\
\text { bottom }\end{array}$ & z top & $\begin{array}{l}\text { Min } \\
\text { Energy } \\
(\mathrm{keV})\end{array}$ & $\begin{array}{l}\text { Max } \\
\text { Energy } \\
\text { (keV) }\end{array}$ & pflux/eflux & $\begin{array}{l}\text { Relative } \\
\text { uncertainty }\end{array}$ \\
\hline 4 & $-20 \mathrm{~cm}$ & $-16 \mathrm{~cm}$ & 300 & 400 & 0.001434742 & 0.081900061 \\
\hline 4 & $-20 \mathrm{~cm}$ & $-16 \mathrm{~cm}$ & 400 & 500 & 0.002730053 & 0.056900088 \\
\hline 4 & $-20 \mathrm{~cm}$ & $-16 \mathrm{~cm}$ & 500 & 600 & 0.003082054 & 0.052100096 \\
\hline 4 & $-20 \mathrm{~cm}$ & $-16 \mathrm{~cm}$ & 600 & 700 & 0.003450958 & 0.048200104 \\
\hline 4 & $-20 \mathrm{~cm}$ & $-16 \mathrm{~cm}$ & 700 & 800 & 0.003615332 & 0.046600107 \\
\hline 4 & $-20 \mathrm{~cm}$ & $-16 \mathrm{~cm}$ & 800 & 900 & 0.003763035 & 0.045100111 \\
\hline 4 & $-20 \mathrm{~cm}$ & $-16 \mathrm{~cm}$ & 900 & 1000 & 0.003915191 & 0.044000114 \\
\hline 4 & $-20 \mathrm{~cm}$ & $-16 \mathrm{~cm}$ & 1000 & 1100 & 0.004162209 & 0.042000119 \\
\hline 4 & $-20 \mathrm{~cm}$ & $-16 \mathrm{~cm}$ & 1100 & 1200 & 0.004200909 & 0.041200121 \\
\hline 4 & $-20 \mathrm{~cm}$ & $-16 \mathrm{~cm}$ & 1200 & 1300 & 0.004581341 & 0.039000128 \\
\hline 4 & $-20 \mathrm{~cm}$ & $-16 \mathrm{~cm}$ & 1300 & 1400 & 0.00440398 & 0.039600126 \\
\hline 4 & $-20 \mathrm{~cm}$ & $-16 \mathrm{~cm}$ & 1400 & 1500 & 0.004440387 & 0.039000128 \\
\hline 4 & $-20 \mathrm{~cm}$ & $-16 \mathrm{~cm}$ & 1500 & 1600 & 0.004562108 & 0.03840013 \\
\hline 4 & $-20 \mathrm{~cm}$ & $-16 \mathrm{~cm}$ & 1600 & 1700 & 0.004637663 & 0.037600133 \\
\hline 4 & $-20 \mathrm{~cm}$ & $-16 \mathrm{~cm}$ & 1700 & 1800 & 0.00457919 & 0.037400134 \\
\hline 4 & $-20 \mathrm{~cm}$ & $-16 \mathrm{~cm}$ & 1800 & 1900 & 0.004317991 & 0.03850013 \\
\hline 4 & $-20 \mathrm{~cm}$ & $-16 \mathrm{~cm}$ & 1900 & 2000 & 0.004539781 & 0.037100135 \\
\hline
\end{tabular}




\begin{tabular}{|c|c|c|c|c|c|c|}
\hline $\begin{array}{l}\text { Segment } \\
\text { number }\end{array}$ & $\begin{array}{l}\text { z } \\
\text { bottom }\end{array}$ & z top & $\begin{array}{l}\text { Min } \\
\text { Energy } \\
(\mathrm{keV})\end{array}$ & $\begin{array}{l}\text { Max } \\
\text { Energy } \\
\text { (keV) }\end{array}$ & pflux/eflux & $\begin{array}{l}\text { Relative } \\
\text { uncertainty }\end{array}$ \\
\hline 4 & $-20 \mathrm{~cm}$ & $-16 \mathrm{~cm}$ & 2000 & 2100 & 0.004609182 & 0.033201355 \\
\hline 4 & $-20 \mathrm{~cm}$ & $-16 \mathrm{~cm}$ & 3000 & 4000 & 0.004717222 & 0.032701376 \\
\hline 4 & $-20 \mathrm{~cm}$ & $-16 \mathrm{~cm}$ & 4000 & 5000 & 0.004735635 & 0.032701376 \\
\hline 4 & $-20 \mathrm{~cm}$ & $-16 \mathrm{~cm}$ & 5000 & 10000 & 0.004833506 & 0.031107877 \\
\hline 5 & $-16 \mathrm{~cm}$ & $-12 \mathrm{~cm}$ & 200 & 300 & 0.000156383 & 0.267300019 \\
\hline 5 & $-16 \mathrm{~cm}$ & $-12 \mathrm{~cm}$ & 300 & 400 & 0.001492518 & 0.080300062 \\
\hline 5 & $-16 \mathrm{~cm}$ & $-12 \mathrm{~cm}$ & 400 & 500 & 0.002836084 & 0.05580009 \\
\hline 5 & $-16 \mathrm{~cm}$ & $-12 \mathrm{~cm}$ & 500 & 600 & 0.003232816 & 0.050900098 \\
\hline 5 & $-16 \mathrm{~cm}$ & $-12 \mathrm{~cm}$ & 600 & 700 & 0.003603443 & 0.047200106 \\
\hline 5 & $-16 \mathrm{~cm}$ & $-12 \mathrm{~cm}$ & 700 & 800 & 0.00374867 & 0.045700109 \\
\hline 5 & $-16 \mathrm{~cm}$ & $-12 \mathrm{~cm}$ & 800 & 900 & 0.003908652 & 0.044300113 \\
\hline 5 & $-16 \mathrm{~cm}$ & $-12 \mathrm{~cm}$ & 900 & 1000 & 0.004059077 & 0.043200116 \\
\hline 5 & $-16 \mathrm{~cm}$ & $-12 \mathrm{~cm}$ & 1000 & 1100 & 0.00431554 & 0.041300121 \\
\hline 5 & $-16 \mathrm{~cm}$ & $-12 \mathrm{~cm}$ & 1100 & 1200 & 0.004413806 & 0.040200124 \\
\hline 5 & $-16 \mathrm{~cm}$ & $-12 \mathrm{~cm}$ & 1200 & 1300 & 0.004817357 & 0.038000132 \\
\hline 5 & $-16 \mathrm{~cm}$ & $-12 \mathrm{~cm}$ & 1300 & 1400 & 0.004602912 & 0.038800129 \\
\hline 5 & $-16 \mathrm{~cm}$ & $-12 \mathrm{~cm}$ & 1400 & 1500 & 0.004582304 & 0.03850013 \\
\hline
\end{tabular}




\begin{tabular}{|c|c|c|c|c|c|c|}
\hline $\begin{array}{l}\text { Segment } \\
\text { number }\end{array}$ & $\begin{array}{l}\text { z } \\
\text { bottom }\end{array}$ & z top & $\begin{array}{l}\text { Min } \\
\text { Energy } \\
(\mathrm{keV})\end{array}$ & $\begin{array}{l}\text { Max } \\
\text { Energy } \\
\text { (keV) }\end{array}$ & pflux/eflux & $\begin{array}{l}\text { Relative } \\
\text { uncertainty }\end{array}$ \\
\hline 5 & $-16 \mathrm{~cm}$ & $-12 \mathrm{~cm}$ & 1500 & 1600 & 0.004754965 & 0.037600133 \\
\hline 5 & $-16 \mathrm{~cm}$ & $-12 \mathrm{~cm}$ & 1600 & 1700 & 0.004735235 & 0.037200134 \\
\hline 5 & $-16 \mathrm{~cm}$ & $-12 \mathrm{~cm}$ & 1700 & 1800 & 0.004732052 & 0.036900136 \\
\hline 5 & $-16 \mathrm{~cm}$ & $-12 \mathrm{~cm}$ & 1800 & 1900 & 0.004495253 & 0.037700133 \\
\hline 5 & $-16 \mathrm{~cm}$ & $-12 \mathrm{~cm}$ & 1900 & 2000 & 0.004689249 & 0.036500137 \\
\hline 5 & $-16 \mathrm{~cm}$ & $-12 \mathrm{~cm}$ & 2000 & 2100 & 0.004790936 & 0.03260138 \\
\hline 5 & $-16 \mathrm{~cm}$ & $-12 \mathrm{~cm}$ & 3000 & 4000 & 0.004867143 & 0.032301393 \\
\hline 5 & $-16 \mathrm{~cm}$ & $-12 \mathrm{~cm}$ & 4000 & 5000 & 0.004924071 & 0.032001406 \\
\hline 5 & $-16 \mathrm{~cm}$ & $-12 \mathrm{~cm}$ & 5000 & 10000 & 0.005009349 & 0.030608005 \\
\hline 6 & $-12 \mathrm{~cm}$ & $-8 \mathrm{~cm}$ & 200 & 300 & 0.000178723 & 0.25000002 \\
\hline 6 & $-12 \mathrm{~cm}$ & $-8 \mathrm{~cm}$ & 300 & 400 & 0.001550293 & 0.078800063 \\
\hline 6 & $-12 \mathrm{~cm}$ & $-8 \mathrm{~cm}$ & 400 & 500 & 0.002871417 & 0.05550009 \\
\hline 6 & $-12 \mathrm{~cm}$ & $-8 \mathrm{~cm}$ & 500 & 600 & 0.003375185 & 0.0498001 \\
\hline 6 & $-12 \mathrm{~cm}$ & $-8 \mathrm{~cm}$ & 600 & 700 & 0.003739868 & 0.046300108 \\
\hline 6 & $-12 \mathrm{~cm}$ & $-8 \mathrm{~cm}$ & 700 & 800 & 0.003889817 & 0.044900111 \\
\hline 6 & $-12 \mathrm{~cm}$ & $-8 \mathrm{~cm}$ & 800 & 900 & 0.004046589 & 0.043500115 \\
\hline 6 & $-12 \mathrm{~cm}$ & $-8 \mathrm{~cm}$ & 900 & 1000 & 0.004202963 & 0.042400118 \\
\hline
\end{tabular}




\begin{tabular}{|c|c|c|c|c|c|c|}
\hline $\begin{array}{l}\text { Segment } \\
\text { number }\end{array}$ & $\begin{array}{l}\text { z } \\
\text { bottom }\end{array}$ & z top & $\begin{array}{l}\text { Min } \\
\text { Energy } \\
(\mathrm{keV})\end{array}$ & $\begin{array}{l}\text { Max } \\
\text { Energy } \\
\text { (keV) }\end{array}$ & pflux/eflux & $\begin{array}{l}\text { Relative } \\
\text { uncertainty }\end{array}$ \\
\hline 6 & $-12 \mathrm{~cm}$ & $-8 \mathrm{~cm}$ & 1000 & 1100 & 0.004476188 & 0.040500123 \\
\hline 6 & $-12 \mathrm{~cm}$ & $-8 \mathrm{~cm}$ & 1100 & 1200 & 0.004584125 & 0.039400127 \\
\hline 6 & $-12 \mathrm{~cm}$ & $-8 \mathrm{~cm}$ & 1200 & 1300 & 0.004970048 & 0.037400134 \\
\hline 6 & $-12 \mathrm{~cm}$ & $-8 \mathrm{~cm}$ & 1300 & 1400 & 0.004733249 & 0.038300131 \\
\hline 6 & $-12 \mathrm{~cm}$ & $-8 \mathrm{~cm}$ & 1400 & 1500 & 0.004778323 & 0.037700133 \\
\hline 6 & $-12 \mathrm{~cm}$ & $-8 \mathrm{~cm}$ & 1500 & 1600 & 0.004874677 & 0.037100135 \\
\hline 6 & $-12 \mathrm{~cm}$ & $-8 \mathrm{~cm}$ & 1600 & 1700 & 0.004943377 & 0.036400137 \\
\hline 6 & $-12 \mathrm{~cm}$ & $-8 \mathrm{~cm}$ & 1700 & 1800 & 0.004993177 & 0.035900139 \\
\hline 6 & $-12 \mathrm{~cm}$ & $-8 \mathrm{~cm}$ & 1800 & 1900 & 0.004704201 & 0.036900136 \\
\hline 6 & $-12 \mathrm{~cm}$ & $-8 \mathrm{~cm}$ & 1900 & 2000 & 0.004813792 & 0.036100139 \\
\hline 6 & $-12 \mathrm{~cm}$ & $-8 \mathrm{~cm}$ & 2000 & 2100 & 0.004942365 & 0.032101402 \\
\hline 6 & $-12 \mathrm{~cm}$ & $-8 \mathrm{~cm}$ & 3000 & 4000 & 0.005081993 & 0.031601424 \\
\hline 6 & $-12 \mathrm{~cm}$ & $-8 \mathrm{~cm}$ & 4000 & 5000 & 0.00504309 & 0.031601424 \\
\hline 6 & $-12 \mathrm{~cm}$ & $-8 \mathrm{~cm}$ & 5000 & 10000 & 0.005261852 & 0.029908193 \\
\hline 7 & $-8 \mathrm{~cm}$ & $-4 \mathrm{~cm}$ & 200 & 300 & 0.000178723 & 0.25000002 \\
\hline 7 & $-8 \mathrm{~cm}$ & $-4 \mathrm{~cm}$ & 300 & 400 & 0.001665844 & 0.076000066 \\
\hline 7 & $-8 \mathrm{~cm}$ & $-4 \mathrm{~cm}$ & 400 & 500 & 0.002986272 & 0.054400092 \\
\hline
\end{tabular}




\begin{tabular}{|c|c|c|c|c|c|c|}
\hline $\begin{array}{l}\text { Segment } \\
\text { number }\end{array}$ & $\begin{array}{l}\text { z } \\
\text { bottom }\end{array}$ & z top & $\begin{array}{l}\text { Min } \\
\text { Energy } \\
(\mathrm{keV})\end{array}$ & $\begin{array}{l}\text { Max } \\
\text { Energy } \\
(\mathrm{keV})\end{array}$ & pflux/eflux & $\begin{array}{l}\text { Relative } \\
\text { uncertainty }\end{array}$ \\
\hline 7 & $-8 \mathrm{~cm}$ & $-4 \mathrm{~cm}$ & 500 & 600 & 0.003500821 & 0.048900102 \\
\hline 7 & $-8 \mathrm{~cm}$ & $-4 \mathrm{~cm}$ & 600 & 700 & 0.003908413 & 0.04530011 \\
\hline 7 & $-8 \mathrm{~cm}$ & $-4 \mathrm{~cm}$ & 700 & 800 & 0.004070211 & 0.043900114 \\
\hline 7 & $-8 \mathrm{~cm}$ & $-4 \mathrm{~cm}$ & 800 & 900 & 0.004230537 & 0.042600117 \\
\hline 7 & $-8 \mathrm{~cm}$ & $-4 \mathrm{~cm}$ & 900 & 1000 & 0.004384724 & 0.04160012 \\
\hline 7 & $-8 \mathrm{~cm}$ & $-4 \mathrm{~cm}$ & 1000 & 1100 & 0.004687947 & 0.039600126 \\
\hline 7 & $-8 \mathrm{~cm}$ & $-4 \mathrm{~cm}$ & 1100 & 1200 & 0.004846669 & 0.038300131 \\
\hline 7 & $-8 \mathrm{~cm}$ & $-4 \mathrm{~cm}$ & 1200 & 1300 & 0.005164413 & 0.036700136 \\
\hline 7 & $-8 \mathrm{~cm}$ & $-4 \mathrm{~cm}$ & 1300 & 1400 & 0.004884166 & 0.037700133 \\
\hline 7 & $-8 \mathrm{~cm}$ & $-4 \mathrm{~cm}$ & 1400 & 1500 & 0.0050081 & 0.036800136 \\
\hline 7 & $-8 \mathrm{~cm}$ & $-4 \mathrm{~cm}$ & 1500 & 1600 & 0.00502763 & 0.036600137 \\
\hline 7 & $-8 \mathrm{~cm}$ & $-4 \mathrm{~cm}$ & 1600 & 1700 & 0.005177552 & 0.035500141 \\
\hline 7 & $-8 \mathrm{~cm}$ & $-4 \mathrm{~cm}$ & 1700 & 1800 & 0.00519698 & 0.035200142 \\
\hline 7 & $-8 \mathrm{~cm}$ & $-4 \mathrm{~cm}$ & 1800 & 1900 & 0.004881483 & 0.036200138 \\
\hline 7 & $-8 \mathrm{~cm}$ & $-4 \mathrm{~cm}$ & 1900 & 2000 & 0.005056676 & 0.035200142 \\
\hline 7 & $-8 \mathrm{~cm}$ & $-4 \mathrm{~cm}$ & 2000 & 2100 & 0.005164498 & 0.031401433 \\
\hline 7 & $-8 \mathrm{~cm}$ & $-4 \mathrm{~cm}$ & 3000 & 4000 & 0.005326859 & 0.030801461 \\
\hline
\end{tabular}




\begin{tabular}{|c|c|c|c|c|c|c|}
\hline $\begin{array}{l}\text { Segment } \\
\text { number }\end{array}$ & $\begin{array}{l}\text { z } \\
\text { bottom }\end{array}$ & z top & $\begin{array}{l}\text { Min } \\
\text { Energy } \\
(\mathrm{keV})\end{array}$ & $\begin{array}{l}\text { Max } \\
\text { Energy } \\
\text { (keV) }\end{array}$ & pflux/eflux & $\begin{array}{l}\text { Relative } \\
\text { uncertainty }\end{array}$ \\
\hline 7 & $-8 \mathrm{~cm}$ & $-4 \mathrm{~cm}$ & 4000 & 5000 & 0.005226572 & 0.031101447 \\
\hline 7 & $-8 \mathrm{~cm}$ & $-4 \mathrm{~cm}$ & 5000 & 10000 & 0.005482781 & 0.029208389 \\
\hline 8 & $-4 \mathrm{~cm}$ & $0 \mathrm{~cm}$ & 200 & 300 & 0.000189894 & 0.242500021 \\
\hline 8 & $-4 \mathrm{~cm}$ & $0 \mathrm{~cm}$ & 300 & 400 & 0.001733248 & 0.074500067 \\
\hline 8 & $-4 \mathrm{~cm}$ & $0 \mathrm{~cm}$ & 400 & 500 & 0.003030459 & 0.054000093 \\
\hline 8 & $-4 \mathrm{~cm}$ & $0 \mathrm{~cm}$ & 500 & 600 & 0.003668317 & 0.047800105 \\
\hline 8 & $-4 \mathrm{~cm}$ & $0 \mathrm{~cm}$ & 600 & 700 & 0.004044838 & 0.044500112 \\
\hline 8 & $-4 \mathrm{~cm}$ & $0 \mathrm{~cm}$ & 700 & 800 & 0.004203524 & 0.043200116 \\
\hline 8 & $-4 \mathrm{~cm}$ & $0 \mathrm{~cm}$ & 800 & 900 & 0.004406804 & 0.04170012 \\
\hline 8 & $-4 \mathrm{~cm}$ & $0 \mathrm{~cm}$ & 900 & 1000 & 0.004574051 & 0.040700123 \\
\hline 8 & $-4 \mathrm{~cm}$ & $0 \mathrm{~cm}$ & 1000 & 1100 & 0.004863209 & 0.038900129 \\
\hline 8 & $-4 \mathrm{~cm}$ & $0 \mathrm{~cm}$ & 1100 & 1200 & 0.005080857 & 0.037400134 \\
\hline 8 & $-4 \mathrm{~cm}$ & $0 \mathrm{~cm}$ & 1200 & 1300 & 0.005351843 & 0.036100139 \\
\hline 8 & $-4 \mathrm{~cm}$ & $0 \mathrm{~cm}$ & 1300 & 1400 & 0.005041937 & 0.037100135 \\
\hline 8 & $-4 \mathrm{~cm}$ & $0 \mathrm{~cm}$ & 1400 & 1500 & 0.005251424 & 0.036000139 \\
\hline 8 & $-4 \mathrm{~cm}$ & $0 \mathrm{~cm}$ & 1500 & 1600 & 0.005247104 & 0.03580014 \\
\hline 8 & $-4 \mathrm{~cm}$ & $0 \mathrm{~cm}$ & 1600 & 1700 & 0.005366179 & 0.034900143 \\
\hline
\end{tabular}




\begin{tabular}{|c|c|c|c|c|c|c|}
\hline $\begin{array}{l}\text { Segment } \\
\text { number }\end{array}$ & $\begin{array}{l}\text { z } \\
\text { bottom }\end{array}$ & z top & $\begin{array}{l}\text { Min } \\
\text { Energy } \\
(\mathrm{keV})\end{array}$ & $\begin{array}{l}\text { Max } \\
\text { Energy } \\
\text { (keV) }\end{array}$ & pflux/eflux & $\begin{array}{l}\text { Relative } \\
\text { uncertainty }\end{array}$ \\
\hline 8 & $-4 \mathrm{~cm}$ & $0 \mathrm{~cm}$ & 1700 & 1800 & 0.005368929 & 0.034600145 \\
\hline 8 & $-4 \mathrm{~cm}$ & $0 \mathrm{~cm}$ & 1800 & 1900 & 0.005065091 & 0.03570014 \\
\hline 8 & $-4 \mathrm{~cm}$ & $0 \mathrm{~cm}$ & 1900 & 2000 & 0.00529332 & 0.034400145 \\
\hline 8 & $-4 \mathrm{~cm}$ & $0 \mathrm{~cm}$ & 2000 & 2100 & 0.005376544 & 0.030701466 \\
\hline 8 & $-4 \mathrm{~cm}$ & $0 \mathrm{~cm}$ & 3000 & 4000 & 0.005516749 & 0.030301485 \\
\hline 8 & $-4 \mathrm{~cm}$ & $0 \mathrm{~cm}$ & 4000 & 5000 & 0.005449716 & 0.030501475 \\
\hline 8 & $-4 \mathrm{~cm}$ & $0 \mathrm{~cm}$ & 5000 & 10000 & 0.005748812 & 0.028508595 \\
\hline 9 & $0 \mathrm{~cm}$ & $4 \mathrm{~cm}$ & 200 & 300 & 0.000212235 & 0.229400022 \\
\hline 9 & $0 \mathrm{~cm}$ & $4 \mathrm{~cm}$ & 300 & 400 & 0.001781395 & 0.073500068 \\
\hline 9 & $0 \mathrm{~cm}$ & $4 \mathrm{~cm}$ & 400 & 500 & 0.003145314 & 0.053000094 \\
\hline 9 & $0 \mathrm{~cm}$ & $4 \mathrm{~cm}$ & 500 & 600 & 0.003844207 & 0.046700107 \\
\hline 9 & $0 \mathrm{~cm}$ & $4 \mathrm{~cm}$ & 600 & 700 & 0.004092994 & 0.044300113 \\
\hline 9 & $0 \mathrm{~cm}$ & $4 \mathrm{~cm}$ & 700 & 800 & 0.00436039 & 0.042400118 \\
\hline 9 & $0 \mathrm{~cm}$ & $4 \mathrm{~cm}$ & 800 & 900 & 0.004606089 & 0.040800123 \\
\hline 9 & $0 \mathrm{~cm}$ & $4 \mathrm{~cm}$ & 900 & 1000 & 0.004717937 & 0.040100125 \\
\hline 9 & $0 \mathrm{~cm}$ & $4 \mathrm{~cm}$ & 1000 & 1100 & 0.00505306 & 0.038200131 \\
\hline 9 & $0 \mathrm{~cm}$ & $4 \mathrm{~cm}$ & 1100 & 1200 & 0.005244063 & 0.036800136 \\
\hline
\end{tabular}




\begin{tabular}{|c|c|c|c|c|c|c|}
\hline $\begin{array}{l}\text { Segment } \\
\text { number }\end{array}$ & $\begin{array}{l}\text { z } \\
\text { bottom }\end{array}$ & z top & $\begin{array}{l}\text { Min } \\
\text { Energy } \\
(\mathrm{keV})\end{array}$ & $\begin{array}{l}\text { Max } \\
\text { Energy } \\
\text { (keV) }\end{array}$ & pflux/eflux & $\begin{array}{l}\text { Relative } \\
\text { uncertainty }\end{array}$ \\
\hline 9 & $0 \mathrm{~cm}$ & $4 \mathrm{~cm}$ & 1200 & 1300 & 0.005504557 & 0.03560014 \\
\hline 9 & $0 \mathrm{~cm}$ & $4 \mathrm{~cm}$ & 1300 & 1400 & 0.005213435 & 0.036500137 \\
\hline 9 & $0 \mathrm{~cm}$ & $4 \mathrm{~cm}$ & 1400 & 1500 & 0.00538659 & 0.035500141 \\
\hline 9 & $0 \mathrm{~cm}$ & $4 \mathrm{~cm}$ & 1500 & 1600 & 0.005459913 & 0.035100142 \\
\hline 9 & $0 \mathrm{~cm}$ & $4 \mathrm{~cm}$ & 1600 & 1700 & 0.005561304 & 0.034300146 \\
\hline 9 & $0 \mathrm{~cm}$ & $4 \mathrm{~cm}$ & 1700 & 1800 & 0.005604584 & 0.033900147 \\
\hline 9 & $0 \mathrm{~cm}$ & $4 \mathrm{~cm}$ & 1800 & 1900 & 0.005299359 & 0.034900143 \\
\hline 9 & $0 \mathrm{~cm}$ & $4 \mathrm{~cm}$ & 1900 & 2000 & 0.005567331 & 0.033600149 \\
\hline 9 & $0 \mathrm{~cm}$ & $4 \mathrm{~cm}$ & 2000 & 2100 & 0.005608764 & 0.030101495 \\
\hline 9 & $0 \mathrm{~cm}$ & $4 \mathrm{~cm}$ & 3000 & 4000 & 0.005721646 & 0.02980151 \\
\hline 9 & $0 \mathrm{~cm}$ & $4 \mathrm{~cm}$ & 4000 & 5000 & 0.005603443 & 0.030101495 \\
\hline 9 & $0 \mathrm{~cm}$ & $4 \mathrm{~cm}$ & 5000 & 10000 & 0.00599681 & 0.028008749 \\
\hline 10 & $4 \mathrm{~cm}$ & $8 \mathrm{~cm}$ & 200 & 300 & 0.000234575 & 0.218200023 \\
\hline 10 & $4 \mathrm{~cm}$ & $8 \mathrm{~cm}$ & 300 & 400 & 0.001858431 & 0.072000069 \\
\hline 10 & $4 \mathrm{~cm}$ & $8 \mathrm{~cm}$ & 400 & 500 & 0.003251342 & 0.052100096 \\
\hline 10 & $4 \mathrm{~cm}$ & $8 \mathrm{~cm}$ & 500 & 600 & 0.004061957 & 0.04540011 \\
\hline 10 & $4 \mathrm{~cm}$ & $8 \mathrm{~cm}$ & 600 & 700 & 0.004293635 & 0.043200116 \\
\hline
\end{tabular}




\begin{tabular}{|c|c|c|c|c|c|c|}
\hline $\begin{array}{l}\text { Segment } \\
\text { number }\end{array}$ & $\begin{array}{l}\text { z } \\
\text { bottom }\end{array}$ & z top & $\begin{array}{l}\text { Min } \\
\text { Energy } \\
\text { (keV) }\end{array}$ & $\begin{array}{l}\text { Max } \\
\text { Energy } \\
\text { (keV) }\end{array}$ & pflux/eflux & $\begin{array}{l}\text { Relative } \\
\text { uncertainty }\end{array}$ \\
\hline 10 & $4 \mathrm{~cm}$ & $8 \mathrm{~cm}$ & 700 & 800 & 0.004509397 & 0.04170012 \\
\hline 10 & $4 \mathrm{~cm}$ & $8 \mathrm{~cm}$ & 800 & 900 & 0.00480535 & 0.039900125 \\
\hline 10 & $4 \mathrm{~cm}$ & $8 \mathrm{~cm}$ & 900 & 1000 & 0.00498299 & 0.039000128 \\
\hline 10 & $4 \mathrm{~cm}$ & $8 \mathrm{~cm}$ & 1000 & 1100 & 0.005184506 & 0.037700133 \\
\hline 10 & $4 \mathrm{~cm}$ & $8 \mathrm{~cm}$ & 1100 & 1200 & 0.00548534 & 0.036000139 \\
\hline 10 & $4 \mathrm{~cm}$ & $8 \mathrm{~cm}$ & 1200 & 1300 & 0.005761398 & 0.034800144 \\
\hline 10 & $4 \mathrm{~cm}$ & $8 \mathrm{~cm}$ & 1300 & 1400 & 0.00545355 & 0.03560014 \\
\hline 10 & $4 \mathrm{~cm}$ & $8 \mathrm{~cm}$ & 1400 & 1500 & 0.00558936 & 0.034900143 \\
\hline 10 & $4 \mathrm{~cm}$ & $8 \mathrm{~cm}$ & 1500 & 1600 & 0.005686009 & 0.034400145 \\
\hline 10 & $4 \mathrm{~cm}$ & $8 \mathrm{~cm}$ & 1600 & 1700 & 0.005788981 & 0.033600149 \\
\hline 10 & $4 \mathrm{~cm}$ & $8 \mathrm{~cm}$ & 1700 & 1800 & 0.005814769 & 0.03330015 \\
\hline 10 & $4 \mathrm{~cm}$ & $8 \mathrm{~cm}$ & 1800 & 1900 & 0.005533608 & 0.034100147 \\
\hline 10 & $4 \mathrm{~cm}$ & $8 \mathrm{~cm}$ & 1900 & 2000 & 0.005760386 & 0.033000152 \\
\hline 10 & $4 \mathrm{~cm}$ & $8 \mathrm{~cm}$ & 2000 & 2100 & 0.005866232 & 0.029501525 \\
\hline 10 & $4 \mathrm{~cm}$ & $8 \mathrm{~cm}$ & 3000 & 4000 & 0.005906543 & 0.029301536 \\
\hline 10 & $4 \mathrm{~cm}$ & $8 \mathrm{~cm}$ & 4000 & 5000 & 0.005821634 & 0.029501525 \\
\hline 10 & $4 \mathrm{~cm}$ & $8 \mathrm{~cm}$ & 5000 & 10000 & 0.006195189 & 0.027508908 \\
\hline
\end{tabular}




\begin{tabular}{|c|c|c|c|c|c|c|}
\hline $\begin{array}{l}\text { Segment } \\
\text { number }\end{array}$ & $\begin{array}{l}\text { z } \\
\text { bottom }\end{array}$ & z top & $\begin{array}{l}\text { Min } \\
\text { Energy } \\
(\mathrm{keV})\end{array}$ & $\begin{array}{l}\text { Max } \\
\text { Energy } \\
\text { (keV) }\end{array}$ & pflux/eflux & $\begin{array}{l}\text { Relative } \\
\text { uncertainty }\end{array}$ \\
\hline 11 & $8 \mathrm{~cm}$ & $12 \mathrm{~cm}$ & 200 & 300 & 0.000234575 & 0.218200023 \\
\hline 11 & $8 \mathrm{~cm}$ & $12 \mathrm{~cm}$ & 300 & 400 & 0.001906577 & 0.07110007 \\
\hline 11 & $8 \mathrm{~cm}$ & $12 \mathrm{~cm}$ & 400 & 500 & 0.003339689 & 0.051400097 \\
\hline 11 & $8 \mathrm{~cm}$ & $12 \mathrm{~cm}$ & 500 & 600 & 0.004170859 & 0.044800112 \\
\hline 11 & $8 \mathrm{~cm}$ & $12 \mathrm{~cm}$ & 600 & 700 & 0.00444612 & 0.042500118 \\
\hline 11 & $8 \mathrm{~cm}$ & $12 \mathrm{~cm}$ & 700 & 800 & 0.004760353 & 0.040600123 \\
\hline 11 & $8 \mathrm{~cm}$ & $12 \mathrm{~cm}$ & 800 & 900 & 0.005027604 & 0.039000128 \\
\hline 11 & $8 \mathrm{~cm}$ & $12 \mathrm{~cm}$ & 900 & 1000 & 0.005179882 & 0.038200131 \\
\hline 11 & $8 \mathrm{~cm}$ & $12 \mathrm{~cm}$ & 1000 & 1100 & 0.005454694 & 0.036700136 \\
\hline 11 & $8 \mathrm{~cm}$ & $12 \mathrm{~cm}$ & 1100 & 1200 & 0.005662748 & 0.035400141 \\
\hline 11 & $8 \mathrm{~cm}$ & $12 \mathrm{~cm}$ & 1200 & 1300 & 0.005941872 & 0.034300146 \\
\hline 11 & $8 \mathrm{~cm}$ & $12 \mathrm{~cm}$ & 1300 & 1400 & 0.005755385 & 0.034700144 \\
\hline 11 & $8 \mathrm{~cm}$ & $12 \mathrm{~cm}$ & 1400 & 1500 & 0.005825911 & 0.034100147 \\
\hline 11 & $8 \mathrm{~cm}$ & $12 \mathrm{~cm}$ & 1500 & 1600 & 0.005885531 & 0.033800148 \\
\hline 11 & $8 \mathrm{~cm}$ & $12 \mathrm{~cm}$ & 1600 & 1700 & 0.006094696 & 0.032800152 \\
\hline 11 & $8 \mathrm{~cm}$ & $12 \mathrm{~cm}$ & 1700 & 1800 & 0.006082257 & 0.032500154 \\
\hline 11 & $8 \mathrm{~cm}$ & $12 \mathrm{~cm}$ & 1800 & 1900 & 0.005767876 & 0.03340015 \\
\hline
\end{tabular}




\begin{tabular}{|c|c|c|c|c|c|c|}
\hline $\begin{array}{l}\text { Segment } \\
\text { number }\end{array}$ & $\begin{array}{l}\text { z } \\
\text { bottom }\end{array}$ & z top & $\begin{array}{l}\text { Min } \\
\text { Energy } \\
\text { (keV) }\end{array}$ & $\begin{array}{l}\text { Max } \\
\text { Energy } \\
\text { (keV) }\end{array}$ & pflux/eflux & $\begin{array}{l}\text { Relative } \\
\text { uncertainty }\end{array}$ \\
\hline 11 & $8 \mathrm{~cm}$ & $12 \mathrm{~cm}$ & 1900 & 2000 & 0.006053081 & 0.032200155 \\
\hline 11 & $8 \mathrm{~cm}$ & $12 \mathrm{~cm}$ & 2000 & 2100 & 0.006073234 & 0.029001552 \\
\hline 11 & $8 \mathrm{~cm}$ & $12 \mathrm{~cm}$ & 3000 & 4000 & 0.006171378 & 0.028701568 \\
\hline 11 & $8 \mathrm{~cm}$ & $12 \mathrm{~cm}$ & 4000 & 5000 & 0.006064625 & 0.029001552 \\
\hline 11 & $8 \mathrm{~cm}$ & $12 \mathrm{~cm}$ & 5000 & 10000 & 0.006420637 & 0.027109039 \\
\hline 12 & $12 \mathrm{~cm}$ & $16 \mathrm{~cm}$ & 200 & 300 & 0.000245746 & 0.213200023 \\
\hline 12 & $12 \mathrm{~cm}$ & $16 \mathrm{~cm}$ & 300 & 400 & 0.002002868 & 0.069300072 \\
\hline 12 & $12 \mathrm{~cm}$ & $16 \mathrm{~cm}$ & 400 & 500 & 0.003525211 & 0.0501001 \\
\hline 12 & $12 \mathrm{~cm}$ & $16 \mathrm{~cm}$ & 500 & 600 & 0.004363482 & 0.043800114 \\
\hline 12 & $12 \mathrm{~cm}$ & $16 \mathrm{~cm}$ & 600 & 700 & 0.004670838 & 0.04150012 \\
\hline 12 & $12 \mathrm{~cm}$ & $16 \mathrm{~cm}$ & 700 & 800 & 0.004956417 & 0.039800126 \\
\hline 12 & $12 \mathrm{~cm}$ & $16 \mathrm{~cm}$ & 800 & 900 & 0.005188559 & 0.03850013 \\
\hline 12 & $12 \mathrm{~cm}$ & $16 \mathrm{~cm}$ & 900 & 1000 & 0.005391928 & 0.037500133 \\
\hline 12 & $12 \mathrm{~cm}$ & $16 \mathrm{~cm}$ & 1000 & 1100 & 0.005644545 & 0.036100139 \\
\hline 12 & $12 \mathrm{~cm}$ & $16 \mathrm{~cm}$ & 1100 & 1200 & 0.005925314 & 0.034600145 \\
\hline 12 & $12 \mathrm{~cm}$ & $16 \mathrm{~cm}$ & 1200 & 1300 & 0.006129302 & 0.033700148 \\
\hline 12 & $12 \mathrm{~cm}$ & $16 \mathrm{~cm}$ & 1300 & 1400 & 0.00596117 & 0.034100147 \\
\hline
\end{tabular}




\begin{tabular}{|c|c|c|c|c|c|c|}
\hline $\begin{array}{l}\text { Segment } \\
\text { number }\end{array}$ & $\begin{array}{l}\text { z } \\
\text { bottom }\end{array}$ & z top & $\begin{array}{l}\text { Min } \\
\text { Energy } \\
\text { (keV) }\end{array}$ & $\begin{array}{l}\text { Max } \\
\text { Energy } \\
\text { (keV) }\end{array}$ & pflux/eflux & $\begin{array}{l}\text { Relative } \\
\text { uncertainty }\end{array}$ \\
\hline 12 & $12 \mathrm{~cm}$ & $16 \mathrm{~cm}$ & 1400 & 1500 & 0.006096242 & 0.03340015 \\
\hline 12 & $12 \mathrm{~cm}$ & $16 \mathrm{~cm}$ & 1500 & 1600 & 0.006144888 & 0.033000152 \\
\hline 12 & $12 \mathrm{~cm}$ & $16 \mathrm{~cm}$ & 1600 & 1700 & 0.006393892 & 0.032000156 \\
\hline 12 & $12 \mathrm{~cm}$ & $16 \mathrm{~cm}$ & 1700 & 1800 & 0.006292442 & 0.032000156 \\
\hline 12 & $12 \mathrm{~cm}$ & $16 \mathrm{~cm}$ & 1800 & 1900 & 0.00605913 & 0.032600153 \\
\hline 12 & $12 \mathrm{~cm}$ & $16 \mathrm{~cm}$ & 1900 & 2000 & 0.0062648 & 0.031600158 \\
\hline 12 & $12 \mathrm{~cm}$ & $16 \mathrm{~cm}$ & 2000 & 2100 & 0.006330703 & 0.028401584 \\
\hline 12 & $12 \mathrm{~cm}$ & $16 \mathrm{~cm}$ & 3000 & 4000 & 0.006446228 & 0.028001607 \\
\hline 12 & $12 \mathrm{~cm}$ & $16 \mathrm{~cm}$ & 4000 & 5000 & 0.006297678 & 0.028501579 \\
\hline 12 & $12 \mathrm{~cm}$ & $16 \mathrm{~cm}$ & 5000 & 10000 & 0.006664131 & 0.026609209 \\
\hline 13 & $16 \mathrm{~cm}$ & $20 \mathrm{~cm}$ & 200 & 300 & 0.000245746 & 0.213200023 \\
\hline 13 & $16 \mathrm{~cm}$ & $20 \mathrm{~cm}$ & 300 & 400 & 0.002070275 & 0.068200073 \\
\hline 13 & $16 \mathrm{~cm}$ & $20 \mathrm{~cm}$ & 400 & 500 & 0.003640093 & 0.049300101 \\
\hline 13 & $16 \mathrm{~cm}$ & $20 \mathrm{~cm}$ & 500 & 600 & 0.004572866 & 0.042800117 \\
\hline 13 & $16 \mathrm{~cm}$ & $20 \mathrm{~cm}$ & 600 & 700 & 0.004895557 & 0.040500123 \\
\hline 13 & $16 \mathrm{~cm}$ & $20 \mathrm{~cm}$ & 700 & 800 & 0.005144621 & 0.039000128 \\
\hline 13 & $16 \mathrm{~cm}$ & $20 \mathrm{~cm}$ & 800 & 900 & 0.005464456 & 0.037600133 \\
\hline
\end{tabular}




\begin{tabular}{|c|c|c|c|c|c|c|}
\hline $\begin{array}{l}\text { Segment } \\
\text { number }\end{array}$ & $\begin{array}{l}\text { z } \\
\text { bottom }\end{array}$ & z top & $\begin{array}{l}\text { Min } \\
\text { Energy } \\
\text { (keV) }\end{array}$ & $\begin{array}{l}\text { Max } \\
\text { Energy } \\
\text { (keV) }\end{array}$ & pflux/eflux & $\begin{array}{l}\text { Relative } \\
\text { uncertainty }\end{array}$ \\
\hline 13 & $16 \mathrm{~cm}$ & $20 \mathrm{~cm}$ & 900 & 1000 & 0.005649415 & 0.036700136 \\
\hline 13 & $16 \mathrm{~cm}$ & $20 \mathrm{~cm}$ & 1000 & 1100 & 0.005870917 & 0.035400141 \\
\hline 13 & $16 \mathrm{~cm}$ & $20 \mathrm{~cm}$ & 1100 & 1200 & 0.006109811 & 0.034100147 \\
\hline 13 & $16 \mathrm{~cm}$ & $20 \mathrm{~cm}$ & 1200 & 1300 & 0.006427772 & 0.032900152 \\
\hline 13 & $16 \mathrm{~cm}$ & $20 \mathrm{~cm}$ & 1300 & 1400 & 0.006242425 & 0.03330015 \\
\hline 13 & $16 \mathrm{~cm}$ & $20 \mathrm{~cm}$ & 1400 & 1500 & 0.006420674 & 0.032500154 \\
\hline 13 & $16 \mathrm{~cm}$ & $20 \mathrm{~cm}$ & 1500 & 1600 & 0.006497362 & 0.032100156 \\
\hline 13 & $16 \mathrm{~cm}$ & $20 \mathrm{~cm}$ & 1600 & 1700 & 0.006654079 & 0.031400159 \\
\hline 13 & $16 \mathrm{~cm}$ & $20 \mathrm{~cm}$ & 1700 & 1800 & 0.006579038 & 0.03130016 \\
\hline 13 & $16 \mathrm{~cm}$ & $20 \mathrm{~cm}$ & 1800 & 1900 & 0.006331389 & 0.031900157 \\
\hline 13 & $16 \mathrm{~cm}$ & $20 \mathrm{~cm}$ & 1900 & 2000 & 0.00653883 & 0.031000161 \\
\hline 13 & $16 \mathrm{~cm}$ & $20 \mathrm{~cm}$ & 2000 & 2100 & 0.00662855 & 0.027701625 \\
\hline 13 & $16 \mathrm{~cm}$ & $20 \mathrm{~cm}$ & 3000 & 4000 & 0.00668607 & 0.02760163 \\
\hline 13 & $16 \mathrm{~cm}$ & $20 \mathrm{~cm}$ & 4000 & 5000 & 0.006585316 & 0.027901613 \\
\hline 13 & $16 \mathrm{~cm}$ & $20 \mathrm{~cm}$ & 5000 & 10000 & 0.00693917 & 0.026109385 \\
\hline 14 & $20 \mathrm{~cm}$ & $24 \mathrm{~cm}$ & 200 & 300 & 0.000279257 & 0.200000025 \\
\hline 14 & $20 \mathrm{~cm}$ & $24 \mathrm{~cm}$ & 300 & 400 & 0.002205086 & 0.066100076 \\
\hline
\end{tabular}




\begin{tabular}{|c|c|c|c|c|c|c|}
\hline $\begin{array}{l}\text { Segment } \\
\text { number }\end{array}$ & $\begin{array}{l}\text { z } \\
\text { bottom }\end{array}$ & z top & $\begin{array}{l}\text { Min } \\
\text { Energy } \\
(\mathrm{keV})\end{array}$ & $\begin{array}{l}\text { Max } \\
\text { Energy } \\
\text { (keV) }\end{array}$ & pflux/eflux & $\begin{array}{l}\text { Relative } \\
\text { uncertainty }\end{array}$ \\
\hline 14 & $20 \mathrm{~cm}$ & $24 \mathrm{~cm}$ & 400 & 500 & 0.003781455 & 0.048300104 \\
\hline 14 & $20 \mathrm{~cm}$ & $24 \mathrm{~cm}$ & 500 & 600 & 0.004715235 & 0.042100119 \\
\hline 14 & $20 \mathrm{~cm}$ & $24 \mathrm{~cm}$ & 600 & 700 & 0.005128294 & 0.039600126 \\
\hline 14 & $20 \mathrm{~cm}$ & $24 \mathrm{~cm}$ & 700 & 800 & 0.00533285 & 0.038300131 \\
\hline 14 & $20 \mathrm{~cm}$ & $24 \mathrm{~cm}$ & 800 & 900 & 0.005809359 & 0.036400137 \\
\hline 14 & $20 \mathrm{~cm}$ & $24 \mathrm{~cm}$ & 900 & 1000 & 0.005846307 & 0.036000139 \\
\hline 14 & $20 \mathrm{~cm}$ & $24 \mathrm{~cm}$ & 1000 & 1100 & 0.006097288 & 0.034800144 \\
\hline 14 & $20 \mathrm{~cm}$ & $24 \mathrm{~cm}$ & 1100 & 1200 & 0.006414957 & 0.03330015 \\
\hline 14 & $20 \mathrm{~cm}$ & $24 \mathrm{~cm}$ & 1200 & 1300 & 0.006691548 & 0.032300155 \\
\hline 14 & $20 \mathrm{~cm}$ & $24 \mathrm{~cm}$ & 1300 & 1400 & 0.00652368 & 0.032600153 \\
\hline 14 & $20 \mathrm{~cm}$ & $24 \mathrm{~cm}$ & 1400 & 1500 & 0.006623423 & 0.032000156 \\
\hline 14 & $20 \mathrm{~cm}$ & $24 \mathrm{~cm}$ & 1500 & 1600 & 0.006716836 & 0.031600158 \\
\hline 14 & $20 \mathrm{~cm}$ & $24 \mathrm{~cm}$ & 1600 & 1700 & 0.006940279 & 0.030700163 \\
\hline 14 & $20 \mathrm{~cm}$ & $24 \mathrm{~cm}$ & 1700 & 1800 & 0.006821056 & 0.030700163 \\
\hline 14 & $20 \mathrm{~cm}$ & $24 \mathrm{~cm}$ & 1800 & 1900 & 0.006692279 & 0.031000161 \\
\hline 14 & $20 \mathrm{~cm}$ & $24 \mathrm{~cm}$ & 1900 & 2000 & 0.006868872 & 0.030200166 \\
\hline 14 & $20 \mathrm{~cm}$ & $24 \mathrm{~cm}$ & 2000 & 2100 & 0.00688605 & 0.027201654 \\
\hline
\end{tabular}




\begin{tabular}{|c|c|c|c|c|c|c|}
\hline $\begin{array}{l}\text { Segment } \\
\text { number }\end{array}$ & $\begin{array}{l}\text { z } \\
\text { bottom }\end{array}$ & z top & $\begin{array}{l}\text { Min } \\
\text { Energy } \\
\text { (keV) }\end{array}$ & $\begin{array}{l}\text { Max } \\
\text { Energy } \\
\text { (keV) }\end{array}$ & pflux/eflux & $\begin{array}{l}\text { Relative } \\
\text { uncertainty }\end{array}$ \\
\hline 14 & $20 \mathrm{~cm}$ & $24 \mathrm{~cm}$ & 3000 & 4000 & 0.00696092 & 0.027001667 \\
\hline 14 & $20 \mathrm{~cm}$ & $24 \mathrm{~cm}$ & 4000 & 5000 & 0.006962188 & 0.02710166 \\
\hline 14 & $20 \mathrm{~cm}$ & $24 \mathrm{~cm}$ & 5000 & 10000 & 0.007241265 & 0.025609569 \\
\hline 15 & $24 \mathrm{~cm}$ & $28 \mathrm{~cm}$ & 200 & 300 & 0.000223424 & 0.707100064 \\
\hline 15 & $24 \mathrm{~cm}$ & $28 \mathrm{~cm}$ & 300 & 400 & 0.002600025 & 0.192400234 \\
\hline 15 & $24 \mathrm{~cm}$ & $28 \mathrm{~cm}$ & 400 & 500 & 0.004594265 & 0.138700324 \\
\hline 15 & $24 \mathrm{~cm}$ & $28 \mathrm{~cm}$ & 500 & 600 & 0.00485896 & 0.131300343 \\
\hline 15 & $24 \mathrm{~cm}$ & $28 \mathrm{~cm}$ & 600 & 700 & 0.004735437 & 0.130200346 \\
\hline 15 & $24 \mathrm{~cm}$ & $28 \mathrm{~cm}$ & 700 & 800 & 0.00502184 & 0.12500036 \\
\hline 15 & $24 \mathrm{~cm}$ & $28 \mathrm{~cm}$ & 800 & 900 & 0.006052566 & 0.1125004 \\
\hline 15 & $24 \mathrm{~cm}$ & $28 \mathrm{~cm}$ & 900 & 1000 & 0.007194683 & 0.102600439 \\
\hline 15 & $24 \mathrm{~cm}$ & $28 \mathrm{~cm}$ & 1000 & 1100 & 0.005036437 & 0.120400374 \\
\hline 15 & $24 \mathrm{~cm}$ & $28 \mathrm{~cm}$ & 1100 & 1200 & 0.007098227 & 0.101000446 \\
\hline 15 & $24 \mathrm{~cm}$ & $28 \mathrm{~cm}$ & 1200 & 1300 & 0.007430937 & 0.097600461 \\
\hline 15 & $24 \mathrm{~cm}$ & $28 \mathrm{~cm}$ & 1300 & 1400 & 0.007132549 & 0.098100459 \\
\hline 15 & $24 \mathrm{~cm}$ & $28 \mathrm{~cm}$ & 1400 & 1500 & 0.00730162 & 0.096200468 \\
\hline 15 & $24 \mathrm{~cm}$ & $28 \mathrm{~cm}$ & 1500 & 1600 & 0.006453902 & 0.101500443 \\
\hline
\end{tabular}




\begin{tabular}{|c|c|c|c|c|c|c|}
\hline $\begin{array}{l}\text { Segment } \\
\text { number }\end{array}$ & $\begin{array}{l}\text { z } \\
\text { bottom }\end{array}$ & z top & $\begin{array}{l}\text { Min } \\
\text { Energy } \\
(\mathrm{keV})\end{array}$ & $\begin{array}{l}\text { Max } \\
\text { Energy } \\
\text { (keV) }\end{array}$ & pflux/eflux & $\begin{array}{l}\text { Relative } \\
\text { uncertainty }\end{array}$ \\
\hline 15 & $24 \mathrm{~cm}$ & $28 \mathrm{~cm}$ & 1600 & 1700 & 0.006182954 & 0.102600439 \\
\hline 15 & $24 \mathrm{~cm}$ & $28 \mathrm{~cm}$ & 1700 & 1800 & 0.007324956 & 0.094100478 \\
\hline 15 & $24 \mathrm{~cm}$ & $28 \mathrm{~cm}$ & 1800 & 1900 & 0.00683995 & 0.097100463 \\
\hline 15 & $24 \mathrm{~cm}$ & $28 \mathrm{~cm}$ & 1900 & 2000 & 0.00672575 & 0.096200468 \\
\hline 15 & $24 \mathrm{~cm}$ & $28 \mathrm{~cm}$ & 2000 & 2100 & 0.007613388 & 0.036602186 \\
\hline 15 & $24 \mathrm{~cm}$ & $28 \mathrm{~cm}$ & 3000 & 4000 & 0.007164895 & 0.037602128 \\
\hline 15 & $24 \mathrm{~cm}$ & $28 \mathrm{~cm}$ & 4000 & 5000 & 0.007208067 & 0.037602128 \\
\hline 15 & $24 \mathrm{~cm}$ & $28 \mathrm{~cm}$ & 5000 & 10000 & 0.007538854 & 0.025109759 \\
\hline 16 & $28 \mathrm{~cm}$ & $29 \mathrm{~cm}$ & 200 & 300 & 0.000223424 & 0.707100064 \\
\hline 16 & $28 \mathrm{~cm}$ & $29 \mathrm{~cm}$ & 300 & 400 & 0.002600025 & 0.192400234 \\
\hline 16 & $28 \mathrm{~cm}$ & $29 \mathrm{~cm}$ & 400 & 500 & 0.004594265 & 0.138700324 \\
\hline 16 & $28 \mathrm{~cm}$ & $29 \mathrm{~cm}$ & 500 & 600 & 0.00485896 & 0.131300343 \\
\hline 16 & $28 \mathrm{~cm}$ & $29 \mathrm{~cm}$ & 600 & 700 & 0.004735437 & 0.130200346 \\
\hline 16 & $28 \mathrm{~cm}$ & $29 \mathrm{~cm}$ & 700 & 800 & 0.00502184 & 0.12500036 \\
\hline 16 & $28 \mathrm{~cm}$ & $29 \mathrm{~cm}$ & 800 & 900 & 0.006052566 & 0.1125004 \\
\hline 16 & $28 \mathrm{~cm}$ & $29 \mathrm{~cm}$ & 900 & 1000 & 0.007194683 & 0.102600439 \\
\hline 16 & $28 \mathrm{~cm}$ & $29 \mathrm{~cm}$ & 1000 & 1100 & 0.005036437 & 0.120400374 \\
\hline
\end{tabular}




\begin{tabular}{|c|c|c|c|c|c|c|}
\hline $\begin{array}{l}\text { Segment } \\
\text { number }\end{array}$ & $\begin{array}{l}\text { z } \\
\text { bottom }\end{array}$ & z top & $\begin{array}{l}\text { Min } \\
\text { Energy } \\
\text { (keV) }\end{array}$ & $\begin{array}{l}\text { Max } \\
\text { Energy } \\
\text { (keV) }\end{array}$ & pflux/eflux & $\begin{array}{l}\text { Relative } \\
\text { uncertainty }\end{array}$ \\
\hline 16 & $28 \mathrm{~cm}$ & $29 \mathrm{~cm}$ & 1100 & 1200 & 0.007098227 & 0.101000446 \\
\hline 16 & $28 \mathrm{~cm}$ & $29 \mathrm{~cm}$ & 1200 & 1300 & 0.007430937 & 0.097600461 \\
\hline 16 & $28 \mathrm{~cm}$ & $29 \mathrm{~cm}$ & 1300 & 1400 & 0.007132549 & 0.098100459 \\
\hline 16 & $28 \mathrm{~cm}$ & $29 \mathrm{~cm}$ & 1400 & 1500 & 0.00730162 & 0.096200468 \\
\hline 16 & $28 \mathrm{~cm}$ & $29 \mathrm{~cm}$ & 1500 & 1600 & 0.006453902 & 0.101500443 \\
\hline 16 & $28 \mathrm{~cm}$ & $29 \mathrm{~cm}$ & 1600 & 1700 & 0.006182954 & 0.102600439 \\
\hline 16 & $28 \mathrm{~cm}$ & $29 \mathrm{~cm}$ & 1700 & 1800 & 0.007324956 & 0.094100478 \\
\hline 16 & $28 \mathrm{~cm}$ & $29 \mathrm{~cm}$ & 1800 & 1900 & 0.00683995 & 0.097100463 \\
\hline 16 & $28 \mathrm{~cm}$ & $29 \mathrm{~cm}$ & 1900 & 2000 & 0.00672575 & 0.096200468 \\
\hline 16 & $28 \mathrm{~cm}$ & $29 \mathrm{~cm}$ & 2000 & 2100 & 0.007613388 & 0.036602186 \\
\hline 16 & $28 \mathrm{~cm}$ & $29 \mathrm{~cm}$ & 3000 & 4000 & 0.007164895 & 0.037602128 \\
\hline 16 & $28 \mathrm{~cm}$ & $29 \mathrm{~cm}$ & 4000 & 5000 & 0.007208067 & 0.037602128 \\
\hline 16 & $28 \mathrm{~cm}$ & $29 \mathrm{~cm}$ & 5000 & 10000 & 0.007538854 & 0.025109759 \\
\hline
\end{tabular}




\section{APPENDIX B}

\section{CERENKOV MODELING IN MCNP}

This appendix explains the process of modeling Cerenkov photons using MCNP that is used in this dissertation. There is some redundancy here with different portions of the dissertation, but here the whole process is collected in one place. It covers how MCNP models Cerenkov production, how the Cerenkov production is used in the dissertation, how the electron flux to Cerenkov flux response function is generated with a simpler model and how the response function is used to calculate the Cerenkov flux above the core.

MCNP 6.1.1 beta has added the capability to track photons down to $1 \mathrm{eV}$ of energy (in the infrared range), to account for refraction in materials and to produce and transport Cerenkov radiation. This feature has been verified by Los Alamos National Lab to produce Cerenkov photons in a way that is consistent with other modeling software [44]. The index of refraction of a material is added in the material card, using either refi, refc or refs for a constant, Cauchy or Sellmeier treatment of refraction [43]. When Cerenkov photons are being produced in this research effort, the Sellmeier coefficients are used with an addition to the material card for water of " refs=.56840 5.1018e-3 $1.7262 \mathrm{e}-11.8211 \mathrm{e}-22.0862 \mathrm{e}-$ 2 2.6207e-2". The Sellmeier treatment of refraction has greater agreement with measurements over a wider range of wavelengths than the simplified Cauchy equation or the constant approximation.

If Cerenkov production is turned on in MCNP, electrons travelling through a medium with a refractive index input will produce Cerenkov photons if the electron's 
energy is high enough that its velocity exceeds the phase velocity of light for some wavelength that is being tracked. This is between 200 and $300 \mathrm{keV}$ for water modeled using the Sellmeier equation. Each electron can produce a large number of Cerenkov photons, as the photons have energies below $10 \mathrm{eV}$, while the electrons have energies above $200 \mathrm{keV}$, a factor of 20,000 greater. The Cerenkov photons produced are then transported through the geometry in MCNP in a similar manner to other particles, with some differences at material boundaries due to refraction.

There have been some issues with refraction at boundaries and the transport of visible light through opaque materials that do not have refractive indices specified (such as cladding and fuel). Some tests using Cerenkov production in the whole core resulted in prohibitively long code runtimes and non-physical results caused by light travelling through fuel, cladding and other materials without any appreciable attenuation. Due to these issues and the high computational cost of visible photon transport, the production and transport of Cerenkov photons used in this research effort was limited to very simple models. The simple Cerenkov models are used to produce response functions for use in the whole core model.

The method of simulation aims to measure the Cerenkov radiation at a plane $2 \mathrm{~m}$ above the core midplane, with a separate measurement above each of 81 coolant channels observed. The Cerenkov measured above a channel is restricted to Cerenkov photons with energies corresponding to visible light (1.77 eV to $3.17 \mathrm{eV})$, travelling in a direction within a $\mathrm{mu}=.99995$ cone of the $+\mathrm{z}$ direction to only account for visible light that is coming from the channel. To obtain the Cerenkov response above a single channel, the spatial and 
energy dependent electron flux within a $1 \mathrm{~cm}$ radius cylindrical region occupying most of the channel is tallied. The electron flux within the channel is then multiplied by a response function to obtain the total Cerenkov observed above the channel.

The response functions are generated using a simplified model consisting of only a $1 \mathrm{~cm}$ radius cylinder of water extending from the bottom of the coolant channel $(\mathrm{z}=-$ $29 \mathrm{~cm})$ to the Cerenkov tallying plane above the core $(\mathrm{z}=200 \mathrm{~cm})$. In the model, the cylinder of water is surrounded by more water with an importance of 0 instead of a void to prevent internal reflection caused by the differing refractive indices. This effectively kills any particle that leaves the cylinder of water. The coolant channel is divided into 16 axial segments, most of which are $4 \mathrm{~cm}$ tall. The electron energy spectrum is divided into 22 energy bins, ranging from $200 \mathrm{keV}$ to $10 \mathrm{MeV}$.

To obtain the response function for electrons within a bin, a model is run that only includes electrons within that bin. For example, for spatial segment 12, extending from z $=12 \mathrm{~cm}$ to $\mathrm{z}=16 \mathrm{~cm}$ and the $5^{\text {th }}$ electron energy bin ranging from $600 \mathrm{keV}$ to $700 \mathrm{keV}$, an MCNP model of the cylinder of water is run with electrons with a close to uniform distribution of energy between 600 and $700 \mathrm{keV}$ in the spatial region between $12 \mathrm{~cm}$ and $16 \mathrm{~cm}$. All regions outside of this segment have an electron importance of zero, so no electrons will leave the segment. The electron energy cutoff is set to the minimum of the energy bin, in this case $600 \mathrm{keV}$, so that electrons dropping below this energy are killed. The electrons are spawned homogenously within the segment with an initial energy distribution slanted towards the top of the energy bin that results in a close to uniform energy distribution within the segment, because electrons spawned with energies near the 
top of the bin will in most cases also exist as electrons in the lower portion of the bin as they slow down, before being killed by the minimum energy cutoff. This distribution for each energy bin is produced and checked empirically by using 10 sub bins equally distributed through the energy range, and in most cases was a linear function producing more near the top than the bottom. Thus electrons within the spatial and energy bin are being transported in the column of water, producing Cerenkov photons within the spatial segment. These Cerenkov photons are transported through the water, either being killed upon reaching the edge of the cylinder or reaching the top of the cylinder to cross the tallying plane at $\mathrm{z}=2 \mathrm{~m}$. This tally is the number of Cerenkov photons crossing the plane that were produced by the electrons within the segment, using an F2 type surface tally; no other photons are present. The electron flux within the bin is also tallied using an F4 volume flux tally, and the result of the Cerenkov tally above the core is divided by the bin's total electron flux tally. This gives a ratio for the flux of Cerenkov photons crossing the tallying plane directly above a channel produced per unit path length of electrons within the $1 \mathrm{~cm}$ cylinder in the channel between $\mathrm{z}=12$ and $\mathrm{z}=16 \mathrm{~cm}$ with energies between $600 \mathrm{keV}$ and $700 \mathrm{keV}$, referred to as the Pflux/Eflux for that bin. This single value is a component of the overall response function. In a separate scenario, the electron flux in that spatial/energy bin can then be multiplied by this value to obtain its contribution to the Cerenkov flux above that channel.

The previously mentioned process is done for every spatial and energy bin to obtain a correlation factor relating the Cerenkov above the core to the electron flux in a segment of the channel for every bin. This list of semi empirical correlation factors is the 
response function used to convert the electron flux within a channel into the Cerenkov flux above the channel. The list of factors can be seen in Appendix A.

To obtain the total Cerenkov flux estimate above a channel without transporting Cerenkov photons, an electron tally and the response function are used. The MCNP run uses mode NPE to trace neutrons, photons and electrons, but it keep the energy cutoff for both electrons and photons at $100 \mathrm{keV}$. An electron tally within the $1 \mathrm{~cm}$ radius cylindrical region filling most of the coolant channel is implemented. The electron tally is segmented into 16 segments and has energy binning for 22 energy bins, corresponding to the segments and energy bins of the response function. Then the electron flux measured for each segment and energy bin is multiplied by its correlation factor from the response function to obtain its contribution to the total Cerenkov flux. Each contribution is then added together to obtain the total Cerenkov flux directly above that coolant channel produced by all electrons within the cylindrical region of the coolant channel tallied.

An MCNP run for a geometry consisting of only 4 pins tracking Cerenkov radiation explicitly was performed to test this, and it was found that the electron flux tally with response function method accounted for $92 \%$ of the total tallied Cerenkov flux above the channel coming from the channel. The other $\sim 8 \%$ comes from the regions of the coolant channel not included within the cylinder.

For models of the entire reactor core, 81 coolant channels are observed, resulting in 81 electron flux tallies, one for each coolant channel in the region covered by the flux map. These tallies have all the same segmentation and energy bins, and the response 
function is applied to each channel's tally to obtain the Cerenkov above that channel. All 81 Cerenkov fluxes can then be plotted as a 2D Cerenkov map. 


\section{APPENDIX C}

\section{SCRIPTS USED FOR POST PROCESSING AND PLOTTING}

The python scripts used for the mass production of input decks, generation of the correlation factors and the post processing of MCNP outputs are shown in this appendix. Script editing was done using Notepad ++. There are some text wrapping issues with porting the scripts into this document; however, if one wishes to use these scripts as a reference in generating similar programs, it should be possible to copy them from this document and paste them correctly into another program.

The first script shown here is one of a set of four scripts used to generate the many input decks run for the production of the correlation factors as described in section 2.3 of the document. This script is the second of four, where each one covers a different energy range. This one covers the $1 \mathrm{MeV}$ to $2 \mathrm{MeV}$ energy range. What it does is it takes a base input file and modifies a few lines to create multiple new decks that run electrons in different spatial and energy bins. It also creates a batch file to run all the input decks created sequentially with a single command.

Script: Input_Creater.py:

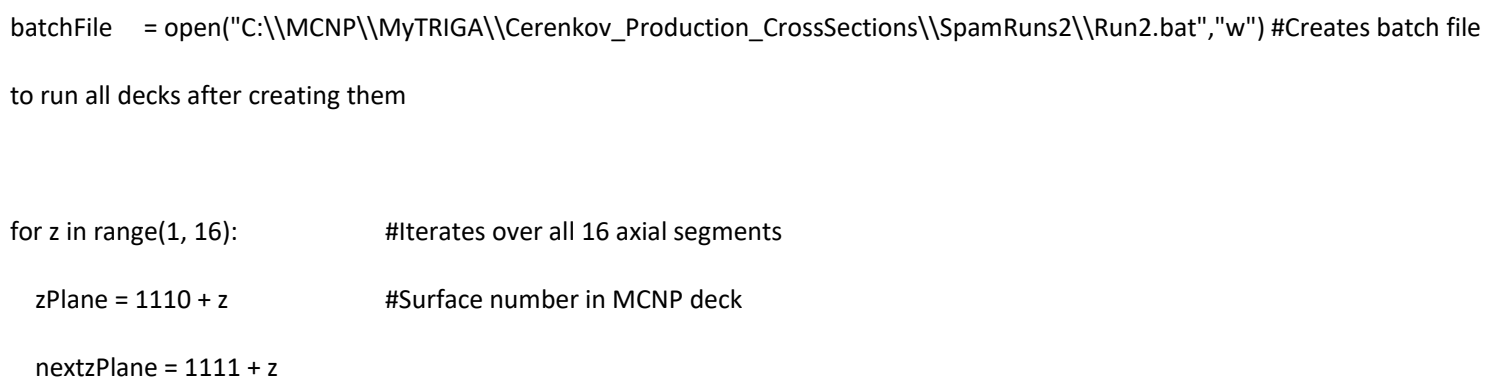




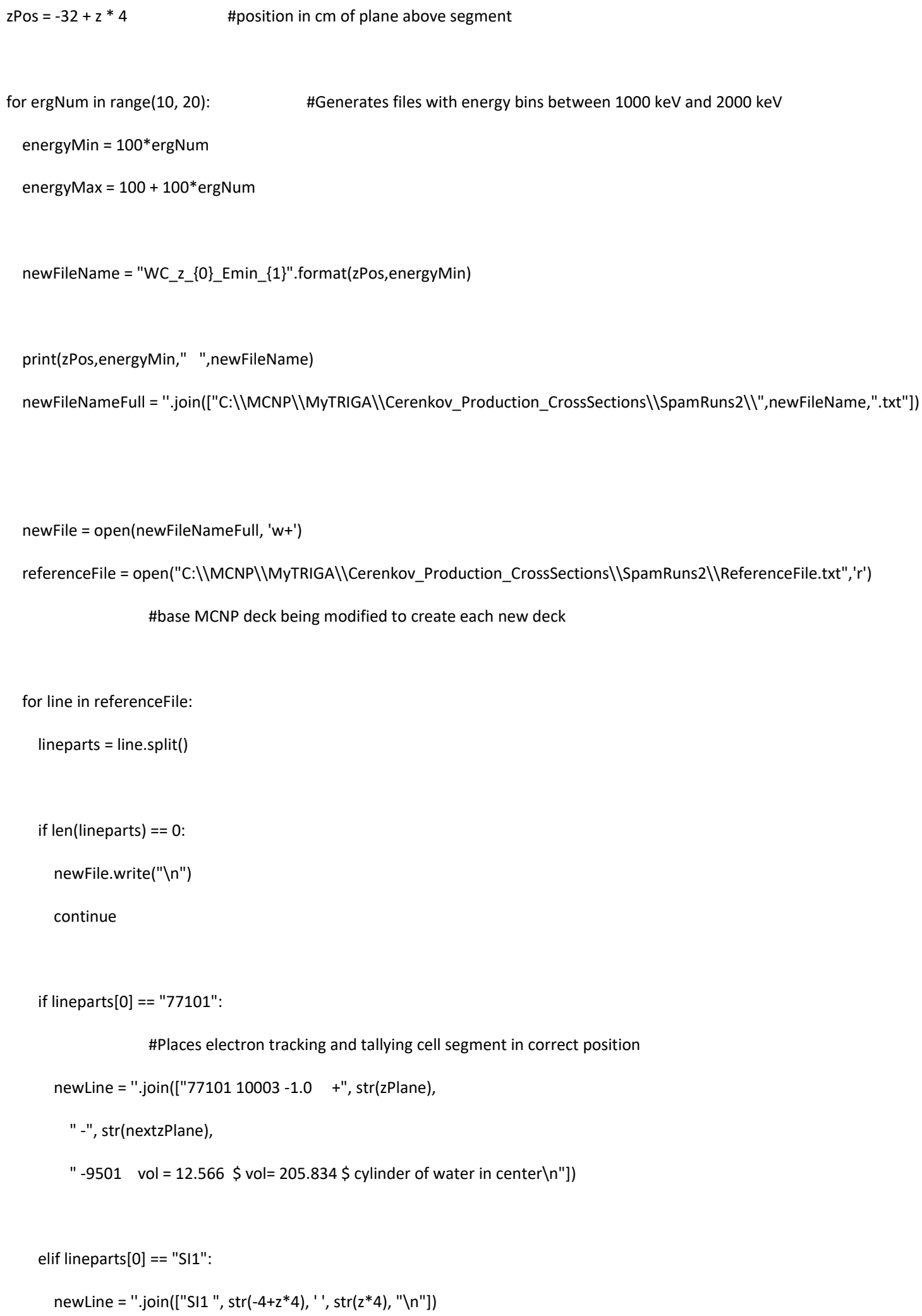




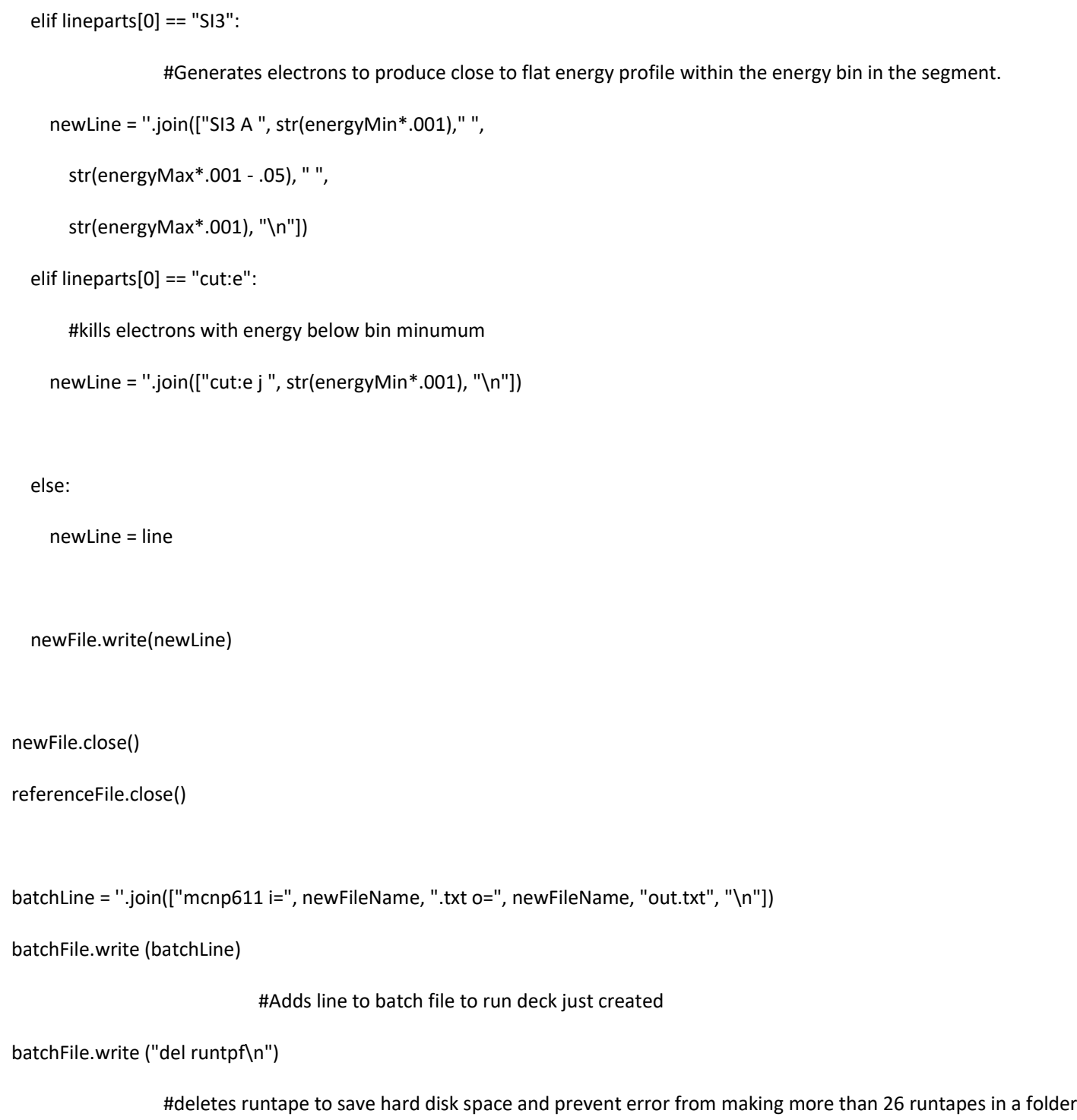

The second script is used in the generation of the electron to Cerenkov flux correlations. This script reads through all the output files generated by MCNP after running all of the decks produced with the previous set of scripts. It takes the electron flux 
with uncertainty from within the segment and energy bin for that output deck, and records it in a text file with the $\mathrm{z}$ position of the segment, and the min and max energy of the bin.. It also does this for the visible Cerenkov flux detected $2 \mathrm{~m}$ above the core within the specified angle bin, creating a second text file. A third text file is generated that has the ratio of the Cerenkov flux to the electron flux, along with its propagated absolute uncertainty. This ratio is the correlation factor used for each segment and energy bin. The usefulness of this script is that it collects all of the correlation factors into a single text file that can then be used elsewhere. The electron and photon flux text files were only used for checking consistency.

Second script: Output_Extractor2.py:

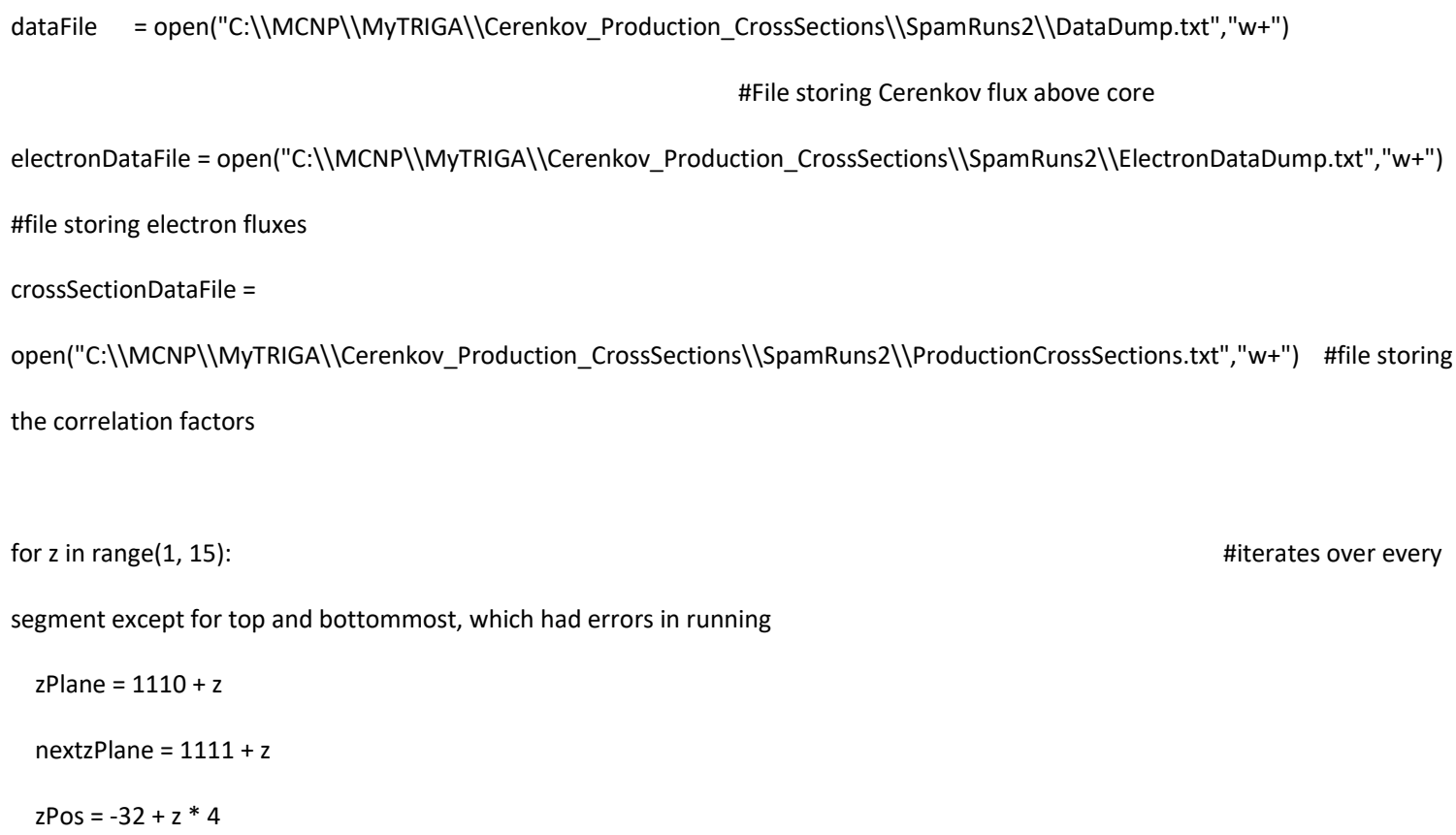


for ergNum in range(1, 24):

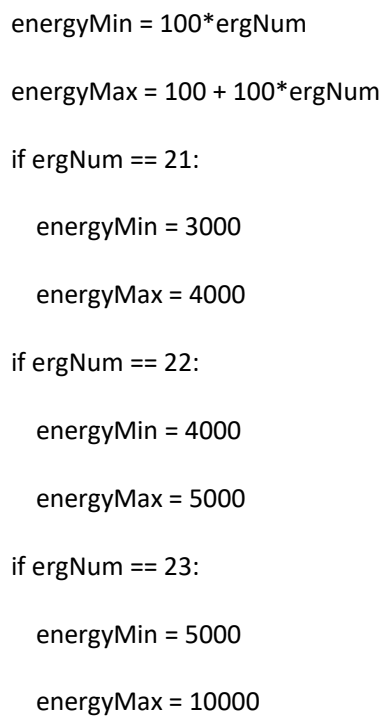




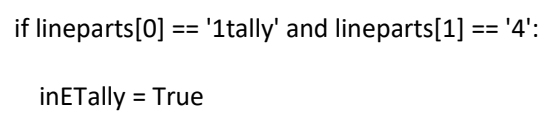

outputFile.close() \#Closes MCNP output file so that next one can be opened 


\section{End second script}

The third script is used to extract the electron flux data from the MCNP run of the whole core. This deck takes the electron fluxes and uses the correlation factors to obtain the total Cerenkov flux above each channel. It can be altered to use an offset viewpoint in addition to the direct view. It also has the ability to simulate a blockage of a coolant channel by inputting the channel, block location and block amount. The Cerenkov and flux tilts for each channel can then be plotted in 2D color map plots. This script can also extract data from two output files and compare the resulting Cerenkov fluxes and flux tilts. Absolute and relative difference plots of the differences in the Cerenkov flux or flux tilt for each channel can be produced.

Manual modification to the script is done between the lines of

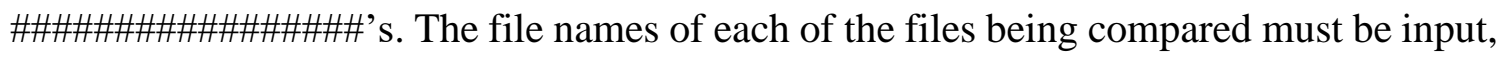
as well as a shorter name/description to be placed in the plots. The options for which channel is to be blocked (if any) in each case along with the position and amount of the block are also input.

Third script: TallyExtractor.py:

import numpy as $\mathrm{np}$ 


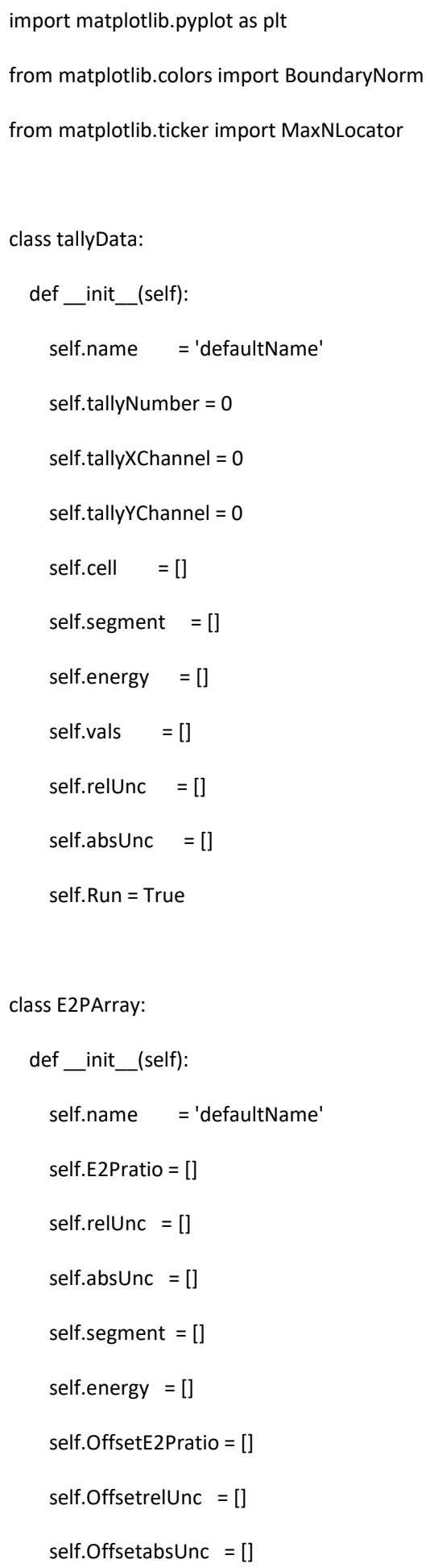




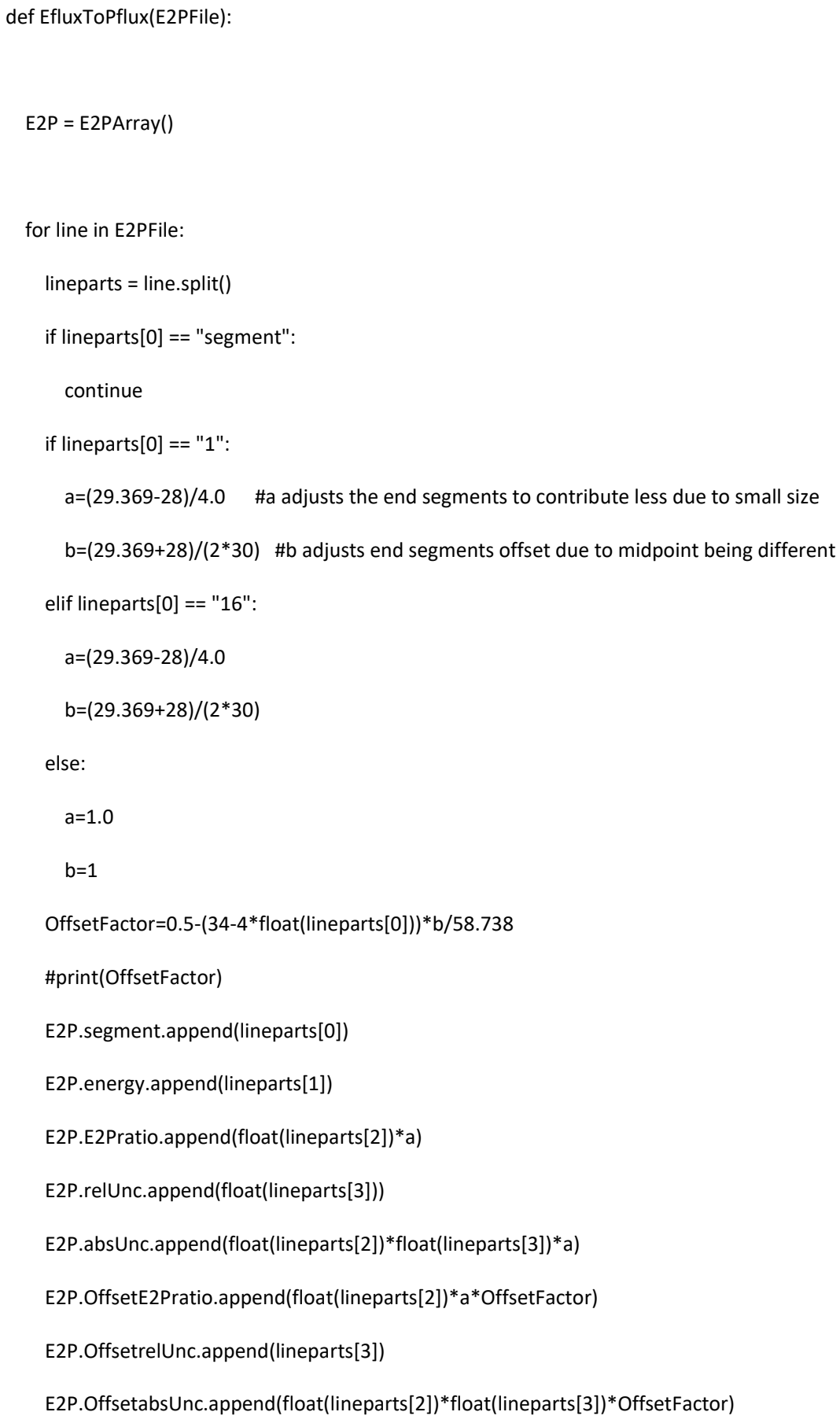

\# for $\mathrm{i}$ in range(len(E2P.E2Pratio)): 
$\operatorname{return}(\mathrm{E} 2 \mathrm{P})$

def extractTallies(file):

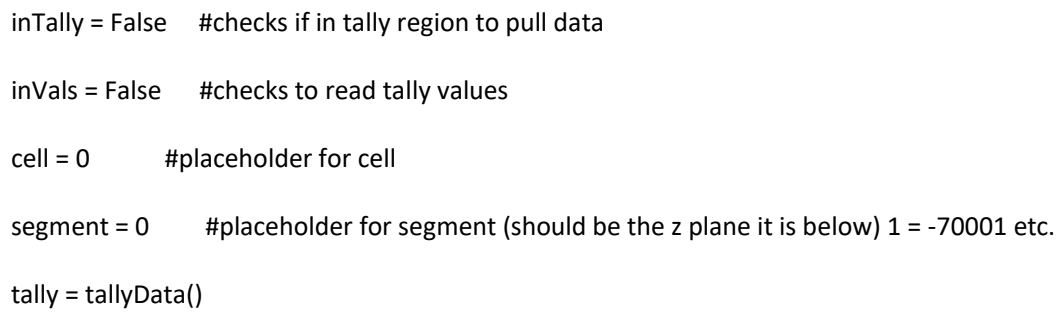




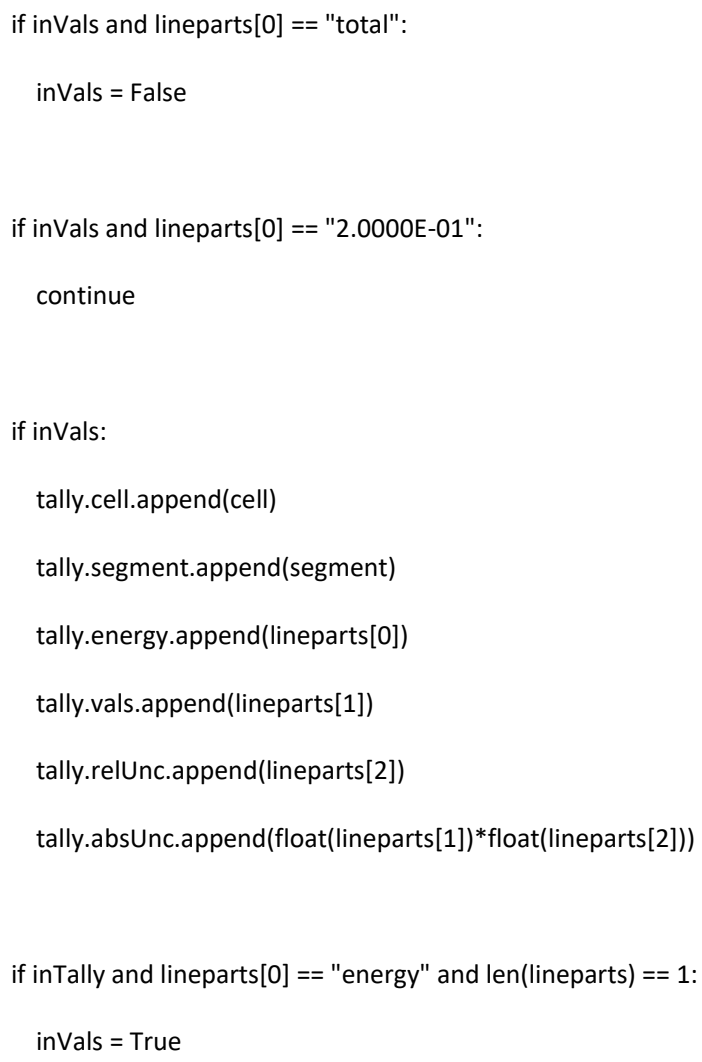




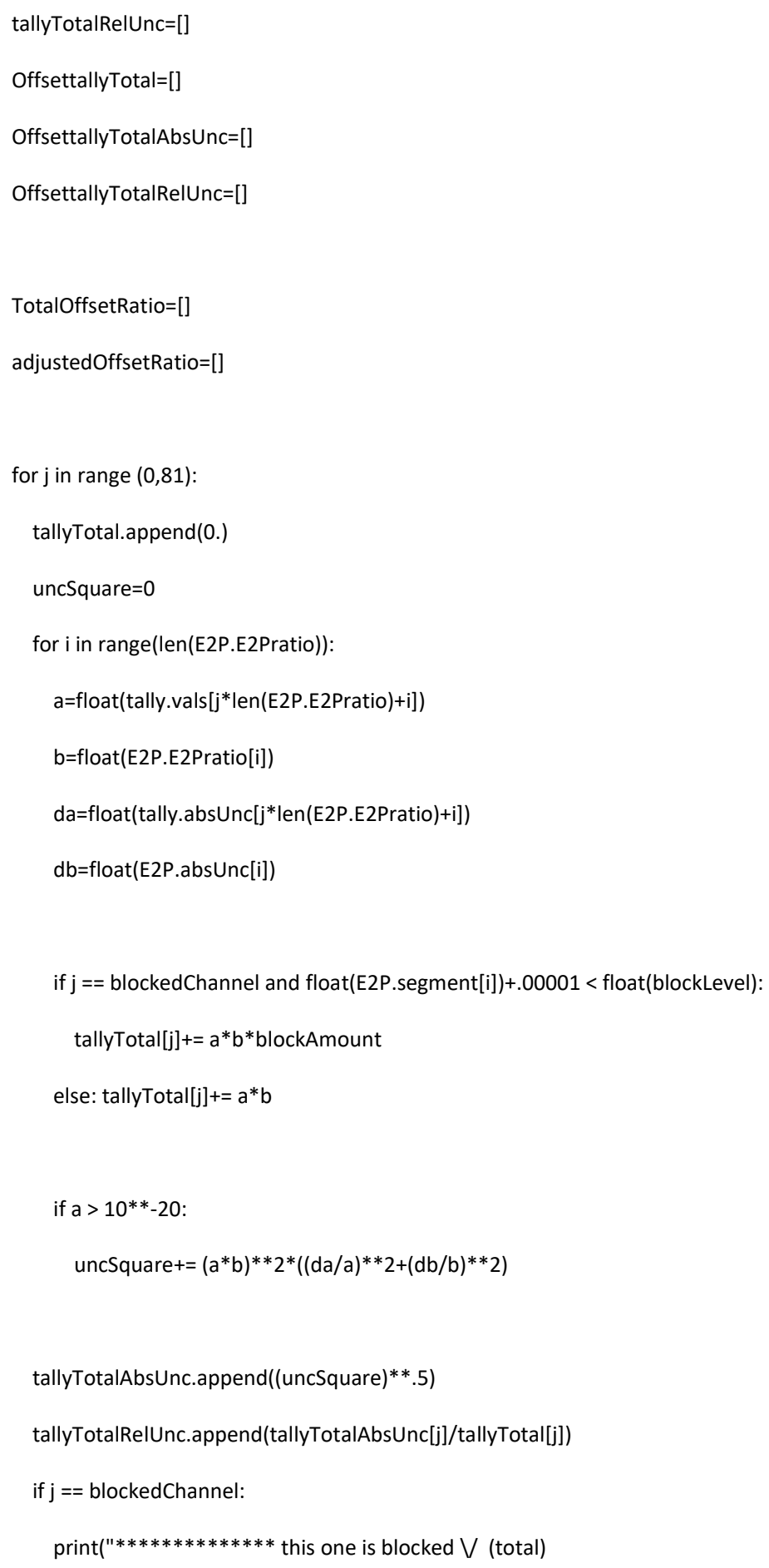




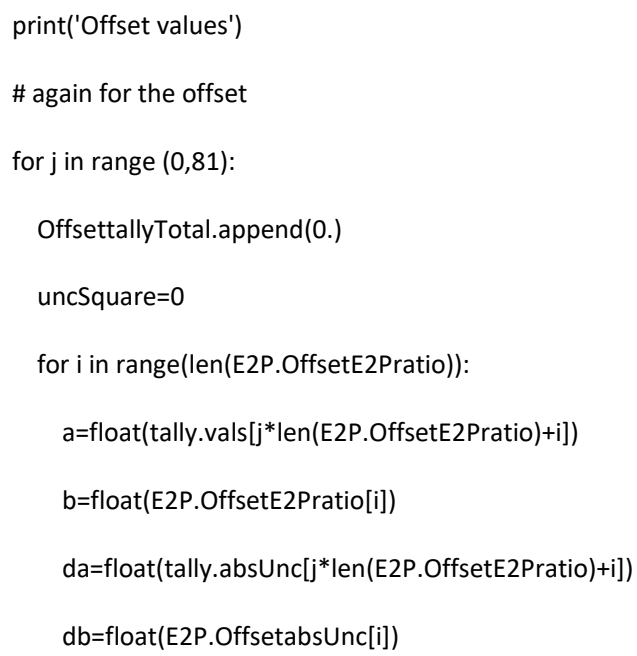




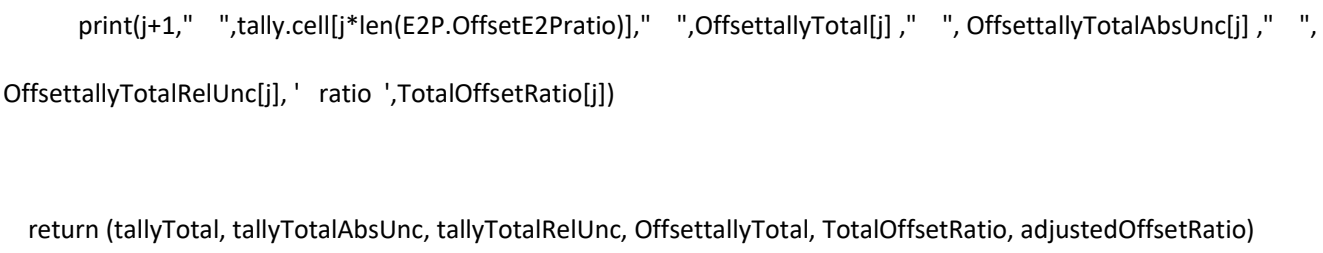




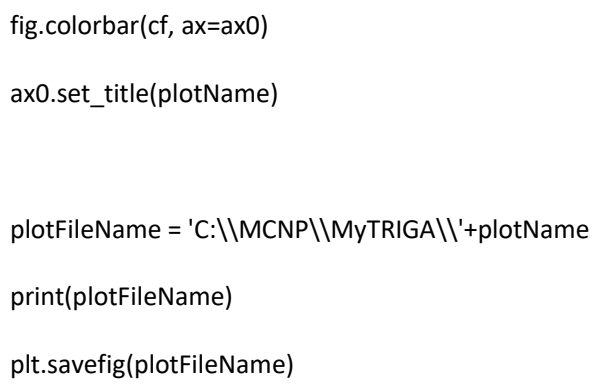


\# $\quad$ block level is the segment below which it is blocked, no mid-segemnt blocks, from 1 to 16

\# block amount is portion of channel and light blocked, 0 is full block, 1 is no block

Tally1blockLevel= 0

Tally1blockAmount $=0$

Tally2blockLevel=11

Tally2blockAmount $=.65$

\# $\quad$ end input modifying here

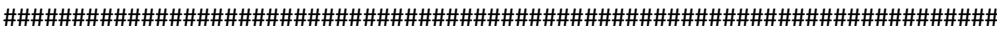

Tally1blockedChannel=yBlock1*9 +xBlock1 - 10

Tally2blockedChannel=yBlock2*9 + xBlock2 - 10

E2PFile = open("C: \\MCNP\\MyTRIGA \\EfluxToPflux.txt",'r')

E2P $=($ EfluxToPflux(E2PFile $))$

tally=(extractTallies(file1))

tally2=(extractTallies(file2))

print("\n",file1, , \n")

(tallyTotal, tallyTotalAbsUnc, tallyTotalRelUnc, OffsettallyTotal, TotalOffsetRatio, adjustedOffsetRatio) = processTallies(tally,Tally1blockedChannel,Tally1blockLevel,Tally1blockAmount)

print("\n",file2, ,'\n")

(tally2Total, tally2TotalAbsUnc, tally2TotalRelUnc, OffsettallyTotal2, TotalOffsetRatio2, adjustedOffsetRatio2) = processTallies(tally2,Tally2blockedChannel,Tally2blockLevel,Tally2blockAmount) 


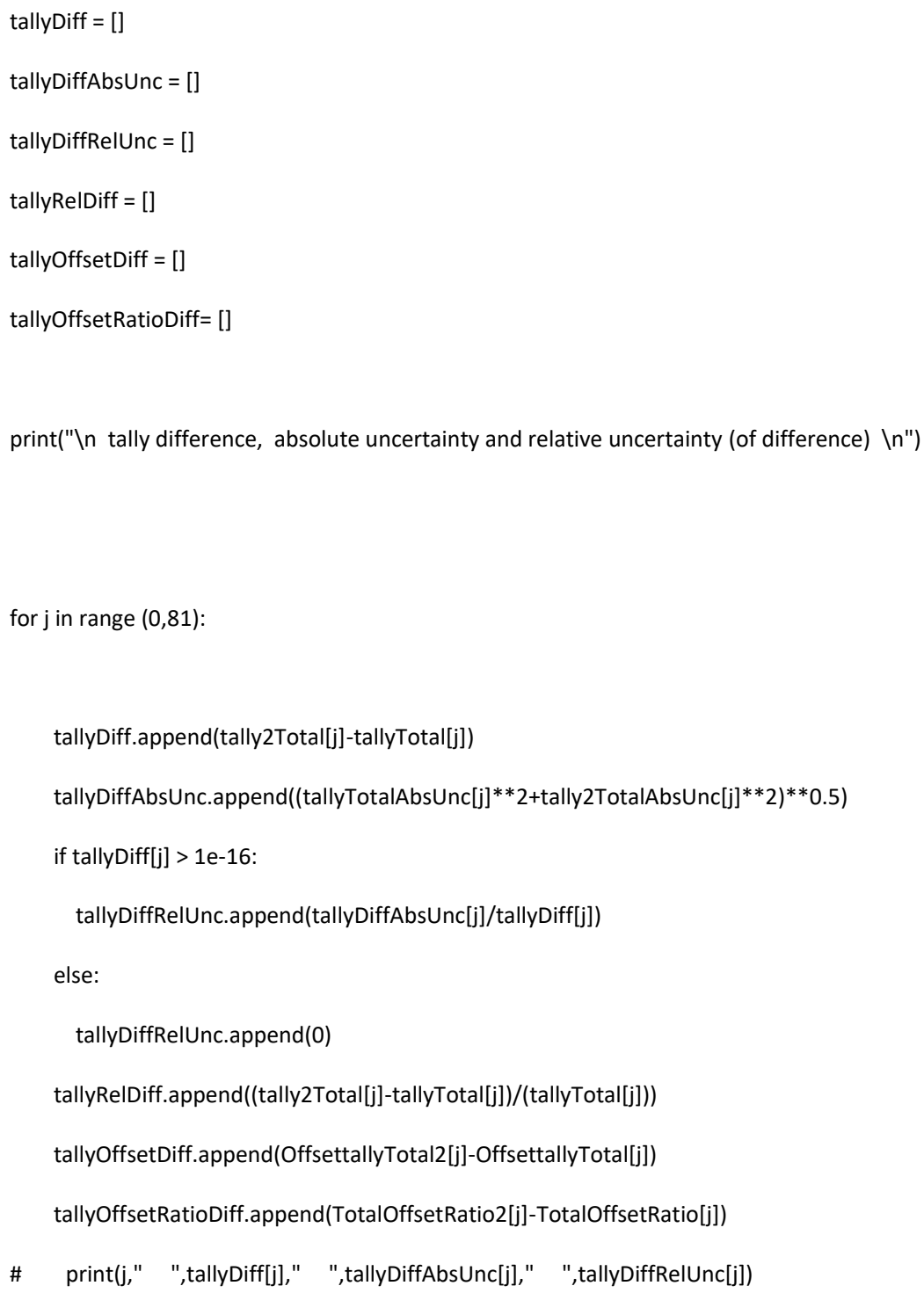




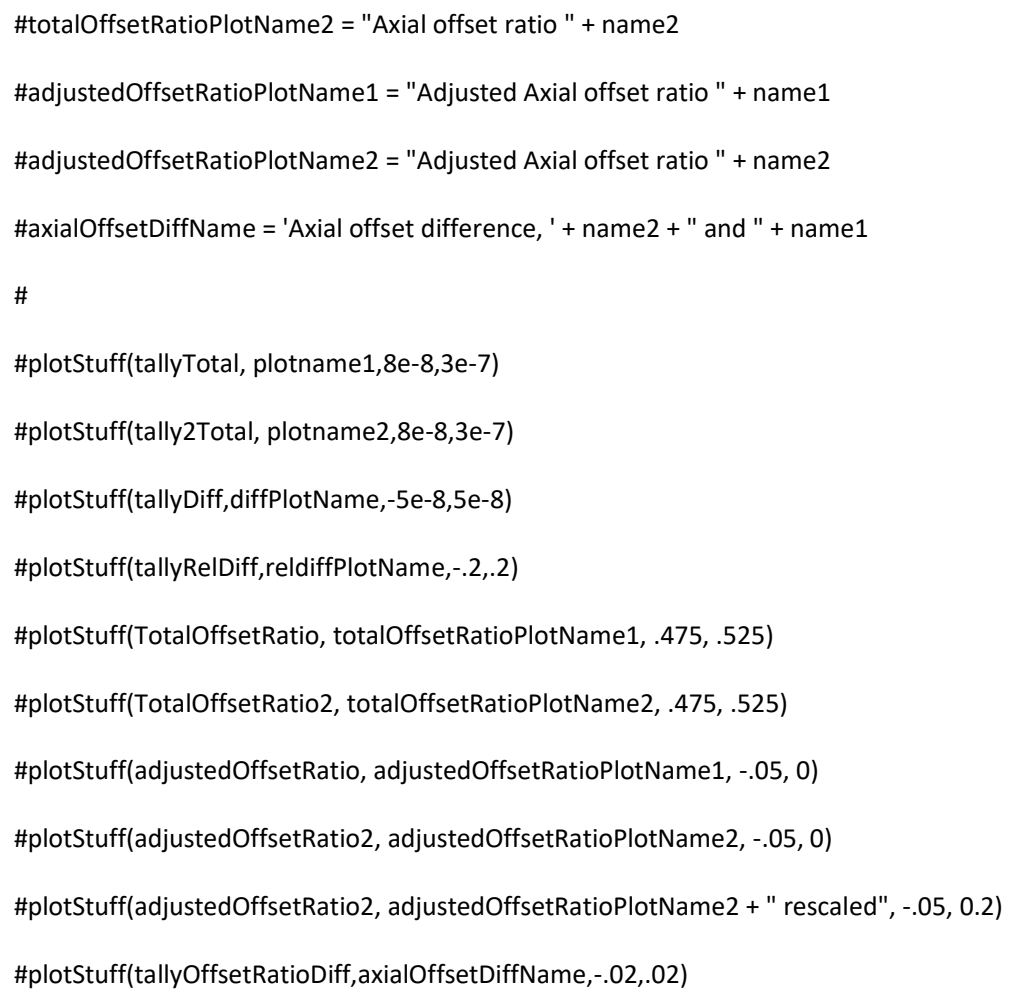

End third script

Versions of the preceding python scripts and the code MCNP are what is used for the majority of the computational and plot producing work done in this dissertation. 